\title{
Manifestly Gauge-Invariant General Relativistic Perturbation Theory: II. FRW Background and First Order
}

\author{
K. Giesel ${ }^{1 *}$, S. Hofmann ${ }^{2,3 \dagger} ;$ T. Thiemann ${ }^{1,2 \ddagger}$, O. Winkler ${ }^{2 \S}$ \\ ${ }^{1}$ MPI f. Gravitationsphysik, Albert-Einstein-Institut, \\ Am Mühlenberg 1, 14476 Potsdam, Germany \\ 2 Perimeter Institute for Theoretical Physics, \\ 31 Caroline Street N, Waterloo, ON N2L 2Y5, Canada \\ 3 NORDITA, \\ Roslagstullsbacken 23, SE-10691 Stockholm, Sweden
}

Preprint AEI-2007-151

\begin{abstract}
In our companion paper we identified a complete set of manifestly gauge-invariant observables for general relativity. This was possible by coupling the system of gravity and matter to pressureless dust which plays the role of a dynamically coupled observer. The evolution of those observables is governed by a physical Hamiltonian and we derived the corresponding equations of motion. Linear perturbation theory of those equations of motion around a general exact solution in terms of manifestly gauge invariant perturbations was then developed.

In this paper we specialise our previous results to an FRW background which is also a solution of our modified equations of motion. We then compare the resulting equations with those derived in standard cosmological perturbation theory (SCPT). We exhibit the precise relation between our manifestly gauge-invariant perturbations and the linearly gauge-invariant variables in SCPT. We find that our equations of motion can be cast into SCPT form plus corrections. These corrections are the trace that the dust leaves on the system in terms of a conserved energy momentum current density. It turns out that these corrections decay, in fact, in the late universe they are negligible whatever the value of the conserved current.

We conclude that the addition of dust which serves as a test observer medium, while implying modifications of Einstein's equations without dust, leads to acceptable agreement with known results, while having the advantage that one now talks about manifestly gauge-invariant, that is measurable, quantities, which can be used even in perturbation theory at higher orders.
\end{abstract}

\footnotetext{
*gieskri@aei.mpg.de

${ }^{\dagger}$ stefan@nordita.org

${ }^{\ddagger}$ thiemann@aei.mpg.de,tthiemann@perimeterinstitute.ca

$\S$ owinkler@perimeterinstitute.ca
} 


\section{Contents}

1 Introduction $\quad\left[\begin{array}{l}3 \\ \hline\end{array}\right.$

2 Specialisation of Linear Perturbation Theory to FRW Background 9

2.1 Gauge Invariant FRW Equations . . . . . . . . . . . . . . . . . . . . . . . 9

2.2 Specialisation of the Perturbed Equations of Motion to an FRW Background . . . . . . . 11

3 Comparison with SCPT $\quad 13$

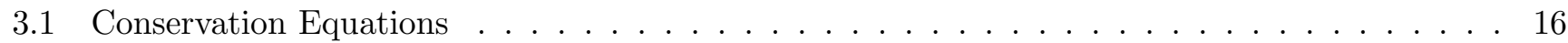

3.1.1 Momentum conservation equation . . . . . . . . . . . . . . . . . 17

3.1.2 Energy conservation equation . . . . . . . . . . . . . . . . . . 20

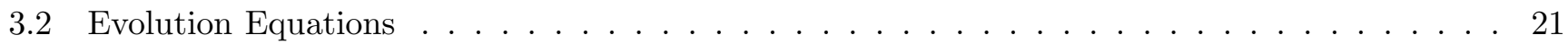

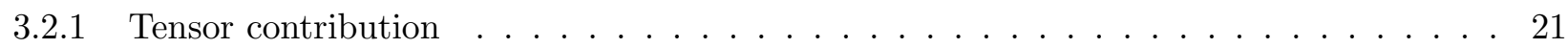

3.2 .2 Vector contribution . . . . . . . . . . . . . . . . . . 22

3.2 .3 Scalar contribution . . . . . . . . . . . . . . . . . . 22

4 Comparison with SCPT Coupled to Dust 24

4.1 Review of the SCPT Strategy for "Gravity + Scalar Field" . . . . . . . . . . . . . . . . . 25

4.2 Analysis for "Gravity + Scalar field + Dust" in Analogy with SCPT . . . . . . . . . . . . . . 26

4.3 Comparison between SCPT + Dust and our Relational Framework . . . . . . . . . . . . . . 29

4.4 Subtleties with Lapse and Shift Functions . . . . . . . . . . . . . . . . . . 31

4.5 Comparison with the SCPT framework for Lapse and Shift . . . . . . . . . . . . . . 35

5 Summary and Conclusions $\quad 36$

A Review of Standard Cosmological Perturbation Theory

A.1 Curvature and Energy Momentum Tensor . . . . . . . . . . . . . . . . . . 37

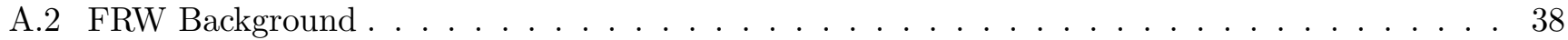

A.3 Linear Perturbations . . . . . . . . . . . . . . . . . . . . . . . . 39

A.4 Parametrisation of perturbations and linear invariants . . . . . . . . . . . 41

A.5 Tensor perturbations . . . . . . . . . . . . . . . . . . . . . 43

A.6 Vector perturbations . . . . . . . . . . . . . . . . . . . . . . 44

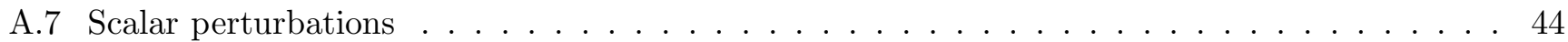

B Linear Perturbations following an Alternative Route 45

B.1 Gauge invariant FRW equations . . . . . . . . . . . . . . . . . 45

B.2 Linear perturbations of the Hamiltonian equations of motion . . . . . . . . . . . . . . 47

B.3 Second time derivative form of the perturbed equations of motion . . . . . . . . . . . . . . 49 


\section{Introduction}

In our companion paper [1] we combined the framework of relational observables [2, 3] with the Brown - Kuchař mechanism [4] in order to cast general relativity (including all known physical matter) into the form of an ordinary Hamiltonian system, with a true gauge invariant Hamiltonian which only depends on the gauge invariant geometry and matter degrees of freedom. From that foundation we then developed a manifestly gauge-invariant perturbation theory.

The motivation [5] was the trivial observation that general relativity is a gauge theory (the gauge group being the diffeomorphism group). Therefore, neither metric nor matter fields are directly observable since they are not gauge invariant and likewise Einstein's equations do not describe physical evolution of observables but rather the behaviour of non - observables under gauge transformations. On the other hand, we would like to think of Einstein's equations as describing the physical time evolution of observables. The latter point of view is often justified by the argument that the universe could be filled, in principle, by geodesic test observers who serve as a material reference system. The achievement of [4] is to implement that idea field theoretically by adding pressureless dust to the system which serves as the medium of test observers. What we did in [1] is to extend that idea further and thus to arrive at a fully gauge invariant description with a true dynamics of gauge invariant observables which directly correspond to the usual metric and matter fields.

Instead of Einstein's equations, we now have the Hamiltonian equations for those fundamental fields. The interesting question is then how close these equations come to the Einstein equations. In [1] we showed that by identifying suitable fields, one can almost exactly match our equations of motion with Einstein's. However, there are corrections which are due to the presence of the dust. More precisely, the dust itself no longer plays a role at the level of the physical observables, however it implies the existence of a conserved energy momentum current density with appropriate conservation laws which substitute the usual initial value constraints of General Relativity. This is potentially dangerous since, due to the absence of the constraints, our theory has four more physical configuration degrees of freedom than it would have without dust. Hence our theory should predict that these modes are either not excited or have decayed in order to agree with standard General Relativity (two physical degrees of freedom) plus the observationally confirmed matter of the standard model. Of course, it would be intriguing if one would find those additional modes in an experiment which then would provide indirect experimental evidence for the existence of the dust.

How much these corrections and additional degrees of freedom affect the results on the solutions of Einstein's equations is difficult to decide in full generality and analytically, therefore we will resort as usual to perturbation theory. In [1] we applied linear Hamiltonian perturbation theory to our system of Hamiltonian equations of motion. That is, one takes a general exact solution and then perturbs the equations of motion to linear order in the perturbations. Notice that in contrast to usual perturbation theory our perturbations are manifestly gauge invariant, they are full (diffeomorphism invariant) observables.

In this paper we specialise the formulae found in [1] to an FRW background which is an exact solution to our equations of motion. This allows us to study linear cosmological perturbation theory. Needless to say, this is a topic of fundamental importance in modern cosmology which goes back all the way to the pioneering work by Lifshitz [6]. Early on, it was realized that the problem of gauge freedom poses serious challenges for the use and interpretation of perturbation theory in general relativity, in general, and cosmology, in particular. A significant breakthrough was the construction of quantities that are gaugeinvariant to linear order [7]. Based on these, a satisfactory framework for linear perturbation theory for cosmology was developed and then applied by many authors. Authoritative review articles can be found in [8, 9. For the purpose of this paper, we will call the standard approach as presented in these references "standard cosmological perturbation theory" and abbreviate it as SCPT. In that context, we will always use the notation of [9]. While there seems to be little room left for significant improvements at the linear level, developing perturbation theory to higher orders is far from fully established, due to the challenge of finding gauge-invariant quantities. Some recent approaches can be found in [10, 11, 12, 13, 14. That the question of higher order perturbations is far from academic can be seen, for instance, from the growing interest in 
the issue of non-Gaussianity of cosmological perturbations [15. This is where we expect our approach to lead to major progress, due to its method of implementing gauge-invariance. Before following this line of investigation, however, we should see the results that our framework delivers at linear order and compare them with SCPT.

We find that

1. We can match our gauge invariant geometry and matter observables with the linearly gauge invariant ones of SCPT.

2. We can cast our equations of motion into SCPT form plus corrections which are proportional to the conserved energy momentum tensor current

3. The corrections decay in the late universe at least as $1 / a$ where $a$ is the physical scale factor and are thus negligible whatever their value is. This is crucial because otherwise our theory would predict, even without matter, the coexistence of vector and scalar modes apart from the gravitational waves.

We conclude that, at least in cosmological settings, our theory is in agreement with the usual results. As a special case this also extends to the Minkowski background (standard model plus gravity, in vacuum gravitational waves). Hence our theory has passed a first important consistency check. It should be mentioned here that there is an alternative approach to cosmological perturbation theory, based on the use of covariant quantities [16, 17]. We already compared it with our framework in [1], so here we will limit ourselves to a detailed comparison between our results and SCPT.

The plan of the current paper is as follows:

In section two we specialise the gauge-invariant linear perturbation theory of [1] to the FRW background. In appendix B, as a calculational check, we also follow a shorter route which is to first linearise the first time derivative equations and then to derive the second time derivative equations for the perturbations, which, as we know from appendix D of [1, must give the same result.

In section three we review the SCPT framework from a conceptual point of view. We then define a map between the linearly gauge invariant SCPT variables for gravitational perturbations and our fully gauge invariant variables. We show that under this map our equations of motion can be cast into SCPT form plus corrections which, as already stated, decay.

In section four we repeat this analysis for the case where dust is included in SCPT. We then show that the corresponding SCPT perturbation variables precisely match our gauge invariant perturbation variables when we expand them up to first order in the non gauge invariant perturbations. This demonstrates that the identification performed in section three is correct.

In section five we summarise and conclude.

In appendix A we give a concise review of SCPT from a technical point of view.

In appendix B we compute the equations derived in section two by an alternative and faster route, thus providing a consistency check. As a side result, we show that the ordinary FRW background is a solution also to our theory (with a modified energy density) and thus provides a viable background.

For the benefit of the reader, we list our notation once more on the next page. 


\section{Notation}

As a rule of thumb, gauge non invariant quantities are denoted by lower case letters, gauge invariant quantities by capital letters. The only exceptions from this rule are the dust fields $T, S^{j}, \rho, W_{j}$, their conjugate momenta $P, P_{j}, I, I^{j}$ and their associated primary constraints $Z_{j}, Z, Z^{j}$ which however disappear in the final picture. Partially gauge invariant quantities (with respect to spatial diffeomorphisms) carry a tilde. Background quantities carry a bar. Our signature convention is that of relativists, that is, mostly plus. 


\begin{tabular}{|c|c|}
\hline symbol & meaning \\
\hline$G_{N}$ & Newton constant \\
\hline$\kappa=16 \pi G_{N}$ & gravitational coupling constant \\
\hline$\lambda$ & scalar coupling constant \\
\hline$\Lambda$ & cosmological constant \\
\hline$M$ & spacetime manifold \\
\hline $\mathcal{X}$ & spatial manifold \\
\hline $\mathcal{T}$ & dust time manifold \\
\hline $\mathcal{S}$ & dust space manifold \\
\hline$\mu, \nu, \rho, . .=0, . ., 3$ & tensor indices on $M$ \\
\hline$a, b, c, . .=1,2,3$ & tensor indices on $\mathcal{X}$ \\
\hline$i, j, k, . .=1,2,3$ & tensor indices on $\mathcal{S}$ \\
\hline$X^{\mu}$ & coordinates on $M$ \\
\hline$x^{a}$ & coordinates on $\mathcal{X}$ \\
\hline$\sigma^{j}$ & coordinates on $\mathcal{S}$ \\
\hline$t$ & foliation parameter \\
\hline$\tau$ & dust time coordinate \\
\hline$Y_{t}^{\mu}$ & one parameter family of embeddings $\mathcal{S} \rightarrow M$ \\
\hline $\mathcal{S}_{t}=Y_{t}(\mathcal{S})$ & leaves of the foliation \\
\hline$g_{\mu \nu}$ & metric on $M$ \\
\hline$q_{a b}$ & (pullback) metric on $\mathcal{X}$ \\
\hline$\tilde{q}_{i j}$ & (pullback) metric on $\mathcal{S}$ \\
\hline$Q_{i j}$ & Dirac observable associated to $q_{a b}$ \\
\hline$p^{a b}$ & momentum conjugate to $q_{a b}$ \\
\hline$\tilde{p}^{i j}$ & momentum conjugate to $\tilde{q}_{i j}$ \\
\hline$P^{i j}$ & momentum conjugate to $Q_{i j}$ \\
\hline$\zeta$ & scalar field on $M$ \\
\hline$\xi$ & scalar field on $\mathcal{X}$ \\
\hline$\tilde{\xi}$ & pullback scalar field on $\mathcal{S}$ \\
\hline$\Xi$ & Dirac observable associated to $\xi$ \\
\hline$\pi$ & momentum conjugate to $\xi$ \\
\hline$\tilde{\pi}$ & momentum conjugate to $\tilde{\xi}$ \\
\hline$\Pi$ & momentum conjugate to $\Xi$ \\
\hline$v$ & potential of $\zeta, \xi, \tilde{\xi}, \Xi$ \\
\hline$T$ & dust time field on $\mathcal{X}$ \\
\hline$\tilde{T}$ & dust time field on $\mathcal{S}$ \\
\hline$S^{j}$ & dust space fields on $\mathcal{X}$ \\
\hline$\rho$ & dust energy density on $M, \mathcal{X}$ \\
\hline$W_{j}$ & dust Lagrange multiplier field on $M, \mathcal{X}$ \\
\hline$U=-d T+W_{j} d S^{j}$ & dust deformation covector field on $M$ \\
\hline$J=\operatorname{det}(\partial S / \partial x)$ & dust field spatial density on $\mathcal{X}$ \\
\hline$P$ & momentum conjugate to $T$ \\
\hline$\tilde{P}$ & momentum conjugate to $\tilde{T}$ \\
\hline$P_{j}$ & momentum conjugate to $S^{j}$ \\
\hline$I$ & momentum conjugate to $\rho$ \\
\hline$I^{j}$ & momentum conjugate to $W_{j}$ \\
\hline$Z_{j}, Z, Z^{j}$ & dust primary constraints on $\mathcal{X}$ \\
\hline$\mu^{j}, \mu, \mu_{j}$ & dust primary constraint Lagrange multipliers on $\mathcal{X}$ \\
\hline
\end{tabular}




\begin{tabular}{|c|c|}
\hline$\varphi$ & diffeomorphism of $\mathcal{X}$ \\
\hline$n^{\mu}$ & unit normal of spacelike hypersurface on $M$ \\
\hline$n$ & coordinate lapse function on $\mathcal{X}$ \\
\hline$n^{a}$ & coordinate shift function on $\mathcal{X}$ \\
\hline$p$ & momentum conjugate to $\mathrm{n}$ \\
\hline$p_{a}$ & momentum conjugate to $n^{a}$ \\
\hline$z, z_{a}$ & primary constraint for lapse, shift \\
\hline$\nu, \nu^{a}$ & lapse and shift primary constraint Lagrange multipliers \\
\hline$\phi, \psi, B, E$ & SCPT scalars on $\mathcal{X}, \mathcal{S}$ \\
\hline$S_{a}, F_{a}$ & SCPT transversal vectors on $\mathcal{X}$ \\
\hline$S_{j}, F_{j}$ & SCPT transversal vectors on $\mathcal{S}$ \\
\hline$h_{a b}$ & SCPT transverse tracefree tensor on $\mathcal{X}$ \\
\hline$h_{j k}$ & SCPT transverse tracefree tensor on $\mathcal{S}$ \\
\hline$\Phi, \Psi$ & linear gauge invariant completions of $\phi, \psi$ \\
\hline$V_{a}$ & linear gauge invariant completions of $F_{a}$ \\
\hline$V_{j}$ & linear gauge invariant completions of $F_{j}$ \\
\hline$c_{a}^{\text {tot }}$ & total spatial diffeomorphism constraint on $\mathcal{X}$ \\
\hline$c_{j}^{\mathrm{tot}}=S_{j}^{a} c_{a}^{\mathrm{tot}}$ & total spatial diffeomorphism constraint on $\mathcal{X}$ \\
\hline$c^{\text {tot }}$ & total Hamiltonian constraint on $\mathcal{X}$ \\
\hline$c_{a}$ & non - dust contribution to spatial diffeomorphism constraint on $\mathcal{X}$ \\
\hline$c_{j}=S_{j}^{a} c_{a}$ & non - dust contribution to spatial diffeomorphism constraint on $\mathcal{X}$ \\
\hline$\tilde{c}_{j}$ & non - dust contribution to spatial diffeomorphism constraint on $\mathcal{S}$ \\
\hline $\begin{array}{l}C_{j} \neq \tilde{c}_{j} \\
\quad c\end{array}$ & $\begin{array}{l}\text { momentum density: Dirac observable associated to } \tilde{c}_{j} \\
\text { non - dust contribution to Hamiltonian constraint on } \mathcal{X}\end{array}$ \\
\hline$\tilde{c}$ & non - dust contribution to Hamiltonian constraint on $\mathcal{S}$ \\
\hline$C \neq \tilde{c}$ & Dirac observable associated to $\tilde{c}$ \\
\hline$h$ & energy density on $\mathcal{X}$ \\
\hline$\tilde{h}$ & energy density on $\mathcal{S}$ \\
\hline$H=\tilde{h}$ & energy density: Dirac observable associated to $\tilde{h}$ \\
\hline$h_{j}=c_{j}^{\mathrm{tot}}-P_{j}$ & auxiliary density on $\mathcal{X}$ \\
\hline$\epsilon$ & numerical energy density on $\mathcal{S}$ \\
\hline$\epsilon_{j}$ & numerical momentum density on $\mathcal{S}$ \\
\hline $\mathbf{H}=\int_{\mathcal{S}} d^{3} \sigma H$ & physical Hamiltonian, energy \\
\hline$L$ & Lagrange density associated to $H$ \\
\hline $\mathbf{L}=\int_{\mathcal{S}} d^{3} \sigma L$ & physical Lagrangian \\
\hline$V_{j k}$ & velocity associated to $Q_{j k}$ \\
\hline$\Upsilon$ & velocity associated to $\Xi$ \\
\hline$N=C / H$ & dynamical lapse function on $\mathcal{S}$ \\
\hline$N_{j}=-C_{j} / H$ & dynamical shift function on $\mathcal{S}$ \\
\hline$N^{j}=Q^{j k} N_{k}$ & dynamical shift function on $\mathcal{S}$ \\
\hline$\nabla_{\mu}$ & $g_{\mu \nu}$ compatible covariant differential on $M$ \\
\hline$D_{a}$ & $q_{a b}$ compatible covariant differential on $\mathcal{X}$ \\
\hline$\tilde{D}_{j}$ & $\tilde{q}_{j k}$ compatible covariant differential on $\mathcal{S}$ \\
\hline $\bar{Q}_{j k}$ & $\begin{array}{l}Q_{j k} \text { compatible covariant differential on } \mathcal{S} \\
\text { background spatial metric }\end{array}$ \\
\hline $\bar{P}^{j k}$ & background momentum conjugate to $\bar{Q}_{j k}$ \\
\hline $\bar{\Xi}$ & background scalar field \\
\hline $\bar{\Pi}$ & background momentum conjugate to $\bar{\Xi}$ \\
\hline $\bar{\nu}=\frac{1}{2 \lambda}\left[\dot{\bar{\Xi}}^{2}+v(\bar{\Xi})\right]$ & background scalar energy density \\
\hline $\bar{p}=\frac{1}{2 \lambda}\left[\dot{\bar{\Xi}}^{2}-v(\bar{\Xi})\right]$ & background scalar pressure \\
\hline
\end{tabular}


$G_{j k m n}=Q_{j(m} Q_{n) k}-\frac{1}{2} Q_{j k} Q_{m n} \quad$ physical DeWitt bimetric

$\left[G^{-1}\right]^{j k m n}=Q^{j(m} Q^{n) k}-Q^{j k} Q^{m n} \quad$ inverse physical DeWitt bimetric

$\bar{G}_{j k m n}=\delta_{j(m} \delta_{n) k}-\frac{1}{2} \delta_{j k} \delta_{m n} \quad$ flat background DeWitt bimetric

$\left[\bar{G}^{-1}\right]^{j k m n}=\delta^{j(m} \delta^{n) k}-\delta^{j k} \delta^{m n} \quad$ inverse flat background DeWitt bimetric 


\section{Specialisation of Linear Perturbation Theory to FRW Background}

In this section we want to specialise the perturbed equations that were derived in our companion paper [1] for an arbitrary background to the case of an FRW spacetime. The perturbed matter scalar field $\delta \Xi$, as well as the perturbed three metric $\delta Q_{j k}$ are manifestly gauge invariant. This gauge invariance is not restricted to linear perturbations only, but extends to arbitrarily high orders. The underlying reason is the fact that we applied perturbation theory to an already manifestly gauge invariant physical system, since the quantities $Q_{j k}$ and $\Xi$ are gauge invariant by construction. Hence, any function of $\delta \Xi, \delta Q_{j k}$ is still a gauge invariant quantity. For this reason higher powers of the perturbation that occur in higher order perturbation theory will in our framework not destroy gauge invariance. Therefore, in principle, it is possible to analyse cosmological perturbation theory also in arbitrarily high orders. However, in this section we will focus on cosmological perturbation theory up to linear order, the discussion on higher order equations will be left for future work.

Due to the homogeneity and isotropy associated with FRW, the final second order equations of motion, shown in equation (6.13) in [1] for the linear perturbation of the matter scalar field $\delta \Xi$, and in equation (6.31) for the perturbed three metric $\delta Q_{j k}$, simplify drastically. A similar simplification occurs for the coefficient functions that were introduced in section 6.3 in [1] in order to write those second order equations in a less complicated form. Before actually discussing the specialisation to FRW for the perturbed equations of motions, we will first consider the unperturbed second order time derivative equations of motion for $\Xi$ and $Q_{j k}$, derived in [1] in equation (4.24) and (4.25), respectively, and check that they yield a gauge invariant version of the ordinary FRW equation of standard cosmology. An alternative derivation of the gauge invariant FRW equation can be found in appendix B in section B.1, where already the first order time derivative Hamiltonian equations are specialised to FRW before the second order time derivative equations are calculated.

\subsection{Gauge Invariant FRW Equations}

In the remaining part of this paper we will indicate quantities which are specialised to FRW spacetimes with a bar. Due to the homogeneity of an FRW spacetime, all spatial derivatives vanish. For this reason the matter as well as the geometry part of the (gauge invariant) diffeomorphism constraint, denoted by $\bar{C}_{j}^{\text {matter }}$ and $\bar{C}_{j}^{\text {geo }}$, vanish as can be seen from equation (2.1):

$$
\begin{aligned}
C_{j}(\sigma)= & {\left[-2 \bar{Q}_{j k}\left(\bar{D}_{k} \bar{P}^{k l}\right)+\bar{\Pi}_{, j}\right](\tau, \sigma) } \\
=: & \bar{C}_{j}^{\text {geo }}(\tau, \sigma)+\bar{C}_{j}^{\text {matter }}(\tau, \sigma) \\
\bar{C}(\tau, \sigma)= & \frac{1}{\kappa}\left[\frac{1}{\sqrt{\operatorname{det} \bar{Q}}}\left[\bar{Q}_{j m} \bar{Q}_{k n}-\frac{1}{2} \bar{Q}_{j k} \bar{Q}_{m n}\right] \bar{P}^{j k} \bar{P}^{m n}-\sqrt{\operatorname{det} \bar{Q}} \bar{R}^{(3)}[\bar{Q}]+2 \Lambda \sqrt{\operatorname{det} \bar{Q}}\right](\tau, \sigma) \\
& +\frac{1}{2 \lambda}\left[\frac{\bar{\Pi}^{2}}{\sqrt{\operatorname{det} \bar{Q}}}+\sqrt{\operatorname{det} \bar{Q}}\left[\bar{Q}^{j k}\left(\bar{D}_{j} \Xi\right)\left(\bar{D}_{k} \bar{\Xi}\right)+v(\bar{\Xi})\right]\right](\tau, \sigma) \\
=: & \bar{C}^{\text {geo }}(\tau, \sigma)+\bar{C}^{\text {matter }}(\tau, \sigma),
\end{aligned}
$$

where $\bar{D}_{j}$ is the covariant differential compatible with $\bar{Q}_{j k}$ and we used that the Christoffel symbols with spatial indices vanish in the case of FRW. The results in [1] showed that one of the effects when dust is used as a clock is that we obtain a phase space dependent and thus dynamical lapse function and shift vector, which we denoted by $\bar{N}$ and $\bar{N}_{j}$, respectively. Their explicit form is given by

$$
\bar{N}=\frac{\bar{C}(\tau, \sigma)}{\bar{H}(\sigma)} \quad \text { and } \quad \bar{N}_{j}=-\frac{\bar{C}_{j}(\tau, \sigma)}{\bar{H}(\sigma)}
$$


where

$$
\bar{H}(\sigma)=\sqrt{\bar{C}(\tau, \sigma)^{2}-\bar{Q}^{j k}(\tau, \sigma) \bar{C}_{j}(\sigma) C_{k}(\sigma)}
$$

is the physical Hamiltonian density. This is the density of the physical Hamiltonian $\overline{\mathbf{H}}$ that is generating the (dust) time evolution for all observables, in particular for $\bar{\Xi}$ and $\bar{Q}_{j k}$. Knowing now that $\bar{C}_{j}$ vanishes for FRW, we get immediately that the shift vector vanishes, too. Hence in the case of FRW, all terms proportional to $\bar{N}_{j}$ can be omitted in equations (4.17) and (4.18). Furthermore from equation (2.3) and the definition of $\bar{N}$ and $\bar{N}_{j}$ we get for FRW

$$
\bar{N}=\frac{\bar{C}}{\bar{H}}=\frac{\sqrt{\bar{H}^{2}+\bar{Q}^{j k} \bar{C}_{j} \bar{C}_{k}}}{\bar{H}}=\sqrt{1+\bar{Q}^{j k} \bar{N}_{j} \bar{N}_{k}}=1 .
$$

We observe that $\bar{N}$ and $\bar{N}_{j}$ are not independent quantities and that for the special case of FRW the lapse function takes the constant value +1 . Keeping this in mind and taking into account that all terms containing spatial derivatives vanish, the equations (4.17) and (4.18) for $\ddot{\Xi}$ and $\ddot{Q}_{j k}$ in our companion paper [1] reduce to

$$
\begin{aligned}
\ddot{\bar{\Xi}} & =-\frac{(\sqrt{\operatorname{det} \bar{Q}})}{\sqrt{\operatorname{det} \bar{Q}}} \dot{\bar{\Xi}}-\frac{1}{2} v^{\prime}(\bar{\Xi}) \\
\ddot{\bar{Q}}_{j k} & =-\frac{(\sqrt{\operatorname{det} \bar{Q}})}{\sqrt{\operatorname{det} \bar{Q}}} \dot{\bar{Q}}_{j k}+\bar{Q}^{m n} \dot{\bar{Q}}_{m j} \dot{\bar{Q}}_{n k}+\bar{Q}_{j k}\left(-\frac{\kappa}{2 \sqrt{\operatorname{det} \bar{Q}}} \bar{C}+2 \Lambda+\frac{\kappa}{2 \lambda} v(\bar{\Xi})\right) .
\end{aligned}
$$

Using that $\bar{Q}_{j k}=A^{2} \delta_{j k}$ where $A$ is the physical scale factor, that is the gauge invariant extension of the usual non gauge invariant scale factor $a$ used in standard cosmology, we obtain for $\ddot{\bar{\Xi}}$

$$
\ddot{\bar{\Xi}}=-3\left(\frac{\dot{A}}{A}\right) \dot{\bar{\Xi}}-\frac{1}{2} v^{\prime}(\bar{\Xi})
$$

This agrees (formally) with the usual FRW equation for a scalar field. The agreement is formal in the sense that here we have an evolution equation for a gauge-invariant and thus physical scalar field $\bar{\Xi}$. Also the equation contains the physical scale factor $A$, not $a$ which usually occurs in this equation. Furthermore the dot refers to a derivative with respect to dust time $\tau$ in the equation above. For $\ddot{\bar{Q}}_{j k}$ we need the explicit form of the matter, as well as the geometry part of the Hamiltonian constraint, whose sum is denoted by $\bar{C}$. Specialised to FRW it is given by

$$
\bar{C}=A^{3}\left[\frac{1}{\kappa}\left(2 \Lambda-6\left(\frac{\dot{A}}{A}\right)^{2}\right)+\frac{1}{2 \lambda}\left(\dot{\bar{\Xi}}^{2}+v(\bar{\Xi})\right)\right] .
$$

Inserting this back into equation (2.5) and performing the derivatives yields

$$
\left(\frac{\ddot{A}}{A}\right)=-\frac{1}{2}\left(\frac{\dot{A}}{A}\right)^{2}+\frac{1}{2} \Lambda-\frac{\kappa}{4 \lambda} \frac{1}{2}\left(\dot{\Xi}^{2}-v(\bar{\Xi})\right)
$$

As shown in our companion paper [1], the Hamiltonian density $\bar{H}$ in equation (2.3) is a constant of motion. In the case of FRW, $\bar{H}$ reduces to

$$
\bar{H}=\sqrt{\bar{C}^{2}-\bar{Q}^{j k} \bar{N}_{j} \bar{N}_{k}}=\sqrt{\bar{C}^{2}}=\bar{C}
$$

due to the vanishing of the shift vector. Denoting the energy density by $\epsilon$, we have the conservation law

$$
\bar{H}=\bar{C}=A^{3}\left[\frac{1}{\kappa}\left(2 \Lambda-6\left(\frac{\dot{A}}{A}\right)^{2}\right)+\frac{1}{2 \lambda}\left(\dot{\bar{\Xi}}^{2}+v(\bar{\Xi})\right)\right]=\epsilon .
$$


Note that $\bar{C}$ is not a constraint here, since only the total sum $\bar{C}^{\text {tot }}=\bar{C}^{\text {geo }}+\bar{C}^{\text {matter }}+\bar{C}^{\text {dust }}$, with the dust included, is constrained to vanish. The conservation law above can be equivalently written as

$$
3\left(\frac{\dot{A}}{A}\right)^{2}=-\frac{\epsilon \kappa}{2 A^{3}}+\Lambda+\frac{\kappa}{4 \lambda}\left(\dot{\bar{\Xi}}^{2}+v(\bar{\Xi})\right) .
$$

Replacing $(\dot{A} / A)^{2}$ in equation (2.8) by the expression in the equation above, we end up with

$$
3\left(\frac{\ddot{A}}{A}\right)=\Lambda-\frac{\kappa}{4}\left[\frac{1}{2 \lambda}\left(\dot{\bar{\Xi}}^{2}+v(\bar{\Xi})\right)+3 \frac{1}{2 \lambda}\left(\dot{\bar{\Xi}}^{2}-v(\bar{\Xi})\right)-\frac{\epsilon}{A^{3}}\right] .
$$

Introducing the matter density and pressure as well as the dust density

$$
\begin{aligned}
\bar{\rho}^{\text {matter }} & =\frac{\bar{C}^{\text {matter }}}{A^{3}}=\frac{1}{2 \lambda}\left(\dot{\bar{\Xi}}^{2}+v(\bar{\Xi})\right) \\
\bar{\rho}^{\text {dust }} & =\frac{\bar{C}^{\text {dust }}}{A^{3}}=-\frac{\bar{C}^{\text {matter }}+\bar{C}^{\text {matter }}}{A^{3}}-\frac{\bar{C}}{A^{3}}=-\frac{\epsilon}{A^{3}} \\
\bar{p}^{\text {matter }} & \left.=\frac{1}{2 \lambda}{\left(\dot{\bar{\Xi}}^{2}\right.}^{2}-v(\bar{\Xi})\right),
\end{aligned}
$$

we can rewrite equation (2.12) as

$$
3\left(\frac{\ddot{A}}{A}\right)=\Lambda-\frac{\kappa}{4}\left(\bar{\rho}^{\text {matter }}+\bar{\rho}^{\text {dust }}+3 \bar{p}^{\text {matter }}\right) .
$$

This again coincides formally with the usual FRW equation for the gravitational part if the system "gravity + scalar field + dust" is considered and the dust is assumed to be pressureless as is the case in our framework. The minus sign in $\bar{\rho}^{\text {dust }}$ in equation (2.13) reflects the phantom nature of the dust which was discussed in detail in section 2.3.4 of our companion paper [1].

Summarising, when (phantom) dust is employed in order to deparametrise the constraints of General Relativity, the resulting physical Hamiltonian generates equations of motion that formally agree with the standard FRW equations. For this reason, the dust clock seems to be the clock closest to the framework of standard cosmology where the Hamiltonian constraint is taken as a true Hamiltonian. In the next section we will move on to the discussion of the perturbed equations of motion for $\delta \Xi$ and $\delta Q_{j k}$.

\subsection{Specialisation of the Perturbed Equations of Motion to an FRW Background}

Let us now specialise the perturbed equations for $\delta \ddot{\Xi}$ and $\delta \ddot{Q}_{j k}$, derived in our companion paper [1], to the case of an FRW background. As discussed in the last section, in this case the lapse function takes the constant value $\bar{N}=1$ and the shift vector $\bar{N}_{j}$ vanishes. Taking into account that all spatial derivatives of background quantities (these are indicated with a bar) vanish, the coefficient functions for $\delta \Xi$ that can be found in equation (6.33), (6.34) and (6.35) of [1] simplify to

$$
\begin{aligned}
{\left[C_{\Xi}\right] } & =\frac{\partial^{2}}{\partial \tau^{2}}+\frac{(\sqrt{\operatorname{det} \bar{Q}})}{\sqrt{\operatorname{det} \bar{Q}}} \frac{\partial}{\partial \tau}-\Delta+\frac{1}{2} v^{\prime \prime}(\bar{\Xi})=\frac{\partial^{2}}{\partial \tau^{2}}+3\left(\frac{\dot{A}}{A}\right) \frac{\partial}{\partial \tau}-\frac{1}{A^{2}} \delta^{j k} \frac{\partial}{\partial x^{j}} \frac{\partial}{\partial x^{k}}+\frac{1}{2} v^{\prime \prime}(\bar{\Xi}) \\
{\left[C_{\Xi}\right]^{j k} } & =-\frac{1}{2} \dot{\Xi} \frac{\partial}{\partial \tau}\left(\bar{Q}^{j k}\right)=-\frac{1}{2} \dot{\bar{\Xi}}^{j k}-\frac{1}{2} \dot{\Xi} \bar{Q}^{j k} \frac{\partial}{\partial \tau}=+\frac{1}{A^{2}}\left(\frac{\dot{A}}{A}\right) \dot{\Xi} \delta^{j k}-\frac{1}{2} \frac{1}{A^{2}} \dot{\bar{\Xi}} \delta^{j k} \frac{\partial}{\partial \tau} \\
{\left[C_{\Xi}\right]^{j} } & =\dot{\Xi} \bar{Q}^{j k} \frac{\partial}{\partial x^{k}}=\frac{1}{A^{2}} \dot{\bar{\Xi}} \delta^{j k} \frac{\partial}{\partial x^{k}} .
\end{aligned}
$$

Using these coefficients, we obtain the following perturbed equation

$\left[\frac{\partial^{2}}{\partial \tau^{2}}+3\left(\frac{\dot{A}}{A}\right) \frac{\partial}{\partial \tau}-\frac{1}{A^{2}} \delta^{j k} \frac{\partial}{\partial x^{j}} \frac{\partial}{\partial x^{k}}+\frac{1}{2} v^{\prime \prime}(\bar{\Xi})\right] \delta \Xi=\left[+\frac{1}{A^{2}}\left(\frac{\dot{A}}{A}\right) \dot{\bar{\Xi}} \delta^{j k}-\frac{1}{2} \frac{1}{A^{2}} \dot{\bar{\Xi}} \delta^{j k} \frac{\partial}{\partial \tau}\right] \delta Q_{j k}+\left[\frac{1}{2} \frac{1}{A^{2}} \dot{\bar{\Xi}} \delta^{j k}\right] \delta N_{j}$. 
When performing the derivatives and rearranging the terms, the equation above can be rewritten as

$$
\delta \ddot{\Xi}=-3\left(\frac{\dot{A}}{A}\right) \delta \dot{\Xi}+\frac{1}{A^{2}} \delta^{j k} \delta \Xi_{, j k}-\frac{1}{2} v^{\prime \prime}(\bar{\Xi}) \delta \Xi+\frac{1}{A^{2}}\left(\frac{\dot{A}}{A}\right) \dot{\Xi} \delta Q_{j j}-\frac{1}{2} \frac{1}{A^{2}} \dot{\bar{\Xi}} \delta \dot{Q}_{j j}+\frac{1}{A^{2}} \dot{\bar{\Xi}} \delta N_{j, j} .
$$

This equation agrees with equation $(\overline{B .22})$ in appendix $B$ where an alternative route is taken to obtain the second order equation of motion. There we first perturb the Hamiltonian first order equation and derived then the second order perturbed equations. For linear perturbation theory it is proven in appendix D of [1] that these two ways of deriving the second order equation of motion are equivalent. Compared to the perturbed equations in SCPT we obtain an additional term, namely the last term on the right-hand side of equation (2.17). This term arises from the interaction of the clock, namely the dust, with the physical system. However, in the next section where we compare our results in detail with the results from SCPT, we will show that the physical predictions of both frameworks are in agreement.

Let us now consider the coefficients for $\delta Q_{j k}$ in equation (6.37)-(6.41) of [1] and their specialisation to FRW. Taking again into account that $N=1$, that all terms proportional to $N_{j}$ can be omitted and that all spatial derivatives of background quantities are zero, we obtain

$$
\begin{aligned}
& {\left[C_{Q}\right]=\frac{\partial^{2}}{\partial \tau^{2}}+\frac{\kappa}{2 \sqrt{\operatorname{det} \bar{Q}}} \bar{C}-\left(2 \Lambda+\frac{\kappa}{2 \lambda} v(\Xi)\right)-\bar{D}_{m} \bar{D}_{n} \bar{Q}^{m n}+\frac{(\sqrt{\operatorname{det} \bar{Q}})}{\sqrt{\operatorname{det} \bar{Q}}} \frac{\partial}{\partial \tau}} \\
& =\frac{\partial^{2}}{\partial \tau^{2}}-3\left(\frac{\dot{A}}{A}\right)^{2}-\Lambda+\frac{\kappa}{4 \lambda}\left(\dot{\bar{\Xi}}^{2}-v(\bar{\Xi})\right)-\bar{D}_{m} \bar{D}_{n} \bar{Q}^{m n}+3\left(\frac{\dot{A}}{A}\right) \frac{\partial}{\partial \tau} \\
& {\left[A_{Q}\right]_{j k}=-\bar{Q}_{j k} \frac{\kappa}{2 \lambda} \dot{\bar{\Xi}} \frac{\partial}{\partial \tau}+\bar{Q}_{j k} \frac{\kappa}{4 \lambda} v^{\prime}(\bar{\Xi})=\left[-\frac{1}{A^{2}} \frac{\kappa}{2 \lambda} \dot{\bar{\Xi}} \frac{\partial}{\partial \tau}+\frac{1}{A^{2}} \frac{\kappa}{4 \lambda} v^{\prime}(\bar{\Xi})\right] \delta_{j k}} \\
& {\left[B_{Q}\right]_{j k}=0} \\
& {\left[C_{Q}\right]_{(k}^{m}=2 \bar{Q}^{m n} \dot{\bar{Q}}_{n(k} \frac{\partial}{\partial \tau}-2 \bar{D}_{n} \bar{D}_{(k} \bar{Q}^{m n}=4\left(\frac{\dot{A}}{A}\right) \delta_{(k}^{m} \frac{\partial}{\partial \tau}-2 \bar{D}_{n} \bar{D}_{(k} \bar{Q}^{m n}} \\
& {\left[C_{Q}\right]_{j k}^{m}=\dot{\bar{Q}}_{j k} \frac{\partial}{\partial x^{n}} \bar{Q}^{m n}+\frac{(\sqrt{\operatorname{det} \bar{Q}})}{\sqrt{\operatorname{det} \bar{Q}}}\left(\bar{Q}_{n k} \frac{\partial}{\partial x^{j}} \bar{Q}^{m n}+\bar{Q}_{m j} \frac{\partial}{\partial x^{k}} \bar{Q}^{m n}\right)+\frac{1}{2} \bar{Q}_{j k} \dot{\bar{Q}}_{t u}\left[G^{-1}\right]^{m n t u} \frac{\partial}{\partial x^{n}}} \\
& +\dot{\bar{Q}}_{k n} \frac{\partial}{\partial x^{j}} \bar{Q}^{m n}+\dot{\bar{Q}}_{j n} \frac{\partial}{\partial x^{k}} \bar{Q}^{m n}+\frac{\partial}{\partial \tau}\left(\bar{Q}_{k n} \frac{\partial}{\partial x^{j}} \bar{Q}^{m n}+\bar{Q}_{j n} \frac{\partial}{\partial x^{k}} \bar{Q}^{m n}\right) \\
& -\bar{Q}^{t u} \dot{\bar{Q}}_{t k}\left(\dot{\bar{Q}}_{u n} \frac{\partial}{\partial x^{j}} \bar{Q}^{m n}+\dot{\bar{Q}}_{j n} \frac{\partial}{\partial x^{u}} \bar{Q}^{m n}\right)-\left(\bar{Q}^{t u} \dot{\bar{Q}}_{t j}\left(\dot{\bar{Q}}_{u n} \frac{\partial}{\partial x^{k}} \bar{Q}^{m n}+\dot{\bar{Q}}_{k n} \frac{\partial}{\partial x^{u}} \bar{Q}^{m n}\right)\right. \\
& =\left(\frac{\dot{A}}{A}\right)\left(\delta_{j}^{m} \frac{\partial}{\partial x^{k}}+\delta_{k}^{m} \frac{\partial}{\partial x^{j}}\right)+\left(\delta_{j}^{m} \frac{\partial}{\partial x^{k}}+\delta_{k}^{m} \frac{\partial}{\partial x^{j}}\right) \frac{\partial}{\partial \tau} \\
& {\left[C_{Q}\right]_{j k}^{m n}=-\frac{1}{2} \bar{Q}_{j k} \frac{\partial}{\partial \tau} \bar{Q}^{m n}-\bar{Q}^{m r} \bar{Q}^{n s} \dot{\bar{Q}}_{r j} \dot{\bar{Q}}_{s k}+\frac{1}{4} \bar{Q}_{j k} \dot{\bar{Q}}_{r s} \dot{\bar{Q}}_{t u} \bar{Q}^{n s}\left[G^{-1}\right]^{t u r m}-\frac{1}{4} \bar{Q}_{j k} \dot{\bar{Q}}_{r s}\left[G^{-1}\right]^{m n r s} \frac{\partial}{\partial \tau}} \\
& +\bar{D}_{j} \bar{D}_{k} \bar{Q}^{m n}+\frac{1}{2}\left[G^{-1}\right]^{m n r s} \bar{D}_{r} \bar{D}_{s} \\
& =-4\left(\frac{\dot{A}}{A}\right) \delta_{j}^{m} \delta_{k}^{n}+\bar{D}_{j} \bar{D}_{k} \bar{Q}^{m n}+\frac{1}{2}\left[G^{-1}\right]^{m n r s} \bar{D}_{r} \bar{D}_{s} .
\end{aligned}
$$

For the derivation of $\left[C_{Q}\right]_{j k}^{m n}$ we used that $R^{m n}$ vanishes in the case of FRW and we also employed several times the identity $Q_{m n}\left[G^{-1}\right]^{j k m n}=-2 Q^{j k}$. Recall that the coefficient $\left[B_{Q}\right]_{j k}$ is associated with the perturbation of the last term on the right-hand side of the equation of motion for $\delta Q_{j k}$ in equation (4.25) in [1]. We mentioned already that this term is the only deviation from the standard Einstein equations that use the Hamiltonian constraint as a true Hamiltonian. However, since this term is quadratic in the shift vector $N_{j}$, it vanishes for both the background equations and the linear perturbations, when specialised to FRW. Nevertheless, we will see below that we still get small deviations from the standard treatment due to the shift vector being dynamical. Note that since $N=\sqrt{1+Q^{j k} N_{j} N_{k}}$ we get

$$
\delta N=-\frac{\bar{N}}{2} \frac{\bar{N}^{j} \bar{N}^{k}}{\bar{N}^{2}} \delta Q_{j k}+\bar{N} \frac{\bar{N}^{j}}{\bar{N}^{2}} \delta N_{j}=0 .
$$


Hence, the perturbation of the lapse function also vanishes in the context of an FRW background. Finally, we use the coefficients in equation (2.18) and obtain the following equation:

$$
\begin{aligned}
{\left[\frac{\partial^{2}}{\partial \tau^{2}}-\right.} & \left.3\left(\frac{\dot{A}}{A}\right)^{2}-\Lambda+\frac{\kappa}{4 \lambda}\left(\dot{\bar{\Xi}}^{2}-v(\bar{\Xi})\right)-\bar{D}_{m} \bar{D}_{n} \bar{Q}^{m n}+3\left(\frac{\dot{A}}{A}\right) \frac{\partial}{\partial \tau}\right] \delta Q_{j k} \\
= & {\left[-\frac{1}{A^{2}} \frac{\kappa}{2 \lambda} \dot{\Xi} \frac{\partial}{\partial \tau}+\frac{1}{A^{2}} \frac{\kappa}{4 \lambda} v^{\prime}(\bar{\Xi})\right] \delta \Xi+\left[4\left(\frac{\dot{A}}{A}\right) \delta_{(k}^{m} \frac{\partial}{\partial \tau}-2 \bar{D}_{n} \bar{D}_{(k} \bar{Q}^{m n}\right] \delta Q_{j) m} } \\
& +\left[\left(\frac{\dot{A}}{A}\right)\left(\delta_{j}^{m} \frac{\partial}{\partial x^{k}}+\delta_{k}^{m} \frac{\partial}{\partial x^{j}}\right)+\left(\delta_{j}^{m} \frac{\partial}{\partial x^{k}}+\delta_{k}^{m} \frac{\partial}{\partial x^{j}}\right) \frac{\partial}{\partial \tau}\right] \delta N_{j} \\
& +\left[-4\left(\frac{\dot{A}}{A}\right) \delta_{j}^{m} \delta_{k}^{n}+\bar{D}_{j} \bar{D}_{k} \bar{Q}^{m n}+\frac{1}{2}\left[G^{-1}\right]^{m n r s} \bar{D}_{r} \bar{D}_{s}\right] \delta Q_{m n} .
\end{aligned}
$$

Applying the derivatives to the perturbation terms, rearranging them and using equation (2.8), we end up with

$$
\begin{aligned}
\ddot{Q}_{j k}= & 2\left(\frac{\ddot{A}}{A}\right) \delta Q_{j k}+\left(\frac{\dot{A}}{A}\right) \delta \dot{Q}_{j k}-\delta_{j k} A^{2} \frac{\kappa}{2 \lambda}\left(\dot{\bar{\Xi}} \delta \dot{\Xi}-\frac{1}{2} v^{\prime}(\bar{\Xi}) \delta \Xi\right)+2\left(\frac{\dot{A}}{A}\right) \delta N_{(j, k)} \\
& +\bar{D}_{m} \bar{D}_{n} \bar{Q}^{m n} \delta Q_{j k}+\left[\bar{D}_{j} \bar{D}_{k} \bar{Q}^{m n}+\frac{1}{2}\left[G^{-1}\right]^{m n r s} \bar{D}_{r} \bar{D}_{s}\right] \delta Q_{m n}-2 \bar{D}_{n} \bar{D}_{(k} \bar{Q}^{m n} \delta Q_{j) m} .
\end{aligned}
$$

In the equation above we used that the perturbed shift vector $\delta N_{j}$ is a constant of motion which was shown in our companion paper [1] in appendix E. Therefore we have $\delta \dot{N}_{j}=0$ and thus can omit this term in equation 2.20. This equation agrees with the one for $\delta \ddot{Q}_{j k}$ in equation (B.22) if we consider that the perturbed Riemann tensor can be expressed as

$$
\delta R_{j k}=-\frac{1}{2} \bar{D}_{m} \bar{D}_{n} \bar{Q}^{m n} \delta Q_{j k}-\frac{1}{2} \bar{D}_{j} \bar{D}_{k} \bar{Q}^{m n} \delta Q_{m n}+\bar{D}_{n} \bar{D}_{(k} \bar{Q}^{m n} \delta Q_{j) m}
$$

and the perturbed Ricci scalar reduces for FRW to

$$
\delta R=\bar{R}_{m n} \delta Q^{m n}+\bar{Q}^{m n} \delta R_{m n}=\bar{Q}^{m n} \delta R_{m n}=\left[G^{1-}\right]^{m n r s} \bar{D}_{r} \bar{D}_{s} \delta Q_{m n}
$$

Likewise, in the case of the matter perturbation we obtain an additional term containing the variation of the shift vector which does not occur in the SCPT analysis. This finishes our discussion of the specialisation of the perturbed equation of motion to an FRW background. In the next section we will compare our results in detail with the one obtained in SCPT.

\section{Comparison with SCPT}

The aim of this section is to show that our invariant Hamiltonian perturbation theory reproduces the SCPT results up to small deviations. By this we mean the following:

1. SCPT Lagrangian Approach: Linearly gauge invariant, linear perturbations SCPT uses the Lagrangian formulation. It takes as matter a scalar field $\zeta$ coupled to the metric $g_{\mu \nu}$. These eleven fields are of course not invariant under (infinitesimal) spacetime diffeomorphisms of the manifold $M$ which are considered as gauge transformations. However, one constructs seven functions which are certain linear combinations built from the metric perturbations

$$
\delta g_{00}=2 a^{2} \phi, \delta g_{0 a}=a^{2}\left(S_{a}+B_{, a}\right), \delta g_{a b}=a^{2}\left[2 E_{, a b}+2 \psi \delta_{a b}+2 F_{(a, b)}+h_{a b}\right]
$$

and from the scalar field perturbation $\delta \zeta$. Here $a$ is the scale factor of the spatially flat FRW background, the vector fields $F_{a}, S_{a}$ are transversal with respect to that flat Euclidean spatial metric and the tensor $h_{a b}$ is transversal and tracefree. The seven invariants are explicitly given by

$$
\Phi:=\phi-\mathcal{H}\left(B-E^{\prime}\right)-\left(B-E^{\prime}\right)^{\prime}, \Psi:=\psi+\mathcal{H}\left(B-E^{\prime}\right), V_{a}:=S_{a}-F_{, a}, h_{a b}, Z:=\delta \zeta+\bar{\zeta}^{\prime}\left(B-E^{\prime}\right)
$$


where $\mathcal{H}=a^{\prime} / a$, a prime denotes derivation with respect to conformal time $d \eta=d t / a$ and $\bar{\zeta}$ is the background scalar field.

One then expresses the ten perturbed Einstein equations directly in terms of these seven invariants. Four of these equations, namely the temporal - temporal and the temporal - spatial equations do not contain second time derivatives of four of the seven fields, they are constraints. They imply that four of the seven invariants can be expressed in terms of the other three. The system has only three independent, linearly gauge invariant degrees of freedom. Specifically, the transversal part of the temporal - spatial part of those equations imposes $V_{a}=0$, while its longitudinal part together with the temporal - temporal equation allows us to express, for instance, $\Phi, Z$ in terms of $\Psi$. Namely, since the cosmological constant term and the energy momentum tensor have no contribution of the form $f_{, a b}$ it follows immediately 1 from the third equation in (A.54) that $\Phi=\Psi$. From the longitudinal part of the temporal - spatial equations we then immediately find that $Z+4 \lambda\left(\Psi^{\prime}+\mathcal{H} \Psi\right) / \kappa$ is a spatial constant which then must vanish due to the boundary conditions.

Thus the system of a scalar field coupled to the geometry has only the three degrees of freedom $h_{a b}, \Psi$ as independent, linearly invariant, propagating degrees of freedom. Without $Z$ we even have $\Phi=\Psi=0$.

2. Hamiltonian Approach: Manifestly invariant, linear perturbations

Our approach is completely different: first of all we work in the Hamiltonian framework. Secondly, we add additional four dust scalar fields. These are altogether fifteen configuration degrees of freedom to begin with and they are accompanied by the corresponding canonical momenta. Thus, from the very outset the number of degrees of freedom is very different compared to the SCPT framework. The components $g_{00}, g_{0 a}$ of the metric have the same information content as lapse and shift functions $n, n_{a}$ respectively. Their conjugate momenta are constrained to vanish by the primary constraints, hence $n, n_{a}$ are pure gauge and can be considered as Lagrange multipliers in the Hamiltonian formalism. This is in agreement with the Lagrangian formalism where $\delta g_{00}=2 a^{2} \phi, \delta g_{0 a}=a^{2}\left(B_{, a}+S_{a}\right)$, which are also pure gauge and are used to construct the gauge-invariant functions $\Phi, \Psi, V_{a}, h_{a b}$. Thus, the number of physical configuration degrees of freedom is reduced by four. In addition there are the secondary Hamiltonian and spatial diffeomorphism constraints. These reduce the number of physical configuration further by four. Thus, we end up with $15-8=7$ physical degrees of freedom. Without dust we also would have 3 physical degrees of freedom only, which shows that with equal matter content the Hamiltonian and Lagrangian framework are equivalent.

Indeed [19] the temporal - temporal and temporal - spatial components of the Einstein equations are proportional to the Hamiltonian and spatial diffeomorphism constraints, respectively, and the gauge transformation equations (in second order time derivative form) generated by the constraints are equivalent to the remaining spatial - spatial Einstein equations. The gauge transformations generated by the secondary constraints of the variables different from lapse and shift and their conjugate momenta (the secondary constraints, when written in terms of the momenta conjugate to the spatial metric and the scalar field, do not depend on them) are equivalent to spacetime diffeomorphisms, on shell [19]. In fact, this statement can be extended also to the lapse and shift fields [20] as we will recall in the next section.

Hence the difference between our framework and the usual one is not in the usage of the Hamiltonian versus the Lagrangian formalism but rather in the addition of the four dust fields. Now what one could do is to consider the dust - scalar - geometry system, perform linear perturbations in non gaugeinvariant quantities and then construct linearly gauge-invariant quantities from those. This we will do in the next section. However, what we will do here is to construct quantities which are fully gauge-

\footnotetext{
${ }^{1}$ We assume here that the perturbations vanish sufficiently fast at spatial infinity which is consistent with making asymptotically FRW boundary conditions. Then the Laplacian $\Delta$ has no zero modes and is therefore invertible. An equation of the form $f_{, a b}+g \delta_{a b}=0$ then implies $\Delta f+3 g=0$ and $\Delta(\Delta f+g)=0$ that is $f=g=0$.
} 
invariant to all orders and then look directly at perturbations of those manifest invariants. Hence, gauge-invariance is treated here non-perturbatively, and our perturbations themselves are manifestly gauge-invariant. This is achieved by means of deparametrisation in terms of the dust degrees of freedom, which in one stroke solves the secondary constraints and spells out the true i.e. gaugeinvariant degrees of freedom. Namely, the dust momenta can be expressed in terms of those invariants, and the dust fields themselves together with the remaining fields form non linear expressions which are fully gauge-invariant. This leaves us, before performing perturbation theory, with seven degrees of freedom, namely the invariants $\Xi, Q_{j k}$ corresponding to the scalar field $\zeta$ (or rather its pull back $\xi$ to the spatial manifold $\mathcal{X}$ ) and the spatial - spatial components of the metric together with the corresponding canonical momenta.

In terms of these seven degrees of freedom there are no longer any secondary constraints, they have been reduced. However, our equations of motion for the perturbations $\delta \Xi, \delta Q_{j k}$ of the seven perturbed manifest invariants take a form almost completely identical to those equations that we obtain in the Lagrangian formalism for the seven perturbed linear invariants $\Phi, \Psi, V_{a}, h_{a b}$, when proper identifications are made. The crucial difference arises from the fact that the latter quantities are still subject to constraints while the former are unconstrained. Yet, it turns out that while in our formalism there are no constraints any more, the would - be constraints are now constants of the motion. These constants of the motion express the influence of the dust on the system. In particular, in the limit of vanishing influence (test dust) these conservation equations turn effectively into constraint equations and then we get a precise match between the two formalisms with proper identification of variables and spatial manifolds $\mathcal{X}, \mathcal{S}$, respectively. Thus, for test dust our formalism just produces arbitrarily tiny modifications, that is, source terms, of the usual formalism although fundamentally the number of physical degrees of freedom was changed. In particular, in the late universe these modifications decay as compared to the usual terms.

It is quite remarkable that the two formalisms with fundamentally different numbers of degrees of freedom can be brought to such a close match. Looking at the details, it is hard to imagine that any material reference system other than pressure free dust can lead to such a modest modification of the usual formalism. Indeed, in the appendix of [1] we compute the modifications that arise when using instead the phantom scalar field of [5] for purposes of deparametrisation. It is shown that the modifications, in fact, grow in the late universe which is qualitatively drastically different. However, the coupling constant of the phantom and the constants of the motion can be tuned in such a way that the moment of time when the modifications become significant can be chosen to be arbitrarily late.

It is worth mentioning that since we can take the limit of 1 . vanishing dust influence (i.e. both the background energy momentum $\left(\bar{\epsilon}, \bar{\epsilon}_{j}\right)$ and its perturbation $\left(\delta \epsilon, \delta \epsilon_{j}\right)$ can be taken to zero), 2. vanishing cosmological constant, 3. vanishing physical scalar field and finally 4 . vanishing time dependence of the background FRW scale factor $a \equiv 1$, it follows immediately from our formalism that in this limit we recover also the two gravitational wave modes on Minkowski space, the other four modes are frozen out by the equations of motion.

The reader may now ask what the advantage of our more complicated formalism is compared to the usual one. There are at least three worth mentioning:

\section{A. Higher order classical general relativistic perturbation theory}

As far as standard cosmological perturbation theory is concerned, the usual formalism cannot be easily extended in a gauge invariant way to higher than linear order. The reason is that one has to construct the relevant gauge-invariant quantities at each order from scratch again. In our manifestly gauge-invariant formalism, by contrast, those quantities are simply given by the relevant higher-order perturbation of the metric and matter degrees of freedom. This is a tremendous simplification. We expect that this should also make it much easier to address general question of the stability of cosmological perturbation theory [23]. 


\section{B. Conceptual Improvement}

General relativity is a generally covariant field theory without true Hamiltonian. This leads to many conceptual problems such as the problem of time (gauge invariant functions do not evolve with respect to the Hamiltonian constraint(s)). The reason that in perturbation theory one still has non trivial evolution of the linear invariants is only due to the fact that one has chosen a background spacetime which provides a preferred notion of time and what one computes is evolution with respect to that background. However, fundamentally general relativity is a background independent field theory and since when going to higher order both classical perturbation theory (due to reasons of gauge invariance) and quantum perturbation theory (due to reasons of non renormalisability) fail, one is forced to adopt a non perturbative approach so that the problem of time reappears. The relational formalism solves the problem of time by determining a physical Hamiltonian (which is not constrained to vanish) from a chosen material reference system which evolves the manifest invariants. This brings the analysis back to the conceptually safe realm of a dynamical Hamiltonian system, albeit a technically complicated one.

\section{Quantum Theory}

At least for the pressure free dust chosen here, the Poisson algebra of the linear invariants is as simple as for their non invariant analogs. Therefore it is possible to adopt a reduced phase space quantisation approach [21] and to find Hilbert space representations of that algebra. This has the advantage that, for instance, in the framework of Loop Quantum Gravity the kinematical Hilbert space used there becomes now a physical Hilbert space. The constraints have completely disappeared from the screen. What remains is to quantise the complicated physical Hamiltonian [22] and to analyse its spectrum. This may be technically complicated but, conceptually, it is crystal clear. In particular, the fact that one has a physical Hamiltonian at one's disposal which by construction does not depend explicitly on some background time may improve the vacuum problem that one encounters in QFT on curved (time dependent) backgrounds. The problem with explicitly time dependent Hamiltonians is that the notion of vacuum (ground state) is time dependent which may lead to (infinite) particle production and, in particular, means that there is no unique ground state. The physical Hamiltonian, in principle, selects a preferred class of states as ground states, namely its zero eigenvalue states. It may be that zero or the minimum of the spectrum is not in the discrete (more precisely, pure point) spectrum or that the zero eigenvalue is vastly degenerate, however, at least conceptually, this appears to be an improvement.

In what follows we now turn to the proof of the above claims.

\subsection{Conservation Equations}

As shown in the appendix of [1, for any fully conserved quantity $F$ of a Hamiltonian sytem with Hamiltonian $H$, when we expand both the equations of motion and $F$ to order $n$ then $F$ is still a constant of motion up to terms of order $n+1$. In particular, for $n=1$ the coefficients of the correction vanish. This means that we can derive conservation equations for manifestly gauge-invariant perturbation theory by expanding the 
four times infinitely many constants of motion $C_{j}(\sigma), H(\sigma)$ where

$$
\begin{aligned}
C_{j}(\sigma) & =-\frac{2}{\kappa} D_{k} P_{j}^{k}+\frac{1}{\lambda} \Pi D_{j} \Xi \\
H(\sigma) & =\sqrt{C(\sigma, \tau)^{2}-Q^{j k}(\sigma, \tau) C_{j}(\sigma) C_{k}(\sigma)} \\
C & =C_{\text {geo }}+C_{\text {matter }} \\
C_{\text {geo }} & =\frac{1}{\kappa}\left[\frac{1}{\sqrt{\operatorname{det}(Q)}} G_{j k m n} P^{j k} P^{m n}-\sqrt{\operatorname{det}(Q)}[R[Q]-2 \Lambda]\right] \\
C_{\text {matter }} & =\frac{1}{2 \lambda}\left[\frac{\Pi^{2}}{\sqrt{\operatorname{det}(Q)}}+\sqrt{\operatorname{det}(Q)}\left(Q^{j k} \Xi_{, j} \Xi_{, k}+v(\Xi)\right)\right] .
\end{aligned}
$$

Let $\bar{\epsilon}_{j}(\sigma)=-\bar{C}_{j}(\sigma), \bar{\epsilon}(\sigma)=\bar{H}(\sigma)$ be the zeroth order values of the constants of motion which are obtained explicitly by inserting the FRW solution of appendix B.1 into the expressions (3.3). We now expand $\delta \epsilon_{j}:=\epsilon_{j}-\overline{\epsilon_{j}}, \delta \epsilon:=\epsilon-\bar{\epsilon}$ to first order, which then are still constants of the linearised equation of motion. We find after a short calculation (remember that $D_{k} P_{j}^{k}=\partial_{k} P_{j}^{k}-\Gamma_{k j}^{l} P_{l}^{k}$, since $P^{j k}$ is a tensor density) and using the perturbed gauge-invariant variables defined in appendix B.2

$$
\begin{aligned}
-\delta \epsilon_{j} & =-\frac{2}{\kappa}\left(A^{2} \delta P_{, k}^{j k}-2 \dot{A}\left(\delta Q_{j k, k}-\frac{1}{2} \delta Q_{k k, j}\right)\right)+\frac{1}{\lambda} \bar{\Pi} \delta \Xi_{, j} \\
\delta \epsilon & =\frac{1}{\bar{H}}\left[\bar{C} \delta C-\bar{Q}^{j k} \bar{C}_{k} \delta C_{j}-\bar{C}_{k} \bar{C}_{j} \delta Q^{j k}\right]=\delta C \\
\kappa \delta C_{\text {geo }} & =-\left[\frac{\dot{A}^{2}}{A} \delta^{j k} \delta Q_{j k}-2 \dot{A} A \delta_{j k} \delta P^{j k}+A \delta^{j k} \delta R_{j k}\right]+\Lambda A \delta^{j k} \delta Q_{j k} \\
\delta R_{j k} & =\frac{1}{2 A^{2}}\left[2 \delta Q_{l(j, k) l}-\Delta \delta Q_{j k}-\delta Q_{l l, j k}\right] \\
\lambda \delta C_{\text {matter }} & =\frac{1}{2}\left[2 \frac{\bar{\Pi}}{A^{3}} \delta \Pi-\frac{1}{2} A \bar{p} \delta^{j k} \delta Q_{j k}+A^{3} v^{\prime}(\bar{\Xi}) \delta \Xi\right] .
\end{aligned}
$$

Here we used that that the quantities $L, I$ introduced in appendix B.1 are explicitly given by $L=A^{2}$ and $I=-2 \dot{A}$. We will discuss the momentum and energy conservation equations separately in what follows.

\subsubsection{Momentum conservation equation}

We substitute the perturbed configuration variables for the perturbed momenta by using the equations of motion, see equation (B.21). Notice that $N_{j}=-C_{j} / H$ is also a constant of motion and that $\delta N_{j}=\delta \epsilon_{j} / \bar{\epsilon}$. Then we find from the first relation in (3.4)

$$
\begin{aligned}
& \delta \epsilon_{j}+\frac{\kappa A}{\bar{\epsilon}}\left[\Delta \delta \epsilon_{j}-\delta \epsilon_{k, k j}\right] \\
= & \frac{1}{\kappa}\left[A\left(\delta \dot{Q}_{j k, k}-\delta Q_{k k, j}\right)-2 \dot{A}\left(\delta Q_{j k, k}-\delta Q_{k k, j}\right)\right]-\frac{1}{\lambda} \bar{\Pi} \delta \Xi_{, j} .
\end{aligned}
$$

In order to connect (3.5) with the SCPT equations we parametrise our metric perturbations in the following way: First of all, SCPT employs conformal time $x^{0}=\eta$ for which $\bar{g}_{00}=-a^{2}$. On the other hand, we always have $g_{\tau \tau}:=-N^{2}+Q^{j k} N_{j} N_{k}=-1$, which means that we automatically work with cosmological time, that is, the eigentime of the dust. We use the letter $A$ instead of $a$ for the scale factor in what follows in order to emphasise that $A$ is an observable, rather than a gauge-dependent function. From the relation $d x^{0}=d \tau / A$ we find $g_{\tau \tau}=g_{00} / A^{2}, g_{\tau j}=g_{0 j} / A=N_{j}, g_{j k}=Q_{j k}$. Now in analogy to SCPT we introduce the following ten functions

$$
g_{00}=(-1+2 \phi) A^{2}, g_{0 j}=\left(B_{, j}+S_{j}\right) A^{2}, g_{j k}=A^{2}\left(2 \psi \delta_{j k}+2 E_{, j k}+2 F_{(j, k)}+h_{j k}\right),
$$


where the tensors $S_{j}, F_{j}, h_{j k}$ are transversal with respect to the Euclidean metric $\delta_{j k}$ and $h_{j k}$ is trace free. We can also express $\psi, E, F_{j}, h_{j k}$ as

$$
\begin{aligned}
\psi & =\frac{1}{4 A^{2}}\left(\delta Q_{k k}-\Delta^{-1} \delta Q_{j k, j k}\right) \\
E & =-\frac{1}{4 A^{2}} \Delta^{-1}\left(\delta Q_{k k}-3 \Delta^{-1} \delta Q_{j k, j k}\right) \\
E & =-\frac{1}{4 A^{2}} \Delta^{-1}\left(\delta Q_{k k}-3 \Delta^{-1} \delta Q_{j k, j k}\right) \\
F_{j} & =-\frac{1}{A^{2}} \Delta^{-1}\left(\delta Q_{j k, k}-\Delta^{-1} \delta Q_{k l, j k l}\right) \\
h_{j k} & =\delta Q_{j k}-2\left(\psi \delta_{j k}+E_{, j k}+F_{(j, k)}\right) .
\end{aligned}
$$

From $g_{\tau \tau}=-1$ we immediately see that automatically

$$
\phi=0
$$

that is, we are forced to work in partly synchronous "gauge". We write this expression in inverted commas because there is no gauge involved here, all the quantities are manifestly gauge invariant. We use this term only to make the analogy clear. In particular, since $N_{j}=-C_{j} / H$ can be expressed in terms of $Q_{j k}, \Xi$ and (via the equations of motion) their velocities, in our formalism the functions $B, S_{j}$ are not independent variables from the outset.

Let us decompose (3.5) into longitudinal $\delta \epsilon_{j}^{\|}=\Delta^{-1} \delta \epsilon_{k, k j}$ and transversal $\delta \epsilon_{j}^{\perp}=\epsilon_{j}-\epsilon_{j}^{\|}$parts, respectively

$$
\delta \epsilon_{j}^{\perp}+\frac{\kappa L^{1 / 2}}{\bar{\epsilon}} \Delta \delta \epsilon_{j}^{\perp}=\frac{1}{\kappa}\left[A\left(\delta \dot{Q}_{j k, k}-\Delta^{-1} \delta Q_{k l, k l j}\right)-2 \dot{A}\left(\delta Q_{j k, k}-\Delta^{-1} \delta Q_{k l, k l j}\right)\right] .
$$

Then we find

$$
\delta \epsilon_{j, j}=\frac{1}{\kappa}\left[A\left(\delta \dot{Q}_{j k, j k}-\Delta \delta Q_{k k}\right)-2 \dot{A}\left(\delta Q_{j k, j k}-\Delta \delta Q_{k k}\right)\right]-\frac{1}{\lambda} \bar{\Pi} \Delta \delta \Xi
$$

Next we invoke (3.6), (3.7) remembering that $\delta N_{j}=A\left(S_{j}+B, j\right)=-\delta \epsilon_{j} / \bar{\epsilon}$ and that $()=.d(.) / d \tau=\frac{1}{A}(.)^{\prime}$. Then the transversal part of (3.9) becomes simply

$$
\Delta V_{j}=-\kappa \frac{\delta \epsilon_{j}^{\perp}}{A^{2}}
$$

where we have introduced the variable $V_{j}=S_{j}-F_{j}^{\prime}$ which is the analog to the variable $V_{a}$ in the SCPT framework, see appendix $\underline{\mathrm{A}}$. We also used $f^{\prime}=a \dot{f}$ several times and, of course, $L=A^{2}$ and $I=-2 \dot{A}$. Equation (3.10) should be compared 3 with the vector contribution of the perturbation of the temporal -

\footnotetext{
${ }^{2}$ In the usual not manifestly gauge invariant formalism, synchronous gauge means $\phi=B=0$ while longitudinal gauge means $B=E=0$.

${ }^{3}$ In order to avoid confusion of the reader we point out that the individual contributions of geometry and matter to the Hamiltonian and spatial diffeomorphism constraints (without dust) $c$ and $c_{a}$ respectively are not exactly the temporal - temporal and temporal - spatial components of the Einstein and energy momentum tensor respectively, rather we have the identities

$$
\kappa c_{a}^{g r a v}=-\sqrt{\operatorname{det}(q)} X_{, a}^{\mu} n^{\nu} G_{\mu \nu}, \quad \kappa c^{g r a v}=-2 \sqrt{\operatorname{det}(q)} n^{\mu} n^{\nu} G_{\mu \nu}
$$

where $n^{\mu}$ is the unit future normal to a foliation $X$. Noticing that $n^{\mu}=\left(X_{, t}^{\mu}-n^{a} X_{, a}^{\mu}\right) / n$ and that $\delta n_{a}=\delta\left(q_{a b} n^{b}\right)=a\left(B, a+S_{a}\right) \neq$ 0 one finds

$$
\kappa \delta c_{a}=-a^{2}\left[\delta G_{0 a}+\left(B_{, a}+S_{a}\right)\left(2 \mathcal{H}^{\prime}+\mathcal{H}^{2}\right), \quad \kappa c=-2 a\left[\delta G_{00}+3 \mathcal{H}^{2}(3 \psi+\Delta E)\right]\right.
$$

The additional terms cancel when we replace $G_{\mu \nu}$ by $E_{\mu \nu}=G_{\mu \nu}+\Lambda g_{\mu \nu}-\frac{\kappa}{2} T_{\mu \nu}$ since $\bar{E}_{\mu \nu}=0$.
} 
spatial part of the Einstein equation, written in linearly gauge-invariant form, which we derived in the $2 \mathrm{nd}$ equation of (A.42), the second relation of (A.27) and the first relation of (A.50). That is

$$
\begin{aligned}
0 & =\delta \tilde{G}_{0 a}+\Lambda \delta \tilde{g}_{0 a}-\frac{\kappa}{2} \tilde{\delta} T_{0 a} \\
& =\left(-2\left(\Psi^{\prime}+\mathcal{H} \Phi\right)_{, a}-\frac{1}{2} \Delta V_{a}-\left(2 \mathcal{H}^{\prime}+\mathcal{H}^{2}\right) V_{a}\right)+\Lambda a^{2} V_{a}-\left(\frac{\kappa}{2 \lambda}\left(\bar{\zeta}^{\prime} Z_{, a}+a^{2} \lambda \bar{p} V_{a}\right)\right) \\
& =\left(-2\left(\Psi^{\prime}+\mathcal{H} \Phi\right)_{, a}-\frac{1}{2} \Delta V_{a}\right)-\left(\frac{\kappa}{2 \lambda} \bar{\zeta}^{\prime} Z_{, a}\right)
\end{aligned}
$$

where we used the spatial - spatial part of the background FRW equations (A.14), (A.15) in the second step, that is

$$
2 \mathcal{H}^{\prime}+\mathcal{H}^{2}=a^{2}\left(\Lambda-\frac{\kappa}{2} \bar{p}\right)
$$

and where $\bar{p}=\frac{1}{2 \lambda}\left(\dot{\bar{\Xi}}^{2}-v(\bar{\Xi})\right)$ (this holds with or without dust). The transversal piece of (3.14) is simply

$$
-\frac{1}{2} \Delta V_{a}=0
$$

If we compare this to (3.10) and if we identify the SCPT variable $V_{a}$ with $V_{j}$ then we see that, instead of a Laplace equation without source, we have a non-vanishing source. However, recall that $\delta \epsilon_{j}^{\perp}$ is a constant of motion. Hence, the source term decays as $1 / A^{2}$ and thus plays no role anymore in the late universe. This happens the sooner the smaller $\delta \epsilon_{j}^{\perp}$.

Next we turn to the longitudinal part of (3.9) which can be written as

$$
\psi_{, j}^{\prime}=-\frac{\kappa}{4}\left(\frac{1}{A^{2}} \delta \epsilon_{j}^{\|}+\frac{1}{\lambda} \bar{\Xi}^{\prime} \delta \Xi_{, j}\right)
$$

where we used $\bar{\Pi}=A^{3} \dot{\bar{\Xi}}=A^{2} \bar{\Xi}^{\prime}$. A direct comparison between (3.16) and SCPT should be in terms of variables which are direct analogs of the linearly invariant SCPT variables $\Psi, \Phi, Z$. These are given by $\Psi=\psi+\mathcal{H} f, \Phi=\phi-\mathcal{H} f-f^{\prime}, Z=\zeta+\bar{\zeta}^{\prime} f$ where we of course identify $\zeta$ with our $\Xi$ and where $f:=B-E^{\prime}$. Hence we should rewrite (3.16) in terms of these variables, recalling that in our formalism $\phi \equiv 0$ so that our $\Phi$ actually reads $\Phi=-\mathcal{H} f-f^{\prime}$. This leads to

$$
\left[\Psi^{\prime}+\mathcal{H} \Phi+\frac{\kappa}{4 \lambda} \bar{\Xi}^{\prime} \delta Z\right]_{, j}=\frac{\kappa}{4 A}\left(-\frac{1}{A} \delta \epsilon_{j}^{\|}+\bar{\epsilon}\left[B-E^{\prime}\right]_{, j}\right) .
$$

In order to arrive at this form of (3.16), we simply have rewritten $\psi, \Xi$ in terms of $\Psi, Z$ and made use of the gauge-invariant background FRW equations with dust (B.10), (B.11). They are an exact solution to our invariant Hamiltonian equations and allowed us to write $\kappa\left(\bar{\Xi}^{\prime}\right)^{2} /(4 \lambda)=-\left(\mathcal{H}^{\prime}-\mathcal{H}^{2}-\bar{\epsilon} \kappa\right) /(4 A)$ so that certain terms really combine to $\Phi$. We now compare this to the longitudinal part of the linearly invariant projection of the temporal - spatial part of the Einstein equations (3.13) in the SCPT framework which can be rewritten as

$$
\left[\Psi^{\prime}+\mathcal{H} \Phi+\frac{\kappa}{4 \lambda} \bar{\zeta}^{\prime} Z\right]_{, a}=0 .
$$

We see that (3.18) and (3.17) agree with each other under the made identification $\zeta=\Xi$ up to the corrections on the right hand side of (3.17). The first of these terms decays in time as $1 / A^{2}$ since $\delta \epsilon_{j}^{\|}$is a constant of motion. The second term decays only as $1 / A$ under the assumption that $B-E^{\prime}$ remain small during the evolution, an assumption that one always makes in perturbation theory. Notice that in the SCPT framework the quantities $B, E$ and also the combination $B-E^{\prime}$ is not linearly gauge-invariant. However, in our framework, all those quantities are gauge invariant to all orders from the outset so that (3.17) is a consistent relation among manifestly gauge-invariant variables. We just have written it in a form as close as possible to the equations in the usual formalism. 


\subsubsection{Energy conservation equation}

Finally, we consider the second conservation equation in (3.4) which after some algebra can be brought into the form

$$
\begin{aligned}
\delta \epsilon & =\delta C_{\text {geo }}+\delta C_{\text {matter }} \\
\delta C_{\text {geo }} & =-\frac{4 A}{\kappa}\left(-\Delta \Psi+3 \mathcal{H}^{\prime} \psi^{\prime}\right)+\frac{3 \psi+\Delta E}{\kappa}\left(2 \Lambda A^{3}-6 \mathcal{H}^{2} A\right) \\
\delta C_{\text {matter }} & =\frac{A^{3}}{2 \lambda}\left(2 \dot{\bar{\Xi}} \delta \dot{\Xi}+v^{\prime}(\bar{\Xi}) \delta \Xi\right)+A^{3}(3 \psi+\Delta E) \bar{\rho}
\end{aligned}
$$

where $\bar{\rho}=\frac{1}{2 \lambda}\left(\dot{\bar{\Xi}}^{2}+v(\bar{\Xi})\right)$. Using our FRW background equations (B.10) and (B.11) , we can simplify (3.19) to

$$
\delta \epsilon-\bar{\epsilon}(3 \psi+\delta E)=\frac{A^{3}}{2 \lambda}\left(2 \dot{\bar{\Xi}} \delta \dot{\Xi}+v^{\prime}(\bar{\Xi}) \delta \Xi\right)-\frac{4 A}{\kappa}\left(-\Delta \Psi+3 \mathcal{H}^{\prime} \psi^{\prime}\right)
$$

which now needs to be written in terms of the SCPT-like variables

$$
\Psi:=\psi+\mathcal{H} f, \quad \Phi=-\mathcal{H} f-f^{\prime}, \quad Z:=\Xi+\bar{\Xi}^{\prime} f \quad \text { with } \quad f=\left(B-E^{\prime}\right) .
$$

We do this by substituting $\psi, \Xi$ in (3.20) by $\Psi, Z$, respectively, thereby picking up the following correction term just involving $f$

$$
A\left[\frac{12}{\kappa} \mathcal{H}(\mathcal{H} f)^{\prime}-\frac{1}{2 \lambda}\left(2 \bar{\Xi}^{\prime}\left(\bar{\Xi}^{\prime} f\right)^{\prime}+A^{2} v^{\prime}\left(\bar{\Xi}^{\prime} \bar{\Xi}^{\prime} f\right)\right] .\right.
$$

We want to massage this into a form that involves $\Phi$. We have, using $A^{2} \bar{\rho}=\frac{1}{2 \lambda}\left(\left(\bar{\Xi}^{\prime}\right)^{2}+v(\bar{\Xi})\right)$,

$$
\begin{aligned}
-\frac{1}{2 \lambda}\left(2 \bar{\Xi}^{\prime}\left(\bar{\Xi}^{\prime} f\right)^{\prime}+A^{2} v^{\prime}(\bar{\Xi}) \bar{\Xi}^{\prime} f\right) & =\left(A^{2} \bar{\rho}\right)^{\prime} f+2\left(A^{2} \bar{\rho}\right) f^{\prime}-\frac{A}{\lambda}(A f)^{\prime} v(\bar{\Xi}) \\
& =\left(A^{2} \bar{\rho}\right)^{\prime} f+2\left(A^{2} \bar{\rho}\right) f^{\prime}+\frac{A^{2}}{\lambda} \Phi v(\bar{\Xi})
\end{aligned}
$$

where we used $\Phi=-(A f)^{\prime} / A$. Now we use our background FRW equation in the form

$$
3 \mathcal{H}^{2}=A^{2}\left[\Lambda+\frac{\kappa}{2}\left(\frac{\bar{\rho}-\bar{\epsilon}}{A^{3}}\right)\right]
$$

from which we can compute $\left(A^{2} \bar{\rho}\right)^{\prime}$ by taking its derivative. Combining then (3.23) and (3.22) we see that the $\frac{12}{\kappa} \mathcal{H}(\mathcal{H} f)^{\prime}$ term in (3.22) is cancelled and that the remaining terms are either proportional to $\Phi$ or to $\bar{\epsilon}$. The end result is

$$
\begin{aligned}
& \frac{1}{A}\left[\delta \epsilon-\bar{\epsilon}\left(3 \psi+\Delta E-2\left(B-E^{\prime}\right)^{\prime}+\mathcal{H}\left(B-E^{\prime}\right)\right)\right] \\
= & \frac{1}{2 \lambda}\left(2 \bar{\Xi}^{\prime} Z^{\prime}+A^{2} v^{\prime}(\bar{\Xi}) Z\right)-\frac{4}{\kappa}\left(-\Delta \Psi+3 \mathcal{H}^{\prime} \Psi^{\prime}\right)-A^{2}\left(\frac{v(\bar{\Xi})}{\lambda}+\frac{4 \Lambda}{\kappa}\right) \Phi .
\end{aligned}
$$

This should now be compared with the temporal - temporal Einstein equation, written in terms of the SCPT variables, derived in the first equation of (A.42), the first equation of (A.43) and (A.54), that is

$$
\begin{aligned}
0 & =\delta \tilde{G}_{00}+\Lambda \delta \tilde{g}_{00}-\frac{\kappa}{2} \delta \tilde{T}_{00} \\
& =2\left(-\Delta \Psi+3 \mathcal{H} \Psi^{\prime}\right)+2 a^{2} \Lambda \Phi-\frac{\kappa}{4 \lambda}\left(2 \bar{\zeta}^{\prime} Z^{\prime}+a^{2} v^{\prime}(\bar{\zeta}) Z-2 a^{2} v(\bar{\zeta}) \Phi\right) .
\end{aligned}
$$

Comparing (3.25) and (3.26), we see that the two equations agree, with proper identification of the variables, up to the correction term on the left hand side of (3.25) which is proportional to $\delta \epsilon, \bar{\epsilon}$ and the perturbations $B, E, \psi$, which in our formalism are gauge invariant. Assuming that these terms stay small during the cosmic evolution as one always does, the correction term decays as $1 / A$ and thus is negligible in the late universe. 


\subsection{Evolution Equations}

We derive the following perturbed evolution equation in (B.26):

$$
\begin{aligned}
\delta \ddot{Q}_{j k}= & \frac{\dot{A}}{A} \delta \dot{Q}_{j k}+2 \frac{\dot{A}}{A}\left(\delta N_{(j}\right)_{k)}+2 \frac{\ddot{A}}{A} \delta Q_{j k}-2 \delta R_{j k} \\
& +\delta_{j k}\left(\frac{1}{2} \delta R_{m m}-\frac{\kappa}{2 \lambda} A^{2}\left(\dot{\bar{\Xi}} \delta \dot{\Xi}-\frac{1}{2} v^{\prime}(\bar{\Xi}) \delta \Xi\right)\right) .
\end{aligned}
$$

First of all, we change the time variable again to $d x^{0}=d \tau / A$ and write $\delta Q_{j k}=A^{2} H_{j k}$, which gives

$$
\begin{aligned}
\delta \dot{Q}_{j k} & =A\left(2 \mathcal{H} H_{j k}+H_{j k}^{\prime}\right) \\
\delta \ddot{Q}_{j k} & =2\left(\mathcal{H}^{\prime}+\mathcal{H}^{2}\right) H_{j k}+3 \mathcal{H} H_{j k}^{\prime}+H_{j k}^{\prime \prime} \\
\frac{\dot{A}}{A} \delta \dot{Q}_{j k} & =\mathcal{H}\left(2 \mathcal{H} H_{j k}+H_{j k}^{\prime}\right) \\
\frac{\ddot{A}}{A} \delta Q_{j k} & =\mathcal{H}^{\prime} H_{j k} \\
\delta R_{j k} & =\frac{1}{2}\left(2 H_{l(j, k) l}-\Delta H_{j k}-H_{l l, j k}\right) .
\end{aligned}
$$

Inserting (3.28) into (3.27) and using $\delta N_{j}=A\left[B_{, j}+S_{j}\right]$ yields

$$
\begin{aligned}
2 \mathcal{H} H_{j k}^{\prime}+H_{j k}^{\prime \prime}= & 2 \mathcal{H}\left[B_{,(j}+S_{,(j}\right]_{, k)}-2 \delta R_{j k} \\
& +\delta_{j k}\left(\frac{1}{2} \delta R_{m m}-\frac{\kappa}{2 \lambda} A^{2}\left(\dot{\bar{\Xi}} \delta \dot{\Xi}-\frac{1}{2} v^{\prime}(\bar{\Xi}) \delta \Xi\right)\right) .
\end{aligned}
$$

Equation (3.29) should be compared to the spatial - spatial part of the Einstein equations written in linearly gauge invariant form. Using the third relation in (A.42), the third relation in (A.43), as well as the spatial - spatial components of (A.45), (A.50) and (A.54) gives

$$
\begin{aligned}
\delta \tilde{G}_{a b}+\Lambda \tilde{g}_{a b}-\frac{\kappa}{2} \delta \tilde{T}_{a b}= & \frac{1}{2}\left(h_{a b}^{\prime \prime}+2 \mathcal{H} h_{a b}-\Delta h_{a b}\right)-\left(2 \mathcal{H}^{\prime}+\mathcal{H}^{2}\right) h_{a b}-\left(V_{(a, b)}^{\prime}+2 \mathcal{H} V_{(a, b)}\right) \\
& +[\Phi-\Psi]_{a b}-\left[\Delta(\Phi-\Psi)+2\left(\Psi^{\prime \prime}+\left(2 \mathcal{H}^{\prime}+\mathcal{H}^{2}\right)(\Phi+\Psi)+\mathcal{H}(2 \Psi+\Phi)^{\prime}\right)\right] \delta_{a b} \\
& +\Lambda a^{2}\left(h_{a b}+2 \Psi \delta_{a b}\right) \\
& -\frac{\kappa}{2 \lambda}\left(\lambda \bar{p} a^{2} h_{a b}+2 \lambda \bar{p} a^{2} \Psi \delta_{a b}+\frac{1}{2}\left(2 \bar{\zeta}^{\prime}\left(\Phi+Z^{\prime}\right)-v^{\prime}(\bar{\zeta}) a^{2} Z\right) \delta_{a b}\right) \\
= & \frac{1}{2}\left(h_{a b}^{\prime \prime}+2 \mathcal{H} h_{a b}-\Delta h_{a b}\right)-\left(V_{(a, b)}^{\prime}+2 \mathcal{H} V_{(a, b)}\right) \\
& +[\Phi-\Psi]_{a b}-\left[\Delta(\Phi-\Psi)+2\left(\Psi^{\prime \prime}+\left(2 \mathcal{H}^{\prime}+\mathcal{H}^{2}\right) \Phi+\mathcal{H}(2 \Psi+\Phi)^{\prime}\right)\right] \delta_{a b} \\
& -\frac{\kappa}{4 \lambda}\left(2 \bar{\zeta}^{\prime}\left(\Phi+Z^{\prime}\right)-v^{\prime}(\bar{\zeta}) a^{2} Z\right) \delta_{a b} .
\end{aligned}
$$

Here we used (3.14) twice in the second step.

In what follows we decompose (3.29) into the various irreducible pieces. We will see that, in contrast to the conservation equations, maybe not very surprisingly, the evolution equations do not adopt any corrections.

\subsubsection{Tensor contribution}

In this case $H_{j k}=h_{j k}, \delta N_{j}=0$ and thus $\delta R_{j k}=-\Delta h_{j k} / 2$, so that the tracefree, transversal contribution to (3.29) reduces to

$$
-\Delta h_{j k}+2 \mathcal{H} h_{j k}^{\prime}+h_{j k}^{\prime \prime}=0
$$


This should be compared to the corresponding tensor contribution to (3.30) which is given by

$$
\frac{1}{2}\left(h_{a b}^{\prime \prime}+2 \mathcal{H} h_{a b}-\Delta h_{a b}\right)=0 .
$$

Thus we obtain an exact match upon identifying $h_{j k}$ on the dust space $\mathcal{S}$ with $h_{a b}$ on $\mathcal{X}$ which is diffeomorphic to $\mathcal{S}$.

\subsubsection{Vector contribution}

In this case $H_{j k}=2 A^{2} F_{(j, k)}$ and $B=0$. A short computation reveals that $\delta R_{j k}=0$. Hence, dropping the term proportional to $\delta_{j k}$ in (3.29) which is a scalar contribution, we find

$$
4 \mathcal{H} F_{(j, k)}^{\prime}+2 F_{(j, k)}^{\prime \prime}=2 \mathcal{H} S_{(j, k)}
$$

Using the conservation equation $\left(\delta N_{j}\right)^{\prime}=\left(A S_{j}\right)^{\prime}=0$, we find the identity

$$
\mathcal{H} S_{j}+S_{j}^{\prime}=0 \Rightarrow \mathcal{H} S_{(j, k)}+S_{(j, k)}^{\prime}=0 .
$$

Adding twice the second zero in (3.34) to (3.33) and dividing the resulting equation by two, we find

$$
2 \mathcal{H} V_{(j, k)}+V_{(j, k)}^{\prime}=0
$$

where we have again introduced $V_{j}=S_{j}-F_{j}^{\prime}$.

On the other hand the vector contribution to (3.30) is obviously

$$
V_{(a, b)}^{\prime}+2 \mathcal{H} V_{(a, b)}=0
$$

which is again an exact match when identifying $V_{a}$ and $V_{j}$. It is instructive how the conservation law (3.34) found its way into the equations in order to establish this match.

\subsubsection{Scalar contribution}

This time $H_{j k}=2\left(\psi \delta_{j k}+E_{, j k}\right)$ and $S_{j}=0$. A short calculation reveals that $\delta R_{j k}=-\left(\psi_{, j k}+\Delta \psi \delta_{j k}\right)$ and thus $\delta R_{k k}=-4 \Delta \psi$. Thus (3.29) becomes

$4 \mathcal{H}\left(\psi^{\prime} \delta_{j k}+E_{, j k}^{\prime}\right)+2\left(\psi^{\prime \prime} \delta_{j k}+E_{, j k}^{\prime \prime}\right)=2 \mathcal{H} B_{, j k}+2\left(\psi_{, j k}+\Delta \psi \delta_{j k}\right)+\delta_{j k}\left(-2 \Delta \psi-\frac{\kappa}{2 \lambda} A^{2}\left(\dot{\bar{\Xi}} \delta \dot{\Xi}-\frac{1}{2} v^{\prime}(\bar{\Xi}) \delta \Xi\right)\right)$.

Equation (3.37) is of the form $f_{, j k}+g \delta_{j k}=0$. Taking the trace and operating with $\partial_{j} \partial_{k}$, respectively, we learn that $3 g+\Delta f=0$ and $\Delta(g+\Delta f)=0$, respectively. Since, due to our boundary conditions, there are no zero modes of the Laplacian 4 , we conclude $f=g=0$. Therefore we can study the $(.) \delta_{j k}$ and $(),. j k$ pieces in (3.37) separately, which we note as

$$
\begin{aligned}
\left(4 \mathcal{H} \psi^{\prime}+2 \psi^{\prime \prime}-2 \Delta \psi\right) \delta_{j k} & =\left(-2 \Delta \psi-\frac{\kappa}{2 \lambda} A^{2}\left(\dot{\bar{\Xi}} \delta \dot{\Xi}-\frac{1}{2} v^{\prime}(\bar{\Xi}) \delta \Xi\right)\right) \delta_{j k} \\
{\left[4 \mathcal{H} E^{\prime}+2 E^{\prime \prime}-2 \mathcal{H} B-2 \psi\right]_{, j k} } & =0 .
\end{aligned}
$$

\footnotetext{
${ }^{4}$ The proof is standard: Assuming that $f$ is an at least twice differentiable function which decays at infinity at least as $1 / r$ where $r$ is an asymptotic radial variable. It follows that $f_{, j}$ is square integrable with respect to $L_{2}\left(\mathcal{S}, d^{3} \sigma\right)$. Now suppose that $\Delta f=0$. Then $0=<f, \Delta f>=-\sum_{j}\|f, j\|^{2}$ where the boundary term at the sphere at infinity drops out since $f f, j$ decays as $1 / r^{3}$. It follows that $f_{, j}=0$ a.e. and since $f$ is in particular continuous, it follows $f=$ const. But that constant must vanish since $f$ decays.
} 
Consider first the second equation. We note the conservation equation $\delta N_{j}^{\prime}=\left(A B_{, j}\right)^{\prime}=0$, which can be written in the form

$$
\mathcal{H} B_{, j}+B_{, j}^{\prime}=0 \Rightarrow \mathcal{H} B+B^{\prime}=0 .
$$

Here the second relation follows again from the fact that the Laplacian has no zero modes. It follows that

$$
\Phi:=-\left[\mathcal{H}\left(B-E^{\prime}\right)+\left(B-E^{\prime}\right)^{\prime}\right]=\mathcal{H} E^{\prime}+E^{\prime \prime} .
$$

Now also using the definition $\Psi=\psi+\mathcal{H}\left(B-E^{\prime}\right)$ and (3.39), a short calculation reveals that the second equation in (3.38) simply becomes

$$
2[-\Psi+\Phi]_{, j k}=0 .
$$

Next we consider the first equation in (3.38), which can be simplified to

$$
\left(4 \mathcal{H} \psi^{\prime}+2 \psi^{\prime \prime}\right) \delta_{j k}=-\frac{\kappa}{2 \lambda}\left(\bar{\Xi}^{\prime} \delta \Xi^{\prime}-\frac{A^{2}}{2} v^{\prime}(\bar{\Xi}) \delta \Xi\right) \delta_{j k}
$$

We write $\delta \Xi=Z-\bar{\Xi}^{\prime} f$, where $f=B-E^{\prime}$, so that on the right hand side of (3.42) $\delta \Xi$ is replaced by $Z$ plus the correction term

$$
\begin{aligned}
\frac{\kappa}{4 \lambda}\left(2 \bar{\Xi}^{\prime}\left(\bar{\Xi}^{\prime} f\right)^{\prime}-A^{2} v^{\prime}(\bar{\Xi}) \bar{X}^{\prime} f\right) & =\frac{\kappa}{4 \lambda}\left(\left(\left[\bar{\Xi}^{\prime}\right]^{2}\right)^{\prime} f+2\left(\bar{\Xi}^{\prime}\right)^{2} f^{\prime}-A^{2}(v(\bar{\Xi}))^{\prime} f\right) \\
& =\frac{\kappa}{4 \lambda}\left[\left(\left(\bar{\Xi}^{\prime}\right)^{2}-A^{2} v(\bar{\Xi})\right)^{\prime} f+2\left(\bar{\Xi}^{\prime}\right)^{2} f^{\prime}+2 A^{2} \mathcal{H} v(\bar{\Xi}) f\right] \\
& =\frac{\kappa}{4 \lambda}\left[2 \lambda\left(A^{2} \bar{p}\right)^{\prime} f+2\left(\bar{\Xi}^{\prime}\right)^{2}\left(f^{\prime}+\mathcal{H} f\right)-2 \mathcal{H}\left(\left(\bar{\Xi}^{\prime}\right)^{2}-A^{2} v(\bar{\Xi})\right) f\right] \\
& =\frac{\kappa}{4 \lambda}\left[2 \lambda\left(A^{2} \bar{p}\right)^{\prime} f-2\left(\bar{\Xi}^{\prime}\right)^{2} \Phi-4 \lambda \mathcal{H} A^{2} \bar{p} f\right] \\
& =-\frac{\kappa}{2 \lambda}\left(\bar{\Xi}^{\prime}\right)^{2} \Phi+\frac{\kappa}{2}\left[\left(A^{2} \bar{p}\right)^{\prime}-2 \mathcal{H} A^{2} \bar{p}\right] f \\
& =-\frac{\kappa}{2 \lambda}\left(\bar{\Xi}^{\prime}\right)^{2} \Phi+\left(A^{2} \Lambda-\left(2 \mathcal{H}^{\prime}+\mathcal{H}^{2}\right)\right)^{\prime} f-2 \frac{\kappa}{2} \mathcal{H} A^{2} \bar{p} f \\
& =-\frac{\kappa}{2 \lambda}\left(\bar{\Xi}^{\prime}\right)^{2} \Phi-2\left(\mathcal{H}^{\prime \prime}+\mathcal{H} \mathcal{H}^{\prime}\right) f+2\left(\Lambda-\frac{\kappa}{2} \bar{p}\right) \mathcal{H} A^{2} f \\
& =-\frac{\kappa}{2 \lambda}\left(\bar{\Xi}^{\prime}\right)^{2} \Phi-2\left(\mathcal{H}^{\prime \prime}+\mathcal{H} \mathcal{H}^{\prime}\right) f+2\left(2 \mathcal{H}^{\prime}+\mathcal{H}^{2}\right) \mathcal{H} f \\
& =-\frac{\kappa}{2 \lambda}\left(\bar{\Xi}^{\prime}\right)^{2} \Phi-2\left(\mathcal{H}^{\prime \prime}-\mathcal{H} \mathcal{H}^{\prime}-\mathcal{H}^{3}\right) f
\end{aligned}
$$

times $\delta_{j k}$. Here we have used (3.14) twice and the definition $\Phi:=-\left(f^{\prime}+\mathcal{H} f\right)$.

On the other hand, if we write $\psi=\Psi-\mathcal{H} f$ on the left hand side of (3.42) then we can replace $\psi$ by $\Psi$ plus the correction term

$$
\begin{aligned}
-2\left(2 \mathcal{H}(\mathcal{H} f)^{\prime}+(\mathcal{H} f)^{\prime \prime}\right) & =-2\left(2 \mathcal{H}(\mathcal{H} f)^{\prime}+\mathcal{H}^{\prime \prime} f+2 \mathcal{H}^{\prime} f^{\prime}+\mathcal{H} f^{\prime \prime}\right) \\
& =-2\left(\mathcal{H}(\mathcal{H} f)^{\prime}+\mathcal{H}^{\prime \prime} f+2 \mathcal{H}^{\prime} f^{\prime}+\mathcal{H}\left(f^{\prime}+\mathcal{H} f\right)^{\prime}\right) \\
& =2 \mathcal{H} \Phi^{\prime}-2\left(\mathcal{H} \mathcal{H}^{\prime} f+\mathcal{H}^{\prime \prime} f+\left(2 \mathcal{H}^{\prime}+\mathcal{H}^{2}\right) f^{\prime}\right) \\
& =2\left(\mathcal{H} \Phi^{\prime}+\left(2 \mathcal{H}^{\prime}+\mathcal{H}^{2}\right) \Phi\right)-2\left(\mathcal{H} \mathcal{H}^{\prime}+\mathcal{H}^{\prime \prime} f-\left(2 \mathcal{H}^{\prime}+\mathcal{H}^{2}\right) \mathcal{H}\right) f \\
& \left.=2\left(\mathcal{H} \Phi^{\prime}+\left(2 \mathcal{H}^{\prime}+\mathcal{H}^{2}\right) \Phi\right)-2\left(\mathcal{H}^{\prime \prime} f-\mathcal{H}^{\prime} \mathcal{H}-\mathcal{H}^{3}\right) \mathcal{H}\right) f
\end{aligned}
$$

times $\delta_{j k}$. We have again used the definition $\Phi:=-\left(f^{\prime}+\mathcal{H} f\right)$ frequently. Combining (3.43) and (3.44), we see that we can rewrite (3.42) in the form

$$
\begin{aligned}
& {\left[4 \mathcal{H} \Psi^{\prime}+2 \Psi^{\prime \prime}+2\left(\mathcal{H} \Phi^{\prime}+\left(2 \mathcal{H}^{\prime}+\mathcal{H}^{2}\right) \Phi\right)-2\left(\mathcal{H}^{\prime \prime} f-\mathcal{H}^{\prime} \mathcal{H}-\mathcal{H}^{3}\right) f\right] \delta_{j k} } \\
= & {\left[-\frac{\kappa}{2 \lambda}\left(\bar{\Xi}^{\prime} Z^{\prime}-\frac{A^{2}}{2} v^{\prime}(\bar{\Xi}) Z+\left(\bar{\Xi}^{\prime}\right)^{2} \Phi\right)-2\left(\mathcal{H}^{\prime \prime}-\mathcal{H} \mathcal{H}^{\prime}-\mathcal{H}^{3}\right) f\right] \delta_{j k} }
\end{aligned}
$$


or

$$
\left[2 \mathcal{H}(2 \Psi+\Phi)^{\prime}+2 \Psi^{\prime \prime}+2\left(2 \mathcal{H}^{\prime}+\mathcal{H}^{2}\right) \Phi\right] \delta_{j k}=-\frac{\kappa}{2 \lambda}\left[\bar{\Xi}^{\prime} Z^{\prime}-\frac{A^{2}}{2} v^{\prime}(\bar{\Xi}) Z+\left(\bar{\Xi}^{\prime}\right)^{2} \Phi\right] \delta_{j k} .
$$

Now the scalar contribution to (3.30) is evidently given by

$$
\begin{aligned}
0= & {[\Phi-\Psi]_{, a b}-\left[\Delta(\Phi-\Psi)+2\left(\Psi^{\prime \prime}+\left(2 \mathcal{H}^{\prime}+\mathcal{H}^{2}\right) \Phi+\mathcal{H}(2 \Psi+\Phi)^{\prime}\right] \delta_{a b}\right.} \\
& -\frac{\kappa}{4 \lambda}\left[2 \bar{\zeta}^{\prime}\left(\Phi+Z^{\prime}\right)-v^{\prime}(\bar{\zeta}) a^{2} Z\right] \delta_{a b} .
\end{aligned}
$$

This equation, of course, breaks into the two independent relations

$$
\Phi-\Psi=0
$$

and

$$
\left[2 \Psi^{\prime \prime}+2\left(2 \mathcal{H}^{\prime}+\mathcal{H}^{2}\right) \Phi+\mathcal{H}(2 \Psi+\Phi)^{\prime}\right] \delta_{a b}=-\frac{\kappa}{4 \lambda}\left[2 \bar{\zeta}^{\prime}\left[\Phi+Z^{\prime}\right]-v^{\prime}(\bar{\zeta}) a^{2} Z\right] \delta_{a b}
$$

They are obviously equivalent to (3.41) and (3.46) under the identification of the obvious variables and $\bar{\zeta}=\bar{\Xi}$. Notice again how the conservation equation (3.38) was used in order to establish this result.

\section{Comparison with SCPT Coupled to Dust}

In the previous section we showed that our manifestly gauge-invariant formalism with dust reproduces the usual formalism with linearly invariant quantities without dust, plus small corrections. In this section we construct, on the one hand, linearly gauge-invariant quantities with dust a la SCPT which are certain linear combinations of the dust, scalar and geometry perturbations. On the other hand, we expand our manifestly gauge-invariant variables, which are certain non-linear aggregates made out of the non-invariant dust, scalar and geometry perturbations, to linear order. We will show that the sets of these two quantities coincide precisely.

The original action that we started with in the companion paper [1] is the action of the physical system "gravity + scalar field + dust". Then we rewrote the Hamiltonian and diffeomorphism constraints in an equivalent form such that the Hamiltonian constraint could be deparametrised and constructed the manifestly gauge-invariant observables $Q_{i j}, P^{i j}$ and $\Xi, \Pi$, corresponding to the gauge-variant three metric $q_{a b}$, the scalar field $\xi$ and the conjugated momenta thereof. As a second step, the equations of motion for $Q_{i j}, P^{i j}$ and $\Xi, \Pi$ were perturbed around a flat FRW background, resulting in second order time derivative equations of motion for the perturbations of the metric, denoted by $\delta Q_{i j}$, and the scalar field $\delta \Xi$.

We discussed in the last section that, although starting with a system including the dust (and thus four more configuration degrees of freedom), the corresponding equation of motion for the physical degrees of freedom reproduce the results obtained by SCPT. As the latter does not employ dust, it deals only with 3 physical degrees of freedom.

In this section we want to consider the system "gravity + scalar field + dust" as our starting point again, but then follow the lines along the SCPT analysis and show that we can also use the perturbations of the dust fields, denoted by $\delta T, \delta S^{k}$, in order to construct an (up to linear order) gauge-invariant version of the perturbed four metric $\delta g_{\mu \nu}$. In this case, we have also 7 physical degrees of freedom in the system $(6$ gravitational and 1 matter). As a first step, we will use the dust fields $\delta T$ and $\delta S^{k}$ in order to derive ten gauge-invariant components of $\delta g_{\mu \nu}$ and one for the scalar field $\delta \xi$. Throughout the section we will denote an (up to linear order) gauge-invariant extension of a perturbed quantity $\delta f$ by $\delta \tilde{f}$. Secondly, we will demonstrate that 6 physical degrees of freedom contained in $\delta \widetilde{g}_{\mu \nu}$ and $\delta \widetilde{\xi}$ agree with our observables constructed in the previous sections, if these are expanded up to linear order in the non-invariant perturbations of the 
configuration variables.

As was done in the last section, we will denote an infinitesimal gauge transformation 5 by $x^{\mu} \mapsto x^{\mu}+u^{\mu}$ where $u=\left(u^{0}, u^{a}\right)$. Here $u^{a}=u_{\perp}^{a}+\delta^{a b} u_{, b}$ is a proper three vector and the scalar function $u$ is the solution of the equation $u_{, a b} \delta^{b a}=u_{, a}^{a}$. Let us recall the transformation behavior of the single components of the perturbed four metric $\delta g_{\mu \nu}$ under such an infinitesimal gauge transformation. Throughout the section we will indicate background quantities with a bar such that $\delta f:=f-\bar{f}$ for any quantity $f$. For the moment we will not decompose $\delta g_{\mu \nu}$ into scalar, vector and tensor modes, because the whole analysis done in this section can be applied to the undecomposed tensor quantities. Denoting the gauge transformed quantities by $\delta g_{\mu \nu}^{u}$, we have

$$
\begin{aligned}
& \delta g_{00}^{u}=\delta g_{00}+\left(\mathcal{L}_{u} \bar{g}\right)_{00}=\delta g_{00}-2 a\left(a u^{0}\right)^{\prime} \\
& \delta g_{0 a}^{u}=\delta g_{0 a}+\left(\mathcal{L}_{u} \bar{g}\right)_{0 a}=\delta g_{0 a}+a^{2}\left(-u_{, a}^{0}+\left(u_{\perp}^{b}+u^{, b}\right)^{\prime} \delta_{a b}\right) \\
& \delta g_{a b}^{u}=\delta g_{a b}+\left(\mathcal{L}_{u} \bar{g}\right)_{a b}=\delta g_{a b}+a^{2}\left(2 \frac{a^{\prime}}{a} u^{0} \delta_{a b}+\left(u_{\perp}^{c}+u^{, c}\right)_{, a} \delta_{c b}+\left(u_{\perp}^{c}+u^{, c}\right)_{, b} \delta_{a c}\right),
\end{aligned}
$$

where the $f^{\prime}$ denotes a derivative with respect to conformal time, denoted by $x_{0}$ and defined through $d x_{0}=a^{-1} d t$.

\subsection{Review of the SCPT Strategy for "Gravity + Scalar Field"}

Before proceeding to the case of physical system plus dust, for the benefit of the reader let us briefly recall how the (up to linear order) gauge-invariant quantities are constructed in SCPT, in the case where no dust is present. A detailed revision of this construction can be found in appendix A of this paper. As a first step, one decompose the metric into scalar components denoted by $\phi, \psi, B, E$, vector components $F_{a}, S_{a}$ and tensor components denoted by $h_{a b}$, where the latter is not of much concern, since it is already gauge-invariant. Their corresponding perturbed line element is then given by

$$
\left.\delta d s^{2}=\delta g_{\mu \nu} d x^{\mu} d x^{\nu}=a^{2}\left[2 \phi d \eta^{2}+2\left(B_{, a}+S_{a}\right) d x^{a} d \eta+\left(2 \Psi \delta_{a b}+2 E_{, a b}\right)+F_{a, b}+F_{b, a}+h_{a b}\right) d x^{a} d x^{b}\right],
$$

from which we can read off the scalar, vector and tensor components of $\delta g_{\mu \nu}$. Considering how $\delta g_{\mu \nu}$ transforms, we can derive the transformation behavior of $\phi, \psi, E, B, F_{a}, S_{a}$ and (for completeness) $h_{a b}$. It is given by

$$
\begin{aligned}
\phi^{u} & =\phi-\mathcal{H} u^{0}-\left(u^{0}\right)^{\prime}, \quad \psi^{u}=\psi+\mathcal{H} u^{0} \\
E^{u} & =E+u, \quad B^{u}=B+u^{\prime}-u^{0} \\
S_{a}^{u} & =S_{a}+\left(u_{\perp}^{b}\right)^{\prime} \delta_{a b}, \quad F_{a}^{u}=F_{a}+u_{\perp}^{b} \delta_{a b} \\
h_{a b}^{u} & =h_{a b}
\end{aligned}
$$

where we introduced $\mathcal{H}:=a^{\prime} / a$. The strategy to construct gauge-invariant quantities in SCPT is (i) take the combination $B-E^{\prime}$, which undergoes a shift in $-u^{0}$ under a gauge transformation. This combination can then be used to construct gauge-invariant quantities for $\phi$ and $\psi$. Recalling that we denote the (up to linear order) gauge-invariant version of any quantity $f$ by $\tilde{f}$, we obtain

$$
\widetilde{\phi}:=\Phi=\phi-\mathcal{H}\left(B-E^{\prime}\right)-\left(B-E^{\prime}\right)^{\prime} \quad \text { and } \quad \widetilde{\psi}:=\Psi=\psi+\mathcal{H}\left(B-E^{\prime}\right) .
$$

\footnotetext{
${ }^{5}$ Note, that in 9] the letter $\xi$ is used for denoting their infinitesimal gauge transformation. Since $\xi$ is already taken by the gauge-variant version of our scalar field, we decided to use the letter $u^{\mu}$ here, which should not be confused with the dust four-velocity given by capital $U^{\mu}$.
} 
(ii) One constructs a gauge-invariant vector by using the combination

$$
\widetilde{v}_{a}:=V_{a}=S_{a}-F_{a}^{\prime} .
$$

Consequently, we see that for the construction of gauge-invariant quantities in SCPT it is convenient to decompose $\delta g_{\mu \nu}$ into scalar, vector and tensor parts, such that four unphysical components of $\delta g_{\mu \nu}$, namely $E, B, S_{a}$ can be used to make the remaining six gauge-invariant 6 . For the benefit of the reader we also mention the capital letters $\Phi, \Psi, V_{a}$, used in the last section and in [9]. However, in this section we will use the ${ }^{\sim}$ notation for (up to linear order) gauge-invariant quantities instead, because, when considering the gauge invariant quantity of $\delta g_{\mu \nu}$, we prefer not to use $\delta G_{\mu \nu}$ to avoid possible confusion with the ordinary Einstein tensor.

\subsection{Analysis for "Gravity + Scalar field + Dust" in Analogy with SCPT}

Let us now consider the system "gravity + scalar field + dust", for which we have 4 additional degrees of freedom, compared to the system "gravity + scalar field". In this case it is very natural to first keep all components of the perturbed metric $\delta g_{\mu \nu}$ and construct their gauge-invariant extension not by means of certain scalar/vector components of the four metric, but taking the four dust fields $\left(\delta T, \delta S^{k}\right)$ to perform this task. Since we want to derive $\delta \widetilde{g}_{\mu \nu}$, there is also no need to consider the decomposed expression of that tensor. Therefore, after having recalled SCPT for the case of no dust, we will go back to equation (4.1), where the gauge-transformed components of $\delta g_{\mu \nu}$ are displayed. In the following, we will show that the behavior of $\left(\delta T, \delta S^{j}\right)$ under infinitesimal gauge transformations suggests in a natural way that these fields can be used in order to make $\delta g_{\mu \nu}$ gauge-invariant. For this purpose, we first need an Ansatz for the background dust fields $\left(\bar{T}, \bar{S}^{k}\right)$ compatible with the FRW symmetries. This can be derived from an Ansatz of the dust energy momentum tensor $\bar{T}_{\mu \nu}^{\mathrm{D}}$, specialised to the case of an FRW background. Recall that the general energy-momentum tensor was given by

$$
T_{\mu \nu}^{\mathrm{D}} \approx \rho U_{\mu} U_{\nu} \quad \text { with } \quad U_{\mu} \approx-T_{, \mu}+\left(\frac{P_{j}}{P}\right) S_{, \mu}^{j},
$$

where $\approx$ indicates weak equivalence. That means that this equations is only true up to second-class constraints. $U_{\mu}$ denotes the four-velocity of the dust, while $P_{j}, P$ are the momenta conjugated to $T, S^{j}$, respectively. Note that here $x^{\mu}=\left(t, x^{a}\right)$, since we have not yet transformed to conformal time $x^{0}$.

By considering the most general Ansatz for $\bar{T}_{\mu \nu}^{\mathrm{D}}$ that respects the FRW symmetries, we get conditions on the dust four-velocity which carry over to the dust fields $\bar{T}, \bar{S}^{k}$. The most general Ansatz is given by

$$
\bar{T}_{t a}^{\mathrm{D}}=\rho^{\mathrm{D}} \bar{U}_{0} \bar{U}_{a} \stackrel{!}{=} 0 \quad \text { and } \quad \bar{T}_{a b}^{\mathrm{D}}=\rho^{\mathrm{D}} \bar{U}_{a} \bar{U}_{b} \propto \delta_{a b} .
$$

In order to satisfy these requirements, we make an Ansatz for the dust fields $\left(\bar{T}, \bar{S}^{k}\right)$. For $\bar{T}:=\bar{T}(t)$ we take a, so far, arbitrary function of $t$ so that $\bar{T}_{, a}=0$. For $\bar{S}^{k}=\bar{S}^{k}(x, y, z)$ we assume that it does not depend on time, so that $\bar{S}_{, t}^{k}=0$. Furthermore, we require that $\bar{S}_{, a}^{k}$ respects the homogeneity and isotropy requirements, that means $\bar{S}_{, a b}^{k}=0$. Therefore $\bar{S}^{k}=M_{a}^{k} x^{a}$ can only be linear in the spatial coordinates $x^{a}$, where $M_{a}^{k}$ is a matrix with constant entries. From the dust equation of motion shown in [1] in section 2, we know that $\mathcal{L}_{\bar{U}} \bar{S}_{, \mu}^{k}=0$. Inserting the Ansatz for $\bar{T}, \bar{S}^{k}$ yields $\bar{U}^{a}=1 / a^{2} \bar{U}_{a}=0$. On the other hand, we have $\bar{U}_{a}:=-\bar{T}_{, a}-\frac{\bar{P}_{k}}{\bar{P}} \bar{S}_{, a}^{k}=-\frac{\bar{P}_{k}}{\bar{P}} \bar{S}_{, a}^{k}$. One of our basic assumptions is that the matrix $\bar{S}_{, a}^{k}$ with $k, a=1,2,3$ is invertible and we denoted its inverse by $\bar{S}_{k}^{a}$. Requiring now that $\bar{U}_{a}=-\frac{\bar{P}_{k}}{\bar{P}} \bar{S}_{, a}^{k} \stackrel{!}{=} 0$ and multiplying by the inverse $\bar{S}_{j}^{a}$ on both sides yields the condition $-\frac{\bar{P}_{j}}{\bar{P}}=0$ for all $j=1,2,3$. Hence, the conjugated momentum $\bar{P}_{k}$ of $\bar{S}^{k}$ vanishes in the case of FRW if $\bar{T}$ carries all the time dependence and $\bar{S}^{k}$ all the spatial dependence

\footnotetext{
${ }^{6}$ Recall that the vectors $S_{a}, F_{a}$ are assumed to be divergenceless and therefore carry only two degrees of freedom.
} 
of $\bar{U}_{\mu}$. Furthermore, from the fact that $\bar{T}$ defines proper time along the dust lines, i.e. $\quad \mathcal{L}_{\bar{U}} \bar{T}=1$ as a consequence of the dust equation of motion, we get $\left(\bar{T}_{, t}\right)^{2}=1$, that is $\bar{T}_{, t}=1$. Hence, we obtain $\bar{T}=t$. Thus, the only non-vanishing component of $T_{\mu \nu}^{\mathrm{D}}$ is the $t t$-component

$$
\bar{T}_{t t}^{\mathrm{D}}=\rho^{\mathrm{D}}, \quad \bar{T}_{t a}^{\mathrm{D}}=\bar{T}_{a b}^{\mathrm{D}}=0 .
$$

Thus, the symmetry requirements for $\bar{T}_{\mu \nu}^{\mathrm{D}}$ lead to the usual four-velocity of pressure-free dust given by $\bar{U}_{\mu}=(-1,0,0,0)$.

Now, we want to analyse how the perturbed dust fields $\left(\delta T, \delta S^{k}\right)$, defined through $\delta T:=T-\bar{T}$ and $\delta S^{k}:=S^{k}-\bar{S}^{k}$, behave under a gauge transformation $x^{\mu} \rightarrow x^{\mu}+u^{\mu}$. The quantities $\left(\delta T, \delta S^{k}\right)$ will, for instance, be included in the perturbed dust four-velocity $\delta U_{\mu}$ and thus in the perturbed energy momentum tensoi $T_{\mu \nu}^{\mathrm{D}}$. For this purpose we will work, similarly to SCPT, in conformal time $d x^{0}=a^{-1} d t$. Then we have $\bar{T}_{, 0}=a \bar{T}_{, t}=a$. Hence, the perturbations are given by $\delta T=T\left(t\left(x^{0}\right)\right)-\bar{T}\left(t\left(x^{0}\right)\right)$ and $\delta S^{k}=S^{k}-\bar{S}^{k}=S^{k}-M_{a}^{k} x^{a}$. A gauge transformation $u$ acts on $\delta T$ as

$$
\delta T^{u}=\delta T+\mathcal{L}_{u} \bar{T}=\delta T+u^{0} \bar{T}_{, 0}=\delta T+u^{0} \bar{T}^{\prime}=\delta T+a u^{0}
$$

where' denotes derivative with respect to conformal time $x^{0}$ as introduced already above. We can see that $a^{-1} \delta T$ is simply shifted by $u^{0}$. In the last step we used that $\bar{T}^{\prime}=\bar{T}_{, 0}=a$. in [9] the authors use the combination $B-E^{\prime}$ which undergoes a shift of $-u^{0}$ under gauge transformations. Hence, in order to use the perturbations of the dust field, we can simply take $a^{-1} \delta T$ which is able to compensate any shift of $u^{0}$ in $\delta g_{\mu \nu}^{u}$ in equation (4.1). The other shift which occurs for the components $\delta g_{o a}^{u}$ and $\delta g_{a b}^{u}$ are certain derivatives of the three vector $u^{a}$. This shift can be compensated by the transformation behaviour of $\delta S^{k}$ which is shown below

$$
\delta\left(S_{, a}^{k}\right)^{u}=\delta S_{, a}^{k}+\left(\mathcal{L}_{u} \bar{S}^{k}\right)_{, a}=\delta S_{, a}^{k}+u^{b} \bar{S}_{, a b}^{k}+u_{, a}^{b} \bar{S}_{, b}^{k}=\left[\delta S^{k}+u^{b} \bar{S}_{, b}^{k}\right]_{, a} .
$$

Here we used that $\bar{S}^{k}$ has no conformal time dependence. Consequently, the $\delta S^{k}$ themselves transform as

$$
\delta\left(S^{k}\right)^{u}=\delta S^{k}+u^{b} \bar{S}_{, b}^{k}=\delta S^{k}+\left(u_{\perp}^{b}+\delta^{b c} u_{, c}\right) \bar{S}_{, b}^{k},
$$

which is the usual transformation rule for scalars under such a gauge transformation. Furthermore, we used the decomposition of $u^{b}$ into a longitudinal and transversal part mentioned before. From the equation above we can read off that the combination $\delta S^{k} \bar{S}_{k}^{b} \delta_{a b}$ will be shifted by the vector $u_{a}$ after having applied a gauge transformation. Therefore, this combination can be used to compensate for all shifts in $u_{a}$ and derivatives thereof which occur in the gauge-transformed components of $\delta g_{0 a}$ and $\delta g_{a b}$. Hence, the perturbed dust fields $\left(\delta T, \delta S^{k}\right)$ suggest very naturally a way to construct the (up to linear order) gauge-invariant metric perturbations $\delta \widetilde{g}_{\mu \nu}$, since $\left(a^{-1} \delta T, \bar{S}_{k}^{b} \delta S^{k} \delta_{a b}\right)$ exactly provide the shifts in $u^{0}$ and $u^{a}$, respectively, which are needed. The components of $\delta \widetilde{g}_{\mu \nu}$ are, in particular, given by

$$
\begin{aligned}
\delta \widetilde{g}_{00} & =\delta g_{00}+2 a(\delta T)_{, 0}=\delta g_{00}+2 a(\delta T)^{\prime} \\
\delta \widetilde{g}_{o a} & =\delta g_{0 a}+a^{2}\left[a^{-1}(\delta T)_{, a}-\left(\bar{S}_{k}^{b} \delta S^{k}\right)_{, 0} \delta_{a b}\right]=\delta g_{0 a}+a^{2}\left[a^{-1}(\delta T)_{, a}-\left(\bar{S}_{k}^{b} \delta S^{k}\right)^{\prime} \delta_{a b}\right] \\
\delta \widetilde{g}_{a b} & =\delta g_{a b}-a^{2}\left[2 \frac{a^{\prime}}{a^{2}} \delta T \delta_{a b}+\left(\bar{S}_{k}^{c} \delta S^{k}\right)_{, a} \delta_{b c}+\left(\bar{S}_{k}^{c} \delta S^{k}\right)_{, b} \delta_{a c}\right] .
\end{aligned}
$$

Note that all ten components of $\delta \widetilde{g}_{\mu \nu}$ are gauge-invariant now. These gauge-invariant combinations are different from the usual SCPT analysis where, as mentioned above, 4 out of the ten metric components are used to make the remaining six components gauge-invariant. Upon including the dust, the analysis

\footnotetext{
${ }^{7}$ Note that, due to the vanishing of the background term $\bar{P}_{k} / \bar{P}$, the expression $\delta S_{, \mu}^{k}$ occurs in $\delta U_{\mu}$ only formally, since it is multiplied by the vanishing background quantity $\bar{P}_{k} / \bar{P}$.
} 
above shows that the dust is a natural candidate for the unphysical degrees of freedom, because their changes under gauge transformation can in a simple way compensate for the changes of the components of $\delta g_{\mu \nu}$. A consequence is that when decomposing $\delta \widetilde{g}_{\mu \nu}$ into scalar, vector and tensor modes, we also obtain gauge-invariant extensions of the quantities $E, B, S_{a}, F_{a}$. For completeness and to facilitate comparison with the standard SCPT framework, we also list the gauge-invariant extensions of these decomposed quantities $\widetilde{\phi}, \widetilde{\psi}, \widetilde{E}, \widetilde{B}, \widetilde{S}_{a}, \widetilde{F}_{a}$ for the case where the dust fields are used to construct those gauge-invariant quantities. For this purpose, we need to decompose the vector $\delta w^{c}:=\bar{S}_{k}^{c} \delta S^{k}$ into its longitudinal and transversal part, thus $\delta w^{c}=\delta w_{\|}^{c}+\delta w_{\perp}^{c}=\delta^{c d} \delta w_{\|, c}+\delta w_{\perp}^{c}$, where $\delta w_{\|}$is the solution of the equation $\Delta \delta w_{\|}=\delta w_{, c}^{c}$. Explicitly, we have

$$
\begin{array}{rlrl}
\widetilde{\phi} & =\Phi=\phi+\mathcal{H} a^{-1} \delta T+\left(a^{-1} \delta T\right)^{\prime} & \widetilde{\psi}=\Psi=\psi-\mathcal{H} a^{-1} \delta T \\
\widetilde{E}=E-\delta w_{\|} & \widetilde{B}=B+a^{-1} \delta T-\left(\delta w_{\|}\right)^{\prime} \\
\widetilde{S}_{a}=S_{a}-\left(\delta w_{\perp}^{b}\right)^{\prime} \delta_{a b} & \widetilde{F}_{a}=F_{a}-\delta w_{\perp}^{b} \delta_{a b} .
\end{array}
$$

Finally, in order to derive the gauge-invariant extension of the scalar field perturbation $\delta \widetilde{\xi}$, we consider first the energy momentum tensor of the unperturbed scalar field $\xi$, given by

$$
\lambda T_{\mu \nu}^{\text {matter }}=\left[\nabla_{\mu} \xi\right]\left[\nabla_{\nu} \xi\right]-\frac{1}{2} g_{\mu \nu}\left[g^{\rho \sigma}\left[\nabla_{\rho} \xi\right]\left[\nabla_{\sigma} \xi\right]+v(\xi)\right] .
$$

Here $v(\xi)$ denotes the potential of the scalar field. Specialising to an FRW background as discussed in appendix A, we obtain for the components of $\bar{T}_{\mu \nu}$

$$
\begin{aligned}
\bar{T}_{00}^{\text {matter }} & =\frac{1}{2}\left[\left(\bar{\xi}^{\prime}\right)^{2}+a^{2} v(\bar{\xi})\right]=a^{2} \bar{\rho}^{\text {matter }} \\
\bar{T}_{0 a}^{\text {matter }} & =0 \\
\bar{T}_{a b}^{\text {matter }} & =\frac{1}{2} \delta_{a b}\left[\left(\bar{\xi}^{\prime}\right)^{2}-a^{2} v(\bar{\xi})\right]=a^{2} \bar{p}^{\text {matter }},
\end{aligned}
$$

where we used $\bar{\rho}^{\text {matter }}=\frac{1}{2}\left(\dot{\xi}^{2}+v(\xi)\right)$ and $\bar{p}^{\text {matter }}=\frac{1}{2}\left(\dot{\xi}^{2}-v(\xi)\right)$. The linear perturbation $\delta T_{\mu \nu}^{\text {matter }}$ of the $\bar{T}_{\mu \nu}^{\text {matter }}$ components are

$$
\begin{aligned}
\delta T_{00}^{\text {matter }} & =\frac{1}{2 \lambda}\left[2 \bar{\xi}^{\prime} \delta \xi^{\prime}+a^{2} v^{\prime}(\bar{\xi}) \delta \xi-v(\bar{\xi}) \delta g_{00}\right] \\
\delta T_{0 a}^{\text {matter }} & =\frac{1}{\lambda}\left[\bar{\xi}^{\prime} \delta \xi_{, a}+\lambda \bar{p} \delta g_{0 a}\right] \\
\delta T_{a b}^{\text {matter }} & =\frac{1}{\lambda}\left[\lambda \bar{p} \delta g_{a b}+\frac{1}{2} \delta_{a b}\left[\frac{1}{a^{2}}\left(\bar{\xi}^{\prime}\right)^{2} \delta g_{00}+2 \bar{\xi}^{\prime} \delta \xi^{\prime}-a^{2} v_{, \bar{\xi}}(\bar{\xi}) \delta \xi\right]\right] .
\end{aligned}
$$

Here we introduced $v_{\bar{\xi}}:=d v / d \bar{\xi}$. The gauge-invariant extension of $\delta T_{\mu \nu}^{\text {matter }}$, denoted by $\delta \widetilde{T}_{\mu \nu}^{\text {matter }}$, can then be constructed by replacing the quantities $\delta g_{00}, \delta g_{0 a}$ in equation (4.12) and $\delta \xi$ by their gauge-invariant extension. We have not worked out the latter yet, so we need to consider how $\delta \xi$ transforms under a gauge transformation. This is simply given by

$$
\delta \xi^{u}=\delta \xi+\left(\mathcal{L}_{u} \bar{\xi}\right)=\delta \xi+u^{0} \bar{\xi}_{, 0}=\delta \xi+u^{0} \bar{\xi}^{\prime} .
$$

Consequently, the (up to linear order) gauge-invariant extension of $\delta \xi$ is simply

$$
\delta \widetilde{\xi}=\delta \xi-a^{-1} \bar{\xi}^{\prime} \delta T .
$$

Thus, the components of $\delta \widetilde{T}_{\mu \nu}^{\text {matter }}$ are

$$
\begin{aligned}
\delta \widetilde{T}_{00}^{\text {matter }} & =\frac{1}{2 \lambda}\left[2 \bar{\xi}^{\prime} \delta \widetilde{\xi}^{\prime}+a^{2} v_{\bar{\xi}}(\bar{\xi}) \delta \widetilde{\xi}-v(\bar{\xi}) \delta \widetilde{g}_{00}\right] \\
\delta \widetilde{T}_{0 a}^{\text {matter }} & =\frac{1}{\lambda}\left[\bar{\xi}^{\prime} \delta \widetilde{\xi}_{, a}+\lambda \bar{p} \delta \widetilde{g}_{0 a}\right] \\
\delta \widetilde{T}_{a b}^{\text {matter }} & =\frac{1}{\lambda}\left[\lambda \bar{p} \delta \widetilde{g}_{a b}+\frac{1}{2} \delta_{a b}\left[\frac{1}{a^{2}}\left(\bar{\xi}^{\prime}\right)^{2} \delta \widetilde{g}_{00}+2 \bar{\xi}^{\prime} \delta \widetilde{\xi}^{\prime}-a^{2} v_{, \bar{\xi}}(\bar{\xi}) \delta \widetilde{\xi}\right]\right] .
\end{aligned}
$$


This finishes our task to show that, by means of the four (perturbed) dust fields $\delta T, \delta S^{k}$, (up to linear) gauge-invariant extensions of the four metric $\delta \widetilde{g}_{\mu \nu}$, as well as of the scalar field energy momentum tensor $\delta \widetilde{T}_{\mu \nu}^{\text {matter }}$ can be constructed.

\subsection{Comparison between SCPT + Dust and our Relational Framework}

As a last step, we will demonstrate that the (up to linear order) gauge-invariant quantities $\delta \widetilde{g}_{\mu \nu}$ and $\delta \widetilde{T}_{\mu \nu}^{\text {matter }}$ derived in the last section agree with our (manifestly) gauge-invariant quantities $\delta Q_{i j}$ (+ corresponding lapse $\delta N$ and shift $\left.\delta N_{j}\right)$ and $T_{i j}^{\text {matter }}(\delta Q, \delta \Xi)$, when these are expanded up to linear order. By this we mean that we will expand the formula in [1] for the gauge-invariant quantities $F:=O_{f}$, associated with any quantity $f$, around a given phase point (background), but now in terms of the non-invariant variables. The perturbations are then defined through $\delta O_{f}:=O_{f}-O_{\bar{f}}=O_{f}-\bar{f}$, where we assume that the perturbation is around the configuration $T=\bar{T}, S^{k}=\bar{S}^{k}$. We consider only terms in which the perturbations occur at most linearly. Recall that in order to construct the (manifestly) gauge-invariant extensions of the perturbed three metric $\delta q_{a b}$, perturbed lapse function $\delta n$ and perturbed shift vector $\delta n_{a}$, we first rewrote the Hamiltonian and diffeomorphism constraint $c^{\text {tot }}=c^{\text {geo }}+c^{\text {matter }}+c^{\mathrm{D}}=c+c^{\mathrm{D}}$ and $c_{a}^{\text {tot }}=c_{a}^{\text {geo }}+c_{a}^{\text {matter }}+c_{a}^{\mathrm{D}}=c_{a}+c^{\mathrm{D}}$, respectively, in the following equivalent form:

$$
\begin{aligned}
& \tilde{c}^{\text {tot }}=P+h \quad \text { with } \quad h:=\sqrt{c^{2}-q^{a b} c_{a} c_{b}} \\
& \tilde{c}_{j}^{\text {tot }}=P_{j}+h_{j} \quad \text { with } \quad h_{j}:=S_{j}^{a}\left[-h T_{, a}+c_{a}\right] .
\end{aligned}
$$

Note that the ${ }^{\sim}$ here has nothing to do with gauge invariance. We just took the notation used before in [1. Since these constraints are mutually Poisson commuting, we could then perform our construction of the gauge-invariant extensions in two steps. First we construct diffeomorphism -invariant quantities $O_{f}^{(1)}$ and afterwards use those in order to construct quantities that are also gauge-invariant with respect to the Hamiltonian constraint denoted by $F:=O_{O_{f}^{(1)}}^{(2)}$. The corresponding perturbed quantity is then given by $\delta F:=$ $\delta\left(O_{O_{f}^{(1)}}^{(2)}\right)$. Here, we are only interested in the terms of $\delta F$ which are linear in the perturbations, because we want to compare them with (up to linear order) gauge-invariant components of $\delta \widetilde{g}_{\mu \nu}$ in equation (4.12). For this reason, the general expression for $\delta F$ simplifies a lot and can be easily calculated. For the perturbations of the diffeomorphism-invariant observables $\delta O_{\delta f}^{(1)}$ we find up to linear order in the perturbations

$$
\begin{aligned}
\delta O_{f}^{(1)} & =\delta f+\int d^{3} y\left(\bar{S}^{k}-S^{k}\right)(y) \overline{\left\{\tilde{c}_{k}^{\text {tot }}(y), f(x)\right\}} \\
& =\delta f-\int d^{3} y \delta S^{k}(y) \overline{\left\{\tilde{c}_{k}^{\text {tot }}(y), f(x)\right\}} .
\end{aligned}
$$

Here we used $\delta f=f-\bar{f}$ and, in notation introduced in [1], $\bar{S}^{k}=\sigma^{k}$, as well as the definition of $\delta S^{k}=S^{k}-\bar{S}^{k}$ in the last line. Note that up to linear order only Poisson brackets evaluated on background quantities occur, which we indicated by the bar above the Poisson bracket. This is due to the reason that $\delta S^{k}$ is already linear in the perturbations. Using $\delta O_{f}^{(1)}$, we are now able to construct $\delta F$, which up to linear terms in the perturbation given by

$$
\begin{aligned}
{[\delta F]_{\text {lin }} } & =: \delta \tilde{f} \\
& =\delta O_{f}^{(1)}+\int d^{3} y(\bar{T}-T)(y)\left\{\tilde{c}^{\mathrm{tot}}(y), \delta O_{f}^{(1)}(x)\right\} \\
& =\delta f-\int d^{3} y \delta S^{k}(y) \overline{\left\{\tilde{c}_{k}^{\mathrm{tot}}(y), f(x)\right\}}-\int d^{3} y(T-\bar{T})(y) \overline{\left\{\tilde{c}^{\mathrm{tot}}(y), f(x)\right\}} \\
& =\delta f-\int d^{3} y \delta T(y) \overline{\left\{\tilde{c}^{\mathrm{tot}}(y), f(x)\right\}}-\int d^{3} y \delta S^{k}(y) \overline{\left\{\widetilde{c}_{k}^{\mathrm{tot}}(y), f(x)\right\}}
\end{aligned}
$$


Here we denoted $[\delta F]_{\text {lin }}$, expanded up to linear order, by $\delta \tilde{f}$ as we did for $\delta g_{\mu \nu}$, in order to emphasize that, in contrast to $\delta F$, the former are not gauge-invariant up to all orders. Furthermore we used that $\bar{T}=\tau$ in our case and $\delta T=T-\bar{T}$. We want to apply the above formula to $f=q_{a b}, \xi$, respectively, both of which are Poisson commuting with $P, P_{k}$, the momenta conjugate to $T$ and $S^{k}$, respectively. Recalling the form of the constraints in equation (4.20), we see that equation (4.22) simplifies even more to

$$
\delta \tilde{f}=\delta f-\int d^{3} y \delta T(y) \overline{\{h(y), f(x)\}}-\int d^{3} y \delta S^{k}(y) \bar{S}_{k}^{a} \overline{\left\{c_{a}(y), f(x)\right\}}
$$

for those $f$ 's which do not depend on the dust variables $\left(T, S^{k}\right)$. Furthermore, we used that

$$
\overline{\left\{\left(h T_{, a}\right)(y), f(x)\right\}}=\overline{T_{, a}(y)\{h(y), f(x)\}}=\bar{T}_{, a}(y) \overline{\{h(y), f(x)\}}=0,
$$

because we have $\bar{T}_{, a}=0$. The computation of the remaining Poisson bracket above yields

$$
\begin{aligned}
\int d^{3} y \delta T(y) \overline{\{h(y), f(x)\}} & =\int d^{3} y \delta T(y)\left(\bar{n}(y) \overline{\{c(y), f(x)\}}+\bar{n}_{a}(y) \overline{\left\{c_{b}(y), f(x)\right\}}\right) \\
& =\int d^{3} y \delta T(y)(\bar{n}(y) \overline{\{c(y), f(x)\}})+\overline{\left(\mathcal{L}_{\vec{n}} f\right)}(x) \\
\int d^{3} y \delta S^{k}(y) \bar{S}_{k}^{a} \overline{\left\{c_{a}(y), f(x)\right\}} & =\overline{\left(\mathcal{L}_{\bar{S}_{k}^{a} \delta S^{k}} f\right)}(x),
\end{aligned}
$$

where we used the definition of the lapse function $n:=c / h$ and the shift vector $n_{a}:=-c_{a} / h$. Hence, the final version of the formula for $\delta \tilde{f}$ has the form

$$
\delta \tilde{f}=\delta f-\int d^{3} y \delta T(y)(\bar{n}(y) \overline{\{c(y), f(x)\}})-\overline{\left(\mathcal{L}_{\vec{n}} f\right)}-\overline{\left(\mathcal{L}_{\bar{S}_{k}^{a} \delta S^{k}} f\right)} .
$$

In order to compare the results obtained from equation (4.26) with the one for $\delta \widetilde{g}_{\mu \nu}$ derived in the last section shown in equation (4.12), we need to express the perturbations of the four metric in terms of the corresponding perturbations of the three metric $q_{a b}$, lapse function $n$ and shift vector $n_{a}$. Recall that $g_{00}=a^{2}\left(-n^{2}+q^{a b} n_{a} n_{b}\right), g_{0 a}=a n_{a}$ and $g_{a b}=q_{a b}$. Consequently, the relation for the (up to linear order) gauge-invariant perturbations is given by

$$
\begin{aligned}
\delta \widetilde{g}_{00} & =a^{2}\left(-2 \bar{n} \delta \widetilde{n}-\delta q_{a b} \bar{n}^{a} \bar{n}^{c}+2 \bar{q}^{a b} \bar{n}_{a} \delta \widetilde{n}_{b}\right)=-2 a^{2} \delta \widetilde{n} \\
\delta \widetilde{g}_{0 a} & =a \delta \widetilde{n}_{a} \\
\delta \widetilde{g}_{a b} & =\delta \widetilde{q}_{a b},
\end{aligned}
$$

where we used that $\bar{n}=1$ and $\bar{n}^{a}=0$. Starting with the spatial - spatial component of the metric $\delta q_{a b}$ and applying the formula in equation (4.26) to it, we obtain

$$
\begin{aligned}
\delta \widetilde{q}_{a b} & =\delta q_{a b}-\int d^{3} y \delta T(y)\left(\bar{n}(y) \overline{\left\{c(y), q_{a b}(x)\right\}}\right)-{\overline{\left(\mathcal{L}_{\vec{n}} q\right)}}_{a b}-{\overline{\left(\mathcal{L}_{\bar{S}_{k}^{a} \delta S^{k}} q\right)}}_{a b} \\
& =\delta q_{a b}-\int d^{3} y \delta T(y)\left(\overline{\left\{c(y), q_{a b}(x)\right\}}\right)-{\overline{\left(\mathcal{L}_{\bar{S}_{k}^{a} \delta S^{k}} q\right)}}_{a b} .
\end{aligned}
$$

Here we used in the last line that for FRW the lapse function and the shift vector are given by

$$
\bar{n}=c / h=c / \sqrt{c^{2}-q^{a} b c_{a} c_{b}}=c / c=1 \quad \text { and } \quad \bar{n}_{a}=-c_{a} / h=-c_{a} / \sqrt{c^{2}-q^{a b} c_{a} c_{b}}=0 .
$$

The remaining integral in equation (4.28) yields

$$
\begin{aligned}
\int d^{3} y \delta T(y)\left(\overline{\left\{c(y), q_{a b}(x)\right\}}\right) & =\int d^{3} y \delta T(y)\left(\overline{\frac{2 n}{\sqrt{\operatorname{det} q}}\left(q_{a c} q_{b d}-\frac{1}{2} q_{a b} q_{c d}\right) p^{c d}}\right)(x) \delta(x, y) \\
& =\delta T(x) \frac{2}{a^{3}}\left(-\frac{1}{2} a^{4}\right)(-2 \dot{a}) \delta_{a b} \\
& =\delta T(x) 2 a \dot{a} \\
& =2 a^{\prime} \delta T(x) .
\end{aligned}
$$


In the second line we inserted the explicit form of $\bar{q}_{a b}$ for FRW and the corresponding momentum $p^{a b}$ conjugate to it, given by $\bar{q}_{a b}=a^{2} \delta_{a b}$ and $p^{a b}=-2 \dot{a} \delta^{a b}$, respectively. In the last step we replaced the derivative with respect to $\bar{T}$ by one with respect to conformal time. Considering the explicit form of $\bar{q}_{a b}$ also for the Lie derivative occurring in equation (4.28), we end up with

$$
\begin{aligned}
\delta \widetilde{q}_{a b} & =\delta q_{a b}-2 a^{\prime} \delta T \delta_{a b}-a^{2}\left(\bar{S}_{k}^{c} \delta S^{k}\right)_{, a} \delta_{c b}-a^{2}\left(\bar{S}_{k}^{c} \delta S^{k}\right)_{, b} \delta_{a c} \\
& =\delta q_{a b}-a^{2}\left[2 \frac{a^{\prime}}{a^{2}} \delta T \delta_{a b}+\left(\bar{S}_{k}^{c} \delta S^{k}\right)_{, a} \delta_{c b}+\left(\bar{S}_{k}^{c} \delta S^{k}\right)_{, b} \delta_{a c}\right] .
\end{aligned}
$$

The last line agrees exactly with the expression in equation (4.12) if we identify $\delta \widetilde{g}_{a b}$ with $\delta \widetilde{q}_{a b}$. Next, we want to discuss the case for the scalar field perturbation $\delta \xi$. Since any spatial derivative of the background scalar field $\bar{\xi}$ vanishes, the Lie derivative with respect to $\bar{S}_{k}^{c} \delta S^{k}$ in equation (4.26), evaluated on the FRW background, vanishes as well. Therefore the (up to linear order) gauge-invariant extension is simply

$$
\begin{aligned}
\delta \widetilde{\xi} & =\delta \xi-\int d^{3} y \delta T(y)(\overline{\{c(y), f(x)\}}) \\
& =\delta \xi-\int d^{3} y \delta T(y)\left(\overline{\frac{n}{\sqrt{\operatorname{det} q}} \pi}\right)(x) \delta(x, y) \\
& =\delta \xi-\delta T(x)\left(\frac{1}{\sqrt{\operatorname{det}(\bar{q})}} \dot{\bar{\xi}} \sqrt{\operatorname{det} \bar{q}}\right) \\
& =\delta \xi-a^{-1} \bar{\xi}^{\prime} \delta T(x),
\end{aligned}
$$

which exactly coincides with the result in equation (4.18). Note that we reexpressed the momentum $\bar{\pi}$ conjugated to $\bar{\xi}$ in terms of $\dot{\bar{\xi}}$ in the third line, which in the case of FRW has the simply form $\bar{\pi}=\dot{\bar{\xi}} \sqrt{\operatorname{det} \bar{q}}$.

Finally we have to discuss the lapse function $\delta n$ and the shift vector $\delta n^{a}$. We want to stress that in the rest of these two papers we always have treated them as pure gauge with respect to the primary constraints and have reduced the problem to the remaining degrees of freedom. They never appeared anymore as independent variables and in the effective, physical Hamiltonian description lapse and shift were derived quantities as well, they were not independent.

\subsection{Subtleties with Lapse and Shift Functions}

In order to compare our framework with SCPT we must deal with the following subtlety:

As mentioned earlier, in the Hamiltonian framework we have ten gravitational configuration and momentum degrees of freedom to begin with. These are $n, n^{a}, q_{a b}$, denoting lapse function, shift vector and three metric, respectively, as well as their conjugate momenta $p, p_{a}, p^{a b}$, respectively. This phase space is subject to four primary constraints $z=p=0, z_{a}=p_{a}=0$, as well as four secondary constraints $c^{\text {tot }}=c^{\text {dust }}+c^{\text {geo }}+c^{\text {matter }}$ and $c_{a}^{\text {tot }}=c_{a}^{\text {dust }}+c_{a}^{\text {geo }}+c_{a}^{\text {matter }}$. These eight constraints are first-class and play a dual role: on the one hand they constrain the phase space to the constraint surface, on the other hand they generate gauge transformations via canonical transformations. The constraint equations can be used to eliminate eight of the momenta while the gauge transformations eliminate eight of the configuration degrees of freedom. Hence the physical or reduced phase space has eight canonical pairs less than the unreduced one. The primary constraints and the gauge transformations generated by them, which consist in arbitrary changes of lapse and shift, completely decouple from the rest of the equations, namely $c^{\text {tot }}, c_{a}^{\text {tot }}$ do not depend on $n, n^{a}, p, p_{a}$, in particular, the gauge transformations generated by $c^{\text {tot }}, c_{a}^{\text {tot }}$ do not affect lapse and shift functions. It is therefore mathematically convenient to simply forget about $p, p_{a}$ and to treat $n, n^{a}$ as Lagrange multipliers which is customary. 
Let us compare this with the situation in the Lagrangian framework. Here we consider only the ten gravitational configuration coordinates, momenta are never introduced. The system is subject to Einstein's equations. Four of them, the temporal - temporal and the temporal - spatial ones are equivalent to the secondary constraints that we also find in the Hamiltonian formalism. Namely, in the Hamiltonian formalism the equations of motion are generated by the primary Hamiltonian

$$
H_{\text {primary }}=\int_{\mathcal{X}} d^{3} x\left(\nu z+\nu^{a} z_{a}+n c^{\text {tot }}+n^{a} c_{a}^{\text {tot }}\right),
$$

where $\nu, \nu^{a}$ are Lagrange multiplier fields. The equation of motion for $q_{a b}$ following from (4.34) enables us to express $p^{a b}$ in terms of $q_{a b}, \dot{q}_{a b}, n, n^{a}$, specifically

$$
p^{a b}=\sqrt{\operatorname{det}(q)}\left(q^{a c} q^{b d}-q^{a b} q^{c d}\right) K_{c d} \quad \text { with } \quad K_{a b}=\frac{1}{2 n}\left(\dot{q}_{a b}-2 D_{(a} n_{b)}\right) .
$$

Here we introduced the extrinsic curvature $K_{a b}$, and $D$ is the torsion free covariant differential compatible with $q_{a b}$. When inserting (4.35) into $c^{\text {geo }}, c_{a}^{\text {geo }}$, one obtains precisely the temporal - temporal and temporal - spatial components of the Einstein tensor, respectively. Thus we can match four of the equations of motion in the Hamiltonian and the Lagrangian equations of motion. The remaining spatial - spatial components of the Einstein equations are obtained in the Hamiltonian formalism by inserting (4.35) into the equations of motion for $\ddot{q}_{a b}$ which also uses the equation of motion for $p^{a b}$ as obtained from (4.34) [19], so that we get an exact match at the level of the equations of motion (one has to use that the spacetime metric is expressed in terms of the configuration coordinates as $\left.g_{t t}=-n^{2}+q_{a b} n^{a} n^{b}, g_{t a}=q_{a b} n^{b}, g_{a b}=q_{a b}\right)$. Now in the Lagrangian formalism the secondary constraints do not generate gauge transformations because one does not have a phase space formulation. Rather one notices that the Lagrangian and thus the Einstein equations are invariant under spacetime diffeomorphisms. Thus one imposes spacetime diffeomorphism invariance as a gauge symmetry. Now we have seen that the temporal - temporal and temporal - spatial components of the Einstein equations do not involve time derivatives of lapse and shift. Thus it is natural to eliminate them via the secondary constraint in terms of $q_{a b}, \dot{q}_{a b}$ and to eliminate four components of $q_{a b}$ by using spacetime diffeomorphism gauge transformations.

We see that in both formalisms we get the same number of physical configuration degrees of freedom, however, the avenue to get there is somewhat different. In particular, there is no counterpart of the gauge symmetry associated with the primary constraints at the Lagrangian level. Furthermore, while the secondary constraints generate spacetime diffeomorphisms on $q_{a b}$ [19], they preserve lapse and shift fields, whereas in the Lagrangian formalism lapse and shift do change under spacetime diffeomorphisms. Thus, there is a mismatch in the number and action of the gauge transformations of the Lagrangian and the Hamiltonian formalism at the level of the unreduced configuration space. It is clear that the physical degrees of freedom that both formalisms produce are the same, since both formalisms tell us that one should extract the spacetime diffeomorphism invariant information contained in $q_{a b}$ (as far as the gravitational degrees of freedom are concerned). However, before using the secondary constraints, the diffeomorphism invariant quantities that one constructs in the Hamiltonian theory do not involve lapse and shift because they are invariant under the secondary constraints, while in the Lagrangian formalism one has to use them. This has the consequence that in the Lagrangian formalism one has in principle six rather than two physical configuration degrees of freedom whereas in the Hamiltonian formalism one has only two. In the Lagrangian formalism, four of them are eliminated via the secondary constraints and thus, at the end of the day, one gets an exact match at the level of the physical degrees of freedom. But before doing that it is somewhat difficult to compare the gauge invariant degrees of freedom in the two formalisms. The reason for why that happens lies deeper and has to do with the question whether Lagrangian (Noether) symmetries have canonical generators in the Hamiltonian formalism. This is analysed in all detail in [20].

Fortunately, there is an elegant solution. The idea [20] is to add, in the Hamiltonian formalism, to the secondary constraints a linear combination of the primary constraints with carefully chosen coefficients such that the new constraints generate spacetime diffeomorphisms on the full phase space, including lapse and 
shift. This leads to an equivalent set of constraints which leads to the same final set of physical degrees of freedom but which has, after reducing with respect to the new secondary constraints only, the same gauge-invariant degrees of freedom as in the Lagrangian formalism. Then afterwards one must impose the secondary constraints in the Lagrangian formalism and reduce with respect to the primary constraints in the Hamiltonian formalism in order to arrive at the true degrees of freedom. We will now sketch how this is done as we will need this in the subsequent subsections.

Fortunately, we do not need all the machinery developed in [20, but can rather develop the required formulae by basic methods. The starting point is to recall [19] that for arbitrary test functions $b, b^{a}$ we have

$$
\left\{c^{\text {tot }}(b)+\vec{c}^{\text {tot }}(\vec{b}), q_{\mu \nu}(x)\right\}=\mathcal{L}_{u} q_{\mu \nu}(x) \quad \text { where } \quad u^{\mu}=b n^{\mu}+X_{, a}^{\mu} b^{a}
$$

with $c^{\text {tot }}(f)=\int_{\mathcal{X}} d^{3} x b(x) c^{\text {tot }}(x)$ and $\vec{c}^{\text {tot }}(\vec{b})=\int_{\mathcal{X}} d^{3} x b^{a}(x) c_{a}^{\text {tot }}(x)$. Here $\mathcal{L}_{u}$ denotes the spacetime Lie derivative with respect to the vector field $u$ and $q_{\mu \nu}=g_{\mu \nu}+n_{\mu} n_{\nu}$ is the three metric on the leaves $\mathcal{X}_{t}$ of the foliation, with unit normal $n^{\mu}$, parametrised by the one parameter family of embeddings $Y_{t}=Y(t,$.$) :$ $\mathcal{X} \rightarrow \mathcal{X}_{t}$. In deriving (4.36) the equations of motion have been used and thus we can say that the secondary constraints generate infinitesimal spacetime diffeomorphisms parametrised by $u$. Notice that any vector field can be split into components tangential and orthogonal to the foliation so that this is no loss of generality.

We now would like to add to $c^{\text {tot }}(b)$ and $\vec{c}^{\text {tot }}(\vec{b})$, respectively, a term linear in $p$ and $p_{a}$, respectively, with smearing functions as coefficients that are linear in both $b, b^{a}$ such the extended constraints also generate infinitesimal spacetime diffeomorphisms on lapse and shift functions parametrised by the above vector field $u$. In order to determine those coefficients, we first need to determine the transformation behaviour of lapse and shift functions under infinitesimal spacetime diffeomorphisms. Starting from the identities $g_{t t}=-n^{2}+q_{a b} n^{a} n^{b}, g_{t a}=q_{a b} n^{b}, g_{a b}=q_{a b}$, we can solve for lapse and shift in terms of spacetime metric components

$$
n^{a}=g^{a b} g_{t b}, n^{2}=-g_{t t}+g^{a b} g_{t a} g_{t b},
$$

where $g^{a b}$ is the inverse of the spatial metric $g_{a b}$. Now using that the change of the metric under infinitesimal diffeomorphisms is given by the Lie derivative

$$
\delta_{u} g_{\mu \nu}=\left(\mathcal{L}_{v} g\right)_{\mu \nu}=u^{\rho} g_{\mu \nu, \rho}+2 g_{\rho(\mu} u_{, \nu)}^{\rho},
$$

we can work out the infinitesimal transformation of lapse and shift. The result is, in the frame where $X^{0}=t$ and $X^{a}=x^{a}$ (frame adapted to the embedding),

$$
\begin{aligned}
\delta_{u} n^{a}= & u^{b} n_{, b}^{a}-n^{b} u_{, b}^{a}+u_{, t}^{a}+\left(n^{a} u^{t}\right)_{, t}-\left(n^{2} q^{a b}+n^{a} n^{b}\right) u_{, b}^{t} \\
2 n \delta_{u} n= & -n^{a} n^{b}\left(u^{c} g_{a b, c}+2 u_{,(a}^{c} g_{b) c}+u^{t} g_{a b, t}+2 n_{a} u_{, b}^{t}\right) \\
& +2 n^{a}\left(u^{t} g_{a b, t}+u_{, t}^{t} g_{a t}+g_{t t} u_{, a}^{t}+u^{b} g_{a t, b}+g_{a b} u_{, t}^{b}+u_{, a}^{b} g_{b c} n^{c}\right) \\
& -\left(g_{t t, t} u^{t}+2 g_{t t} u_{, t}^{t}+g_{t t, a}+2 n_{a} u_{, t}^{a}\right) .
\end{aligned}
$$

Here we have used the explicit form of (4.38). Formula (4.39) is rather complicated. Worse than that, it contains time derivatives of $n, n^{a}$. We cannot use the equations of motion in order to replace those time derivatives by the momenta $p, p_{a}$ because the very reason for the appearance of the primary constraints $p=p_{a}$ is that the Legendre transform is singular, as the Einstein Hilbert action does not contain time derivatives of lapse and shift. This is different for time derivatives of $q_{a b}$. Hence, in order to write (4.39) in terms of canonical coordinates, we must get rid of the time derivatives of lapse and shift functions. Miraculously, the equations (4.39) simplify drastically when we split $u$ into normal and tangential pieces, $u^{\mu}=b n^{\mu}+b^{a} Y_{, a}^{\mu}$. Noticing that in the chosen frame we have from $g_{\mu \nu} n^{\mu} n^{\nu}=-1, Y_{, a}^{\mu} g_{\mu \nu} n^{\nu}=0$ that $n^{\mu=t}=1 / n, n^{\mu=a}=-n^{a} / n$, as well as $n_{t}=-n, n_{a}=0$, we find

$$
u^{t}=\frac{b}{n}, u^{a}=b^{a}-b \frac{n^{a}}{n} .
$$


Substituting (4.40) into (4.39), almost everything cancels due to the explicit lapse and shift dependence of the vector field $u$ and yields after some calculus

$$
\begin{aligned}
\delta_{u} n^{a} & =\left(\mathcal{L}_{\vec{b}} \vec{n}\right)^{a}+q^{a b}\left(b n_{, b}-n b_{, b}\right)+b_{, t}^{a} \\
2 n \delta_{u} n & =2 n\left(b_{, t}+b^{a} n_{, a}-n^{a} b_{, a}\right) .
\end{aligned}
$$

The time derivatives of lapse and shift have disappeared as desired. The required extension of $c^{\text {tot }}(b)+\vec{c}^{\text {tot }}(\vec{b})$ that generates spacetime diffeomorphisms on all ten components of the spacetime metric and not only its spatial - spatial components is therefore given by

$$
\int_{\mathcal{X}} d^{3} x\left(p_{a}\left[\left(\mathcal{L}_{\vec{b}} \vec{n}\right)^{a}+q^{a b}\left(b n_{, b}-n b_{, b}\right)+b_{, t}^{a}\right]+p\left[b_{, t}+b^{a} n_{, a}-n^{a} b_{, a}\right]\right) .
$$

The coefficients appearing in (4.42) are no accident, but directly derive from the Dirac algebra of secondary constraints as shown explicitly in [20]. It follows that the primary constraints have to be there in order to make the formalism manifestly spacetime covariant. We observe that when choosing $b=n, b^{a}=n^{a}$ the modified expression $c^{\text {tot }}(f)+\vec{c}^{\text {tot }}(\vec{f})+(4.42)$ turns precisely into the primary Hamiltonian (4.34), because the equation of motion for lapse and shift can be used to eliminate $\nu=b, \nu^{a}=b^{a}$. Thus we may say that for those special smearing fields the generator of spacetime diffeomorphisms is nothing but the primary Hamiltonian. Thus, the extended generator of spacetime diffeomorphisms is nothing but the natural extension of the primary Hamiltonian to arbitrary test functions different from $b=n, b^{a}=n^{a}$.

Performing integrations by parts (using suitable fall-off properties of $b, b^{a}$ ), we collect the coefficients of $b^{a}$ and $b$ in (4.42) (but not of $\dot{b}^{a}, \dot{b}$ ), which give the lapse and shift (LS) contributions to the spatial diffeomorphism and Hamiltonian constrain,t respectively:

$$
\begin{aligned}
c_{a}^{L S} & =p n_{, a}+p_{b} n_{, a}^{b}+\left[p_{b} n^{b}\right]_{, a} \\
c^{L S} & =\left[q^{a b} p_{b} n+n^{a} p\right]_{, a}+q^{a b} p_{b} n_{, a} .
\end{aligned}
$$

The first equation tells us that $n, n^{a}$ transform as scalar and vector, respectively, under spatial diffeomorphims, while $p, p_{a}$ are correspondingly scalar and covector densities, respectively. For this reason all the derivatives in the second equation in (4.43) can be replaced by covariant ones.

Since we have extended the phase space by lapse and shift, we now must include the contributions (4.43) into the secondary constraints which thus read

$$
\begin{aligned}
c^{\mathrm{tot} \prime} & =\left[c^{\mathrm{LS}}+c^{\mathrm{geo}}+c^{\text {matter }}\right]+c^{\mathrm{dust}}=: c^{\prime}+c^{\mathrm{dust}} \\
c_{a}^{\mathrm{tot} \prime} & =\left[c_{a}^{\mathrm{LS}}+c_{a}^{\mathrm{geo}}+c_{a}^{\mathrm{matter}}\right]+c_{a}^{\mathrm{dust}}=: c_{a}^{\prime}+c_{a}^{\text {dust }} .
\end{aligned}
$$

They have to be used in our formula for gauge-invariant completions with respect to the secondary spatial diffeomorphism and Hamiltonian constraints, respectively. Now, as before, we solve (4.44) for the dust momenta and obtain

$$
\begin{aligned}
& \tilde{c}^{\text {tot' }}=P+\sqrt{\left[c^{\prime}\right]^{2}+q^{a b} c_{a}^{\prime} c_{b}^{\prime}}=: P+h^{\prime} \\
& \tilde{c}_{a}^{\text {tot }}=P_{j}+S_{j}^{a}\left(-h^{\prime} T_{, a}+c_{a}^{\prime}\right)
\end{aligned}
$$

which is completely analogous to the formalism in which the primary constraints have been reduced already.

The $\dot{b}$ and $\dot{b}^{a}$ terms in (4.42) cannot be taken care of in $c^{L S}, c_{a}^{L S}$. In principle, we have to consider $\nu^{0}:=\dot{b}, \nu^{a}:=\dot{b}^{a}$ as independent test functions. Therefore, the $\dot{b} p+\dot{b}^{a} p_{a}=\nu^{\mu} z_{\mu}$ contributions in (4.42) have to be attributed to the primary constraints $z_{\mu}=p_{\mu}$, that is, $z=p, z_{a}=p_{a}$. Since the primary constraints have not yet been reduced, we must also perform the gauge-invariant completion with respect 
to the primary constraints. Notice that since $z, z_{a}, c^{\text {tot }}, c_{a}^{\text {tot }}$ are weakly Poisson commuting, so are the $z, z_{a}, c^{\text {tot' }}, c_{a}^{\text {tot } /}$ because (4.43) is a linear combination of the $z, z_{a}$. Hence the system remains first class. Since $z, z_{a}$ are presented in deparametrised form, we know that the constraints $z, z_{a}, \tilde{c}^{\text {tot }}, \tilde{c}_{a}^{\text {tot }}$ are mutually Poisson commuting. Hence, in addition to what we did in our companion paper, we must supplement the gauge-invariant extension $F$ of some function $f$ with respect to the secondary constraints by gauge-invariant extensions with respect to the primary constraints $z, z_{a}$, for which of course we use as clocks the lapse and shift functions $n, n^{a}$. Specifically, that last extension is given by the formula

$$
O_{F}^{(3)}[\nu]=F+\sum_{n=1}^{\infty} \frac{1}{n !} \int_{\mathcal{X}} d^{3} x_{1} . . \int_{\mathcal{X}} d^{3} x_{n}\left(\nu^{\mu_{1}}-n^{\mu_{1}}\right)\left(x_{1}\right) . .\left(\nu^{\mu_{n}}-n^{\mu_{n}}\right)\left(x_{n}\right) . .\left\{z_{\mu_{1}}\left(x_{1}\right), . .,\left\{z_{\mu_{n}}\left(x_{n}\right), F\right\} . .\right\}
$$

with arbitrary functions $\nu^{\mu}, \mu=0,1,2,3$ and we have set $n^{0}:=n, n^{\mu=a}:=n^{a}, a=1,2,3$. This operation leaves functions independent of $n, n^{a}$ unaffected, of course, and, more generally, replaces any $n^{\mu}$ by $\nu^{\mu}$, in accordance with the fact that $n, n^{a}$ are pure gauge with respect to the primary constraints.

Notice that the gauge-invariant projection $O^{(3)}$ has to be performed after the operations $O^{(1)}, O^{(2)}$ of our companion paper, because $p, p_{a}$ Poisson commute with $T, S^{j}$, but $n, n^{a}$ do not Poisson commute with $\tilde{c}_{a}^{\text {tot }}, \tilde{c}_{a}^{\text {tot } \prime}$. Since in the complete projection the constraints never act on the $\tau-T, \sigma^{j}-S^{j}, \nu-n, \nu^{a}-n^{a}$ powers, (4.46) has to applied in this order.

With this machinery at our disposal we can now continue our comparison with the SCPT framework.

\subsection{Comparison with the SCPT framework for Lapse and Shift}

As discussed in the last section, for the purpose of comparing with the SCPT framework, we have to use the extended Hamiltonian formalism. Thus we now must use the constraints shown in equation (4.44), as well as the additional projection in equation (4.45). All that we have said before remains valid because on the constraint surface defined by the primary constraints the constraints (4.44) coincide with the old ones. Let us write the perturbed expansion to linear order of a complete gauge-invariant quantity in the extended phase space as

$$
\delta O_{f}=\delta f-\int_{\mathcal{X}} d^{3} y\left(\delta T(y) \overline{\left\{\tilde{c}^{\mathrm{tot} \prime}(y), f\right\}}+\delta S^{j}(y) \overline{\left\{\tilde{c}_{j}^{\mathrm{tot} \prime}(y), f\right\}}+\delta n(y) \overline{\{z(y), f\}}+\delta n^{a}(y) \overline{\left\{z_{a}(y), f\right\}}\right)
$$

where

$$
\delta T=T-\tau, \quad \delta S^{j}=S^{j}-\sigma^{j}, \quad \delta n=n-\nu, \quad \text { and } \quad \delta n^{a}=n^{a}-\nu^{a} .
$$

Evaluating (4.47) for the functions $f=n(x), n^{a}(x)$ yields

$$
\begin{aligned}
\delta \tilde{n}(x):=\delta O_{n(x)} & =\left[\delta n-\overline{S_{j}^{a}} \delta S^{j} \overline{n_{, a}}+\overline{n^{a}}[\delta T]_{, a}-\delta n\right](x) \\
& =0 \\
\delta \tilde{n}^{a}(x):=\delta O_{n^{a}(x)} & =\left[\delta n^{a}-\overline{S_{j}^{b}} \delta S^{j} \overline{n_{, b}^{a}}+\overline{n^{b}}\left[\overline{S_{j}^{a}} \delta S^{j}\right]_{, b}-\overline{q^{a b}}\left(\delta T \bar{n}_{, b}-\bar{n}[\delta T]_{, b}\right)-\delta n^{a}\right](x) \\
& =\frac{\delta^{a b}}{a^{2}}[\delta T]_{, b}(x)
\end{aligned}
$$

where we used $\bar{n}=1, \overline{n^{a}}=0$. We want to compare $\tilde{n}$ and $\tilde{n}_{a}$ with $\delta \tilde{g}_{00}$ and $\delta \tilde{g}_{0 a}$, respectively, in equation (4.12). From equation (4.27) we can read off

$$
\delta \tilde{g}_{00}=-2 a^{2} \delta \tilde{n} \quad \text { and } \quad \delta \tilde{g}_{0 a}=a^{3} \delta_{a b} \delta \tilde{n}^{b} .
$$


Hence the expressions for $\delta \tilde{g}_{00}$ and $\delta \tilde{g}_{0 a}$ in equation (4.12) are equivalent to

$$
\delta \tilde{n}=\delta n-[\delta \dot{T}] \quad \text { and } \quad \delta \tilde{n}^{a}=\delta n^{a}-\left[\bar{S}_{j}^{a} \delta \dot{S}^{j}-\frac{\delta^{a b}}{a^{2}}[\delta T]_{, b}\right],
$$

where we used that $\dot{S}_{j}^{a}=0$. Comparing the Lagrangian result (4.52) with the Hamiltonian result (4.49), we do not seem to obtain a match. The reason is the appearance of the time derivatives of $\delta T$ and $\delta S^{j}$. On the other hand, notice that with the identifications $b=\delta T$ and $b^{a}=\bar{S}_{j}^{a} \delta S^{j}$ the square bracket terms on the right hand side of (4.52) precisely cancel the spacetime diffeomorphism transformation derived in equation (4.41) via Hamiltonian methods. The difference is that the fields $b, b^{a}$ in equation (4.41) were smearing fields, while here $\delta T$ and $\delta \bar{S}_{j}^{a} \delta S^{j}$ are phase space dependent functions. The only way in which the Hamiltonian formalism can produce time derivatives of the canonical fields is via the equations of motion. Now the equations of motion in the Hamiltonian formalism which lead to an exact match with the Euler Lagrange equations are with respect to the primary Hamiltonian (4.34). We find

$$
\begin{aligned}
\dot{T}(x) & =\left\{H_{\text {primary }}, T(x)\right\}=\left[n^{a} T_{, a}-n \frac{P}{\sqrt{P^{2}+q^{a b} c_{a}^{\prime} c_{b}^{\prime}}}\right](x) \\
\dot{S}^{j}(x) & =\left\{H_{\text {primary }}, T(x)\right\}=\left[n^{a} S_{, a}^{j}\right](x) .
\end{aligned}
$$

Perturbing equation (4.53) around the FRW background and using $\overline{c_{a}^{\prime}}=\bar{T}_{, a}=0$, as well as $\dot{\bar{S}}_{a}^{j}=0$, we find

$$
\delta \dot{T}=\delta n \quad \text { and } \quad \overline{S_{j}^{a}} \delta \dot{S}^{j}=\delta n^{a} .
$$

Equation (4.54) is evidently the missing link to bring (4.49) and (4.52) to an exact match.

By construction, the full, non-perturbative expression for $O_{n^{a}(x)}$ and $O_{n(x)}$ will be expressed in terms of the fundamental seven physical degrees of freedom corresponding to $q_{a b}, \xi$ and their conjugate momenta. Hence in the Hamiltonian framework (4.52) are of no further interest. In the Lagrangian formalism, the secondary constraints fulfill the task to express those equations in terms of the other degrees of freedom. Hence in both frameworks we end up with seven gauge-invariant degrees of freedom.

Summarising, we showed that, using the four dust fields $\delta T, \delta S^{k}$, we are able to construct (up to linear order) gauge-invariant quantities along the lines of SCPT. We obtain 10 gauge-invariant components $\delta \widetilde{g}_{\mu \nu}$ and one for the matter scalar field $\delta \widetilde{\xi}$. Out of these 11 degrees of freedom only 7 are physically relevant, because apart from the non-physical dust degrees of freedom the system is reduced by another four degrees of freedom due to the four primary constraints of General Relativity. This leads to a reduction from the 15 configuration degrees of freedom of the system "gravity + scalar field + dust" down to 7 true degrees of freedom. These seven degrees of freedom reside in the perturbations of the three metric $\delta \widetilde{g}_{a b}=\delta \widetilde{q}_{a b}$ and the scalar field $\delta \widetilde{\xi}$. Finally, we expanded the (manifestly) gauge-invariant observables constructed by the method introduced in [1] up to linear order in the perturbations and compared them with $\delta \widetilde{g}_{\mu \nu}$ and $\delta \widetilde{\xi}$. It was shown that these two ways of constructing (up to linear order) gauge-invariant quantities lead to exactly the same results. Four of those variables are redundant in both formalisms. Thus, for the physical degrees of freedom we get an exact match. In contrast to section 3 where SCPT without dust was considered, we also have an agreement on the number of physical degrees of freedom here, 7 for SCPT and 7 in our framework. However, as was discussed in section 3 , the equations of motion derived for the 7 degrees of freedom show that 4 of these degrees of freedom freeze out in the late universe, so that the physics is mainly governed by the 3 degrees of freedom used in SCPT.

\section{$5 \quad$ Summary and Conclusions}

In this paper we applied a new framework for general relativistic perturbation theory, developed in our companion paper [1, to the important case of cosmological perturbations. The central feature of our new 
approach is the use of a dynamically coupled observer medium, given by pressureless dust. This allows for a complete deparametrisation of the physical system of interest, that is gravity coupled to whatever matter one wishes to include. As a result, the usual gauge freedom of general relativity is eliminated and true observables are obtained, together with a true time evolution generated by a physical Hamiltonian. By specializing our general framework to the case of an FRW background spacetime, we developed a manifestly gauge-invariant cosmological perturbation theory. As the quantities that are perturbed, namely the threemetric and all the (non-dust) matter fields, are already fully gauge-invariant by construction, the familiar problems with gauge freedom, that had been troublesome for standard cosmological perturbation theory (SCPT) for a long time, never arise here. In particular, it is straightforward, though no doubt involved, to derive perturbed equations of motions to arbitrarily high order.

In this paper, we limited ourselves to investigating the linear order. We found that up to a small correction term, our formalism reproduces the known SCPT equations. The correction term is an imprint of the dust system. On physical grounds, this could have been anticipated right from the start, as the observer dust adds to the overall energy momentum balance. Our framework thus illustrates the fact that including a realistic, that is non-idealized, observer in the description of a physical system will always disturb the original system to some extent. As this correction turned out to be inversely proportional to the scale factor, however, it decays away very quickly in the early universe. Consequently, the equations of motion coming out of our framework are well within an acceptable range of the standard cosmological model results.

There are many routes for further investigations that one can take from here. Since the issue of gauge invariance has been entirely settled to all orders in our approach to perturbation theory, the obvious next step is to calculate the equations of motion for higher order perturbations, in particular for the second order [18. As mentioned in the introduction, the latter is a topic that currently attracts a considerably amount of attention, due to its connection with the issue of non-Gaussianity of cosmological perturbations. This should also help to settle the stability issue of linear perturbation theory [23]. Another interesting project is to investigate the spherically symmetric sector of the theory and to determine whether there are modifications of Newton's law at large distances. In fact, it might not be totally surprising if there were modifications because the dust is a perfect candidate for a WIMP, one of the candidates for dark matter, since it only interacts gravitationally.

\section{Acknowledgements}

K.G. thanks the Perimeter Institute for Theoretical Physics for hospitality and financial support. Research performed at Perimeter Institute for Theoretical Physics is supported in part by the Government of Canada through NSERC and by the Province of Ontario through MRI. O.W. was partially supported by the Province of Ontario through an ERA award, ER06-02-298.

\section{A Review of Standard Cosmological Perturbation Theory}

For the benefit of the reader we collect here the most important formulae of the SCPT formalism, adapted to our notation. In particular, notice that we are using the relativist's signature $(-,+,+,+)$, which is opposite to the one used by cosmologists.

\section{A.1 Curvature and Energy Momentum Tensor}

Our convention for the curvature tensor is

$$
R_{\mu \nu \rho}{ }^{\sigma} \omega_{\sigma}:=\left[\nabla_{\mu}, \nabla_{\nu}\right] \omega_{\rho} .
$$


Its explicit expression in terms of the Christoffel symbols (we use the notation $(.)_{, \mu}:=\partial(.) / \partial x^{\mu}$ )

$$
\Gamma_{\mu \nu}^{\sigma}=g^{\sigma \rho} \Gamma_{\rho \mu \nu}, \quad \Gamma_{\rho \mu \nu}=\frac{1}{2}\left(g_{\rho \mu, \nu}+g_{\rho \nu, \mu}-g_{\mu \nu, \rho}\right),
$$

corresponding to the torsion free covariant differential $\nabla$ compatible with a general metric $g_{\mu \nu}$ with inverse $g^{\mu \nu}$, is

$$
R_{\mu \nu \rho}^{\sigma}=2\left(-\partial_{[\mu} \Gamma_{\nu] \rho}^{\sigma}+\Gamma_{\rho[\mu}^{\lambda} \Gamma_{\nu] \lambda}^{\sigma}\right)
$$

From this we infer the Ricci tensor, Ricci scalar and Einstein tensor, respectively:

$$
R_{\mu \nu}:=R_{\mu \rho \nu}^{\rho}, R:=g^{\mu \nu} R_{\mu \nu}, G_{\mu \nu}=R_{\mu \nu}-\frac{1}{2} R g_{\mu \nu} .
$$

The energy momentum tensor for bosonic matter is given by

$$
T_{\mu \nu}:=-\frac{2}{\sqrt{|\operatorname{det}(g)|}} \frac{\delta S_{\text {matter }}}{\delta g^{\mu \nu}}
$$

which, for a minimally coupled scalar field $\zeta$ with action

$$
S_{\text {matter }}=-\frac{1}{2 \lambda} \int_{M} d^{4} X \sqrt{|\operatorname{det}(g)|}\left(g^{\mu \nu}\left(\nabla_{\mu} \zeta\right)\left(\nabla_{\nu} \zeta\right)+v(\zeta)\right)
$$

(where $v$ is its potential), becomes

$$
\lambda T_{\mu \nu}=\left[\nabla_{\mu} \zeta\right]\left[\nabla_{\nu} \zeta\right]-\frac{1}{2} g_{\mu \nu}\left(g^{\rho \sigma}\left(\nabla_{\rho} \zeta\right)\left(\nabla_{\sigma} \zeta\right)+v(\zeta)\right) .
$$

The Euler-Lagrange equations for the gravity and scalar matter system (including a cosmological term)

$$
S_{\text {geo }}=\frac{1}{\kappa} \int_{M} d^{4} X \sqrt{|\operatorname{det}(g)|}(R-2 \Lambda)
$$

are the Einstein equations

$$
G_{\mu \nu}+\Lambda g_{\mu \nu}=\frac{\kappa}{2} T_{\mu \nu}
$$

and the matter field equation

$$
g^{\mu \nu} \nabla_{\mu} \nabla_{\nu} \zeta=\frac{1}{2} v^{\prime}(\zeta)
$$

\section{A.2 FRW Background}

The spatial constant curvature $k=0$ FRW line element with scale factor $a(t)$ reads

$$
d s^{2}=-d t^{2}+a(t)^{2} \delta_{a b} d x^{a} d x^{b}=a\left(x^{0}\right)^{2} \eta_{\mu \nu} d x^{\mu} d x^{\nu}
$$

where we have switched to conformal time $d x^{0}:=d \eta:=d t / a(t)$ and $\eta_{\mu \nu}$ denotes the Minkowski metric. In the second step we should actually use a different symbol $\tilde{a}\left(x^{0}\right):=a\left(t\left(x^{0}\right)\right)$, but we will slightly abuse the notation as is customary.

All quantities that refer to a background metric will carry a bar. For the FRW background metric $\bar{g}_{\mu \nu}=a^{2} \eta_{\mu \nu}$ a short computation reveals

$$
\begin{aligned}
\bar{\Gamma}_{00}^{0} & =\frac{a^{\prime}}{a}=: \mathcal{H} \\
\bar{\Gamma}_{0 a}^{0} & =0 \\
\bar{\Gamma}_{a b}^{0} & =\mathcal{H} \delta_{a b} \\
\bar{\Gamma}_{00}^{a} & =0 \\
\bar{\Gamma}_{0 b}^{a} & =\mathcal{H} \delta_{b}^{a} \\
\bar{\Gamma}_{b c}^{a} & =0 .
\end{aligned}
$$


Here and in what follows a prime denotes derivation with respect to conformal time $x^{0}=\eta$, rather than cosmological time $t$, and we have introduced the Hubble function $\mathcal{H}=a^{\prime} / a$. It is useful to note that (A.12) implies that

$$
\bar{\Gamma}_{\nu \mu}^{\nu}=4 \mathcal{H} \delta_{\mu 0}
$$

Carefully using the definitions, we find again after a short calculation

$$
\begin{aligned}
\bar{R}_{00} & =-3 \mathcal{H}^{\prime} \\
\bar{R}_{0 a} & =0 \\
\bar{R}_{a b} & =\left(\mathcal{H}^{\prime}+2 \mathcal{H}^{2}\right) \delta_{a b} \\
\bar{R} & =\frac{6}{a^{2}}\left(\mathcal{H}^{\prime}+\mathcal{H}^{2}\right) \\
\bar{G}_{00} & =3 \mathcal{H}^{2} \\
\bar{G}_{0 a} & =0 \\
\bar{G}_{a b} & =-\left(2 \mathcal{H}^{\prime}+\mathcal{H}^{2}\right) \delta_{a b} .
\end{aligned}
$$

The non-vanishing components of the energy momentum tensor are

$$
\begin{aligned}
\lambda \bar{T}_{00} & =\left(\bar{\zeta}^{\prime}\right)^{2}+\frac{1}{2} a^{2}\left(-\frac{1}{a^{2}}\left(\bar{\zeta}^{\prime}\right)^{2}+v(\bar{\zeta})\right)=\frac{1}{2}\left(\left(\bar{\zeta}^{\prime}\right)^{2}+a^{2} v(\bar{\zeta})\right)=: a^{2} \lambda \bar{\rho} \\
\lambda \bar{T}_{a b} & =\frac{1}{2}\left(\left(\bar{\zeta}^{\prime}\right)^{2}-a^{2} v(\bar{\zeta})\right) \delta_{a b}=: a^{2} \lambda \delta_{a b} \bar{p},
\end{aligned}
$$

where we have introduced background energy density $\bar{\rho}$ and background pressure $\bar{p}$, respectively. The matter field equation becomes

$$
\frac{1}{\sqrt{|\operatorname{det}(\bar{g})|}} \partial_{\mu} \bar{g}^{\mu \nu} \sqrt{|\operatorname{det}(\bar{g})|} \partial_{\nu} \bar{\zeta}=-\frac{1}{a^{4}}\left(a^{2} \bar{\zeta}^{\prime}\right)^{\prime}=-\frac{1}{a^{2}}\left(2 \mathcal{H} \bar{\zeta}^{\prime}+\bar{\zeta}^{\prime \prime}\right)=\frac{1}{2} v^{\prime}(\bar{\zeta})
$$

\section{A.3 Linear Perturbations}

We consider perturbations $\delta g_{\mu \nu}:=g_{\mu \nu}-\bar{g}_{\mu \nu}$ and $\delta \zeta=\zeta-\bar{\zeta}$. Any function $F=F(g, \zeta)$ of the metric $g$ and the scalar field $\zeta$ is expanded to linear order in $\delta g, \delta \zeta$, that is, $\delta F$ will denote the linear order term in the Taylor expansion of $F(g, \zeta)-F(\bar{g}, \bar{\zeta})$. For instance,

$$
\begin{aligned}
\delta \Gamma_{\mu \nu}^{\sigma} & =\delta g^{\sigma \rho} \bar{\Gamma}_{\rho \mu \nu}+\bar{g}^{\sigma \rho} \delta \Gamma_{\rho \mu \nu} \\
& =-\bar{g}^{\sigma \lambda} \delta g_{\lambda \tau} \bar{g}^{\tau \rho} \bar{\Gamma}_{\rho \mu \nu}+\bar{g}^{\sigma \rho} \delta \Gamma_{\rho \mu \nu} \\
& =-\bar{g}^{\sigma \lambda} \delta g_{\lambda \rho} \bar{\Gamma}_{\mu \nu}^{\rho}+\bar{g}^{\sigma \rho} \frac{1}{2}\left(\delta g_{\rho \mu, \nu}+\delta g_{\rho \nu, \mu}-\delta g_{\mu \nu, \rho}\right) .
\end{aligned}
$$

For the FRW background this yields

$$
\delta \Gamma_{\mu \nu}^{\sigma}=\frac{1}{a^{2}}\left[-\eta^{\sigma \lambda} \delta g_{\lambda \rho} \bar{\Gamma}_{\mu \nu}^{\rho}+\eta^{\sigma \rho} \frac{1}{2}\left(\delta g_{\rho \mu, \nu}+\delta g_{\rho \nu, \mu}-\delta g_{\mu \nu, \rho}\right)\right]
$$


Inserting (A.18), we find explicitly

$$
\begin{aligned}
\delta \Gamma_{00}^{0} & =-\left(\frac{1}{2 a^{2}} \delta g_{00}\right)^{\prime} \\
\delta \Gamma_{0 a}^{0} & =\frac{1}{2 a^{2}}\left[2 \mathcal{H} \delta g_{0 a}-\delta g_{00, a}\right] \\
\delta \Gamma_{a b}^{0} & =\frac{1}{2 a^{2}}\left[2 \mathcal{H} \delta g_{00} \delta_{a b}-\left(2 \delta g_{0(a, b)}-\delta g_{a b}^{\prime}\right)\right] \\
\delta \Gamma_{00}^{a} & =\frac{1}{2 a^{2}}\left(-2 \mathcal{H} \delta g_{0 a}+2 \delta g_{0 a}^{\prime}-\delta g_{00, a}\right) \\
\delta \Gamma_{b 0}^{a} & =\frac{1}{2 a^{2}}\left(-2 \mathcal{H} \delta g_{a b}+\delta g_{a b}^{\prime}+2 \delta g_{0[a, b]}\right) \\
\delta \Gamma_{b c}^{a} & =\frac{1}{a^{2}}\left(-\mathcal{H} \delta g_{0 a} \delta_{b c}+2 \delta g_{a(b, c)}^{\prime}-\delta g_{b c, a}\right) .
\end{aligned}
$$

It is useful to note that (A.19) implies

$$
\delta \Gamma_{\nu \mu}^{\nu}=\left[\frac{1}{2 a^{2}}\left(\delta^{b c} \delta g_{b c}-\delta g_{00}\right)\right]_{, \mu} .
$$

Now we use (A.2) and (A.3) to find

$$
\delta R_{\mu \nu}=-2 \partial_{[\mu} \delta \Gamma_{\rho] \nu}^{\rho}+\bar{\Gamma}_{\rho \sigma}^{\rho} \delta \Gamma_{\mu \nu}^{\sigma}+\bar{\Gamma}_{\mu \nu}^{\sigma} \delta \Gamma_{\rho \sigma}^{\rho}-2 \bar{\Gamma}_{\sigma(\mu}^{\rho} \delta \Gamma_{\nu) \rho}^{\sigma} .
$$

Specialising (A.21) to the temporal - temporal, temporal - spatial and spatial - spatial components, respectively, yields

$$
\begin{aligned}
\delta R_{00}= & \left(-\frac{1}{2 a^{2}} \delta g_{c d} \delta^{c d}\right)^{\prime \prime}+\frac{1}{a}\left(\frac{\delta^{c d} \delta g_{0 c, d}}{a}\right)^{\prime}-\frac{1}{2 a^{2}} \Delta \delta g_{00}-\mathcal{H}\left(\frac{1}{2 a^{2}} \delta g_{c d} \delta^{c d}\right)^{\prime}-3 \mathcal{H}\left(\frac{1}{2 a^{2}} \delta g_{00}\right)^{\prime} \\
\delta R_{0 a}= & \left(-\frac{1}{2 a^{2}} \delta g_{c d, a} \delta^{c d}\right)^{\prime}+\left(\frac{\mathcal{H}}{2 a^{2}} \delta g_{0 a}\right)^{\prime}+\left(\frac{1}{2 a^{2}} \delta^{c d} \delta g_{a c, d}\right)^{\prime}+\frac{1}{2 a^{2}}\left(\delta^{c d} \delta g_{0 c, d a}-\Delta \delta g_{0 a}\right) \\
& +\frac{\mathcal{H}}{a^{2}}\left(4 \mathcal{H} \delta g_{0 a}-\delta g_{00, a}-\delta g_{0 a}^{\prime}\right) \\
\delta R_{a b}= & -\frac{1}{2 a^{2}}\left[\delta^{c d} \delta g_{c d}-\delta g_{00}\right]_{, a b}+\left[\frac{\mathcal{H}}{a^{2}} \delta g_{00} \delta_{a b}-\frac{1}{2 a^{2}}\left(2 \delta g_{0(a, b)}-\delta g_{a b}^{\prime}\right)\right]^{\prime} \\
& +2 \mathcal{H}\left[\frac{\mathcal{H}}{a^{2}} \delta g_{00} \delta_{a b}-\frac{1}{2 a^{2}}\left(2 \delta g_{0(a, b)}-\delta g_{a b}^{\prime}\right)\right]+\frac{1}{a^{2}}\left(-\mathcal{H} \delta^{c d} \delta_{a b} \delta g_{0 c, d}+\delta^{c d} \delta \Gamma_{c a b, d}\right) \\
& -2 \mathcal{H}\left(\frac{1}{2 a^{2}} \delta g_{a b}\right)^{\prime}+\mathcal{H} \delta_{a b}\left(\frac{1}{2 a^{2}}\left(\delta^{c d} \delta g_{c d}-\delta g_{00}\right)\right)^{\prime} .
\end{aligned}
$$

Here $\Delta=\delta^{a b} \partial_{a} \partial_{b}$ denotes the flat background Laplacian and $2 \delta \Gamma_{c a b}=2 \delta g_{c(a, b)}-\delta g_{a b, c}$. This implies

$$
\begin{aligned}
\delta R= & -\bar{g}^{\nu \sigma} \bar{g}^{\mu \rho} \delta g_{\rho \sigma} \bar{R}_{\mu \nu}+\bar{g}^{\mu \nu} \delta R_{\mu \nu} \\
= & -\frac{1}{a^{4}}\left(-3 \mathcal{H}^{\prime} \delta g_{00}+\left(\mathcal{H}^{\prime}+2 \mathcal{H}^{2}\right) \delta^{c d} \delta g_{c d}\right)+\frac{1}{a^{2}}\left(-\delta R_{00}+\delta^{c d} \delta R_{c d}\right) \\
\delta^{c d} \delta R_{c d}= & -\frac{1}{2 a^{2}} \Delta\left(\delta^{c d} \delta g_{c d}-\delta g_{00}\right)+\left(3 \frac{\mathcal{H}}{a^{2}} \delta g_{00}-\frac{\delta^{c d}}{2 a^{2}}\left(2 \delta g_{0(c, d)}-\delta g_{c d}^{\prime}\right)\right)^{\prime}-2 \mathcal{H}\left(\frac{\delta^{c d}}{2 a^{2}} \delta g_{c d}\right)^{\prime} \\
& +\frac{1}{a^{2}}\left(-3 \mathcal{H} \delta^{c d} \delta g_{0 c, d}+\delta^{c d} \delta^{a b} \delta \Gamma_{c a b, d}\right)+2 \mathcal{H}\left(3 \frac{\mathcal{H}}{a^{2}} \delta g_{00}-\frac{\delta^{c d}}{2 a^{2}}\left(2 \delta g_{0(c, d)}-\delta g_{c d}^{\prime}\right)\right) \\
& +3 \mathcal{H}\left(\frac{1}{2 a^{2}}\left(\delta^{c d} \delta g_{c d}-\delta g_{00}\right)\right)^{\prime} .
\end{aligned}
$$


Since

$$
\delta G_{\mu \nu}=\delta R_{\mu \nu}-\frac{\bar{R}}{2} \delta g_{\mu \nu}-\frac{\bar{g}_{\mu \nu}}{2} \delta R
$$

we find

$$
\begin{aligned}
\delta G_{00} & =\frac{1}{2}\left(\delta R_{00}+\delta^{c d} \delta R_{c d}\right)-\frac{1}{a^{2}}\left(\frac{1}{2}\left(\mathcal{H}^{\prime}+\mathcal{H}^{2}\right) \delta^{c d} \delta g_{c d}+3 \mathcal{H}^{2} \delta g_{00}\right) \\
\delta G_{0 a} & =\delta R_{0 a}-\frac{3}{a^{2}}\left(\mathcal{H}^{\prime}+\mathcal{H}^{2}\right) \delta g_{0 a} \\
\delta G_{a b} & =\delta R_{a b}-\frac{3}{a^{2}}\left(\mathcal{H}^{\prime}+\mathcal{H}^{2}\right) \delta g_{a b}-\frac{a^{2}}{2} \delta_{a b}\left[-\frac{1}{a^{4}}\left(-3 \mathcal{H}^{\prime} \delta g_{00}+\left(\mathcal{H}^{\prime}+2 \mathcal{H}^{2}\right) \delta^{c d} \delta g_{c d}\right)+\frac{1}{a^{2}}\left(-\delta R_{00}+\delta^{c d} \delta R_{c d}\right)\right] .
\end{aligned}
$$

Next the perturbation of (A.7) yields after a short computation

$$
\lambda \delta T_{\mu \nu}=2 \zeta^{\prime} \delta_{(\mu}^{0}(\delta \zeta)_{, \nu)}-\frac{1}{2} \lambda \bar{p} \delta g_{\mu \nu}+\frac{1}{2} \eta_{\mu \nu}\left(\frac{1}{a^{2}}\left(\bar{\zeta}^{\prime}\right)^{2} \delta g_{00}+2 \bar{\zeta}^{\prime} \delta \zeta^{\prime}-a^{2} v^{\prime}(\bar{\zeta}) \delta \zeta\right)
$$

or, in components,

$$
\begin{aligned}
\delta T_{00} & =\frac{1}{2 \lambda}\left(2 \bar{\zeta}^{\prime} \delta \zeta^{\prime}+a^{2} v^{\prime}(\bar{\zeta}) \delta \zeta-v(\bar{\zeta}) \delta g_{00}\right) \\
\delta T_{0 a} & =\frac{1}{\lambda}\left(\bar{\zeta}^{\prime} \delta \zeta_{, a}+\lambda \bar{p} \delta g_{0 a}\right) \\
\delta T_{a b} & =\frac{1}{\lambda}\left[\lambda \bar{p} \delta g_{a b}+\frac{1}{2} \delta_{a b}\left(\frac{1}{a^{2}}\left(\bar{\zeta}^{\prime}\right)^{2} \delta g_{00}+2 \bar{\zeta}^{\prime} \delta \zeta^{\prime}-a^{2} v^{\prime}(\bar{\zeta}) \delta \zeta\right)\right] .
\end{aligned}
$$

\section{A.4 Parametrisation of perturbations and linear invariants}

The complicated equations of the previous section can be decomposed into scalar, vector and tensor contributions. As in [9] we introduce four scalar fields $\phi, \psi, B, E$, two transversal (with respect to the flat $3 \mathrm{~d}$ Euclidean background metric) covector fields $S_{a}, F_{a}$ and a symmetric tracefree, transversal tensor $h_{a b}$. Thus $\delta^{a b} F_{a, b}=\delta^{a b} S_{a, b}=\delta^{b c} h_{a b, c}=\delta^{a b} h_{a b}=0$. These fields encode the ten independent components of $\delta g_{\mu \nu}$ as follows: We write the perturbed line element

$$
d s^{2}=a^{2} \eta_{\mu \nu} d x^{\mu} d x^{\nu}+\delta g_{00}\left(d x^{0}\right)^{2}+2 \delta g_{0 a} d x^{0} d x^{a}+\delta g_{a b} d x^{a} d x^{b}
$$

in the form

$$
d s^{2}=a^{2}\left[(-1+2 \phi)\left(d x^{0}\right)^{2}+2\left(S_{a}+B_{, a}\right) d x^{0} d x^{a}+\left((1+2 \psi) \delta_{a b}+2 E_{, a b}+2 F_{(a, b)}+h_{a b}\right) d x^{a} d x^{b}\right]
$$

from which one reads off

$$
\begin{aligned}
\delta g_{00} & =+2 a^{2} \phi \\
\delta g_{0 a}= & +a^{2}\left(S_{a}+B_{, a}\right) \\
\delta g_{0 a}= & +a^{2}\left[2\left(\psi \delta_{a b}+E_{, a b}+F_{(a, b)}\right)+h_{a b}\right] .
\end{aligned}
$$

Notice that we use different signs from the literature, due to our signature conventions: All perturbations enter with a positive coefficient proportional to $a^{2}$. One speaks of scalar, vector and tensor perturbations, respectively, when only the respective field perturbations are non-vanishing.

The fields $\phi, \psi, E, B, S_{a}, F_{a}, h_{a b}$ are not invariant under (infinitesimal) gauge transformations, that is, spacetime diffeomorphisms. In general, a metric changes under an infinitesimal diffeomorphism generated by a spacetime vector field $u^{\mu}$ by the corresponding Lie derivative

$$
\delta_{u} g_{\mu \nu}=\left[\frac{d}{d t}\left[\left(\varphi_{t}^{u}\right)^{*} g\right]_{\mu \nu}\right]_{t=0}=:\left[\mathcal{L}_{u} g\right]_{\mu \nu}=u^{\rho} g_{\mu \nu, \rho}+2 u_{,(\mu}^{\rho} g_{\nu) \rho}
$$


where $t \mapsto \varphi_{t}^{u}$ is the one parameter family of diffeomorphisms generated by the integral curves of the vector field $u$ [19]. If we take the order of $u$ to be the same as the order of the general perturbations, then we see that we can gauge away four of the ten perturbation fields. We define $u_{\mu}:=\eta_{\mu \nu} u^{\nu}$, that is $u_{0}=-u^{0}, u_{a}=u^{a}$ and $u:=\Delta^{-1} u_{, a}^{a}, u_{a}^{\perp}=u_{a}-\partial_{a} u$, where $\Delta^{-1}$ is the Green function associated to $\Delta$. We find explicitly

$$
\begin{aligned}
\delta_{u} g_{\mu \nu} & =a^{2}\left(2 \mathcal{H} u_{0} \eta_{\mu \nu}+2 u_{(\mu, \nu)}\right) \\
\delta_{u} g_{00} & =2 a^{2}\left(\frac{1}{a}(a u)^{\prime}\right) \\
\delta_{u} g_{0 a} & =a^{2}\left(\left[u_{0}+u^{\prime}\right]_{, a}+u_{a}^{\perp \prime}\right) \\
\delta_{u} g_{a b} & =2 a^{2}\left(-\mathcal{H} u_{0} \delta_{a b}+u_{, a b}+u_{(a, b)}^{\perp}\right) .
\end{aligned}
$$

Comparing with (A.30), we read off

$$
\begin{aligned}
\delta_{u} \phi & =\frac{1}{a}\left(a u_{0}\right)^{\prime} \\
\delta_{u} B & =u_{0}+u^{\prime} \\
\delta_{u} \psi & =-\mathcal{H} u_{0} \\
\delta_{u} E & =u \\
\delta_{u} S_{a} & =u_{a}^{\perp \prime} \\
\delta_{u} F_{a} & =u_{a}^{\perp} \\
\delta h_{a b} & =0 .
\end{aligned}
$$

From (A.33) we immediately see that a complete and linearly independent set of linear invariants under spacetime diffeomorphisms is given by

$$
\Phi=\phi-\frac{1}{a}\left[a\left(B-E^{\prime}\right)\right]^{\prime}, \quad \Psi=\psi+\mathcal{H}\left(B-E^{\prime}\right), \quad V_{a}=S_{a}-F_{a}^{\prime}, \quad h_{a b},
$$

which are six gauge invariant degrees of freedom. This follows from the fact that

$$
\delta_{u}\left(B-E^{\prime}\right)=u_{0}, \quad \delta_{u} E=u, \quad \delta_{u} F_{a}=u_{a}^{\perp} .
$$

A scalar field transforms under diffeomorphisms as

$$
\delta_{u} \zeta=u^{\mu} \zeta_{, \mu}
$$

which to linear order equals $u^{0} \bar{\zeta}^{\prime}=-\bar{\zeta}^{\prime} u_{0}$. Thus the linearly invariant scalar field perturbation is simply given by

$$
Z:=\delta \zeta+\bar{\zeta}^{\prime}\left(B-E^{\prime}\right)
$$

Just like the metric $g_{\mu \nu}$, any symmetric tensor such as the Einstein tensor $G_{\mu \nu}$ or the energy momentum tensor $T_{\mu \nu}$ transforms as in (A.31) under infinitesimal spacetime diffeomorphisms. Therefore, the Einstein equations with cosmological constant, $\Lambda$

$$
E_{\mu \nu}:=G_{\mu \nu}+\Lambda g_{\mu \nu}-\frac{\kappa}{2} T_{\mu \nu}=0
$$

are not written just in terms of the invariants (A.34). However, notice that for any symmetric tensor $t_{\mu \nu}$

$$
\begin{aligned}
\delta_{u} t_{\mu \nu} & =u^{0} \bar{t}_{\mu \nu}^{\prime}+2 u_{,(\mu}^{\rho} \bar{t}_{\nu) \rho} \\
& =u^{0} \bar{t}_{\mu \nu}^{\prime}+2 u_{,(\mu}^{0} \bar{t}_{\nu) 0}+2 \delta^{a b} u_{, a(\mu} \bar{t}_{\nu) b}+2 \delta^{a b} u_{a,(\mu}^{\perp} \bar{t}_{\nu) b} .
\end{aligned}
$$


Recalling (A.35) we see that the tensor

$$
\tilde{t}_{\mu \nu}:=t_{\mu \nu}+\left(B-E^{\prime}\right) \bar{t}_{\mu \nu}^{\prime}+2\left(B-E^{\prime}\right)_{,(\mu} \bar{t}_{\nu) 0}-2 \delta^{a b} E_{, a(\mu} \bar{t}_{\nu) b}-2 \delta^{a b} F_{a,(\mu} \bar{t}_{\nu) b}
$$

is gauge invariant. Now if we choose $t_{\mu \nu}=E_{\mu \nu}$ then $\bar{t}_{\mu \nu}=0$, since the FRW metric solves the Einstein equations. Therefore $E_{\mu \nu}=\tilde{E}_{\mu \nu}$ is gauge invariant, and in the second form it can be written just in terms of invariants when decomposing $\bar{E}_{\mu \nu}=\bar{G}_{\mu \nu}+\Lambda \bar{g}_{\mu \nu}-\kappa \bar{T}_{\mu \nu} / 2=0$. The same holds for the perturbations. Explicitly, for instance,

$$
\begin{aligned}
& \delta \tilde{G}_{00}=\delta G_{00}+\left(B-E^{\prime}\right) \bar{G}_{00}^{\prime}+2\left(B-E^{\prime}\right)^{\prime} \bar{G}_{00} \\
& \delta \tilde{G}_{0 a}=\delta G_{0 a}+\left[B-E^{\prime}\right]_{, a} \bar{G}_{00}-\frac{1}{3} \delta^{c d}\left(F_{a}+E_{, a}\right)^{\prime} \bar{G}_{c d} \\
& \delta \tilde{G}_{a b}=\delta G_{a b}+\left(B-E^{\prime}\right) \bar{G}_{a b}^{\prime}-\frac{2}{3} \delta^{c d}\left[F_{(a}+E_{,(a}\right]_{, b)} \bar{G}_{c d}
\end{aligned}
$$

where we used $\bar{G}_{00}=3 \mathcal{H}^{2}, \bar{G}_{0 a}=0, \bar{G}_{a b}=-\left(2 \mathcal{H}^{\prime}+\mathcal{H}^{2}\right) \delta_{a b}$. Equation (A.41) equals (A.40) with $G_{\mu \nu}$ replaced by $t_{\mu \nu}$, whenever $\bar{t}_{\mu \nu}$ is diagonal. Notice that the additional terms in (A.41) only contribute to scalar and vector perturbation contributions. Hence $\delta \tilde{G}_{\mu \nu}=\delta G_{\mu \nu}$ for tensor perturbations. We will see this explicitly.

Applying (A.40) to $t_{\mu \nu}=g_{\mu \nu}$ we find

$$
\delta \tilde{g}_{00}=2 a^{2} \Phi, \quad \delta \tilde{g}_{0 a}=a^{2} V_{a}, \quad \delta \tilde{g}_{a b}=2 a^{2} \Psi \delta_{a b}
$$

while, applying it to $t_{\mu \nu}=T_{\mu \nu}$, we find after some algebra

$$
\begin{aligned}
\delta \widetilde{T}_{00} & =\frac{1}{2 \lambda}\left(2 \bar{\zeta}^{\prime} Z^{\prime}+a^{2} v^{\prime}(\bar{\zeta}) Z-2 v(\bar{\zeta}) a^{2} \Phi\right) \\
\delta \widetilde{T}_{0 a} & =\frac{1}{\lambda}\left(\bar{\zeta}^{\prime} Z_{, a}+a^{2} \lambda \bar{p} V_{a}\right) \\
\delta \widetilde{T}_{a b} & =\frac{1}{\lambda}\left[\lambda \bar{p} a^{2} h_{a b}+2 \Psi \delta_{a b}+\frac{1}{2} \delta_{a b}\left(2\left(\bar{\zeta}^{\prime}\right)^{2} \Phi+2 \bar{\zeta}^{\prime} Z^{\prime}-a^{2} v^{\prime}(\bar{\zeta}) Z\right)\right] .
\end{aligned}
$$

The computation for the Einstein tensor itself is more complicated and we divide it into modes.

\section{A.5 Tensor perturbations}

For tensor perturbations we have $\delta g_{00}=\delta g_{0 a}=\delta^{c d} \delta g_{a c, d}=\delta^{c d} \delta g_{c d}=0$ and $\delta g_{a b}=a^{2} h_{a b}$. It immediately follows from (A.22) that $\delta R_{00}=\delta R_{0 a}=0$ and

$$
\delta R_{a b}=\left(\frac{1}{2 a^{2}} \delta g_{a b}^{\prime}\right)^{\prime}-2 \mathcal{H}\left(\frac{1}{2 a^{2}} \delta g_{a b}\right)^{\prime}+\frac{\mathcal{H}}{a^{2}} \delta g_{a b}^{\prime}-\frac{1}{2 a^{2}} \Delta \delta g_{a b}
$$

from which we immediately infer $\delta R=0$, so that $\delta\left(g_{a b} R\right)=\bar{R} \delta g_{a b}$. Using $\bar{R}=6\left(\mathcal{H}^{\prime}+\mathcal{H}^{2}\right) / a^{2}$, we find $\delta G_{00}=\delta G_{0 a}=0$ and for $\delta G_{a b}=\delta R_{a b}-\frac{1}{2} \bar{R} \delta g_{a b}$ with $\delta g_{a b}=a^{2} h_{a b}$ after some algebra

$$
\delta G_{a b}=\frac{1}{2}\left(h_{a b}^{\prime \prime}+2 \mathcal{H} h_{a b}^{\prime}-\Delta h_{a b}\right)-\left(2 \mathcal{H}^{\prime}+\mathcal{H}^{2}\right) h_{a b} .
$$

As already mentioned, $\delta G_{\mu \nu}=\delta \tilde{G}_{\mu \nu}$ for tensor perturbations.

The term in the second square bracket of (A.45) is not displayed in [9]. However, notice that there one perturbs the mixed components $G_{\nu}^{\mu}=g^{\mu \rho} G_{\rho \nu}$. Thus

$$
\delta G_{\nu}^{\mu}=-\bar{g}^{\mu \sigma} \delta g_{\sigma \lambda} \bar{g}^{\lambda \rho} \bar{G}_{\rho \nu}+\bar{g}^{\mu \rho} \delta G_{\rho \nu} .
$$


Hence $\delta G_{0}^{0}=\delta G_{a}^{0}=0$ and

$$
\delta G_{b}^{a}=-\frac{1}{a^{4}} \delta g_{a c} \bar{G}_{c b}+\frac{1}{a^{2}} \delta G_{a b}=\frac{1}{a^{2}}\left(-h_{a b} \delta^{c d} \frac{\bar{G}_{c d}}{3}+\delta G_{a b}\right)=\frac{1}{2 a^{2}}\left(h_{a b}^{\prime \prime}+2 \mathcal{H} h_{a b}^{\prime}-\Delta h_{a b}\right),
$$

where we used that $\delta^{c d} \bar{G}_{c d} / 3=-\left(2 \mathcal{H}^{\prime}+\mathcal{H}^{2}\right)$. This is the equation we find in [9]. The reason why we display here the twice covariant tensor components is that it is this form that one finds more directly in the Hamiltonian formulation.

\section{A.6 Vector perturbations}

In this case $\delta g_{00}=0, \delta g_{0 a}=a^{2} S_{a}, \delta g_{a b}=2 a^{2} F_{(a, b)}$. Since both $S_{a}, F_{a}$ are transversal, we have $\delta^{c d} \delta g_{0 c, d}=$ $\delta^{c d} \delta g_{c d}=0$, so that we immediately find from (A.22) that $\delta R_{00}=0$. The remaining equations in (A.22) simplify to

$$
\begin{aligned}
\delta R_{0 a} & =-\frac{1}{2} \Delta V_{a}+\left(\mathcal{H}^{\prime}+2 \mathcal{H}^{2}\right) S_{a} \\
\delta R_{a b} & =-\left(V_{(a, b)}^{\prime}+2 \mathcal{H} V_{(a, b)}\right)+2\left(\mathcal{H}^{\prime}+2 \mathcal{H}^{2}\right) F_{(a, b)}
\end{aligned}
$$

where we have used the linearly gauge invariant variable $V_{a}=S_{a}-F_{a}^{\prime}$. Next from (A.23) we immediately see that $\delta R=0$ so that $\delta\left(g_{\mu \nu} R\right)=\bar{R} \delta g_{\mu \nu}$. Hence $\delta G_{00}=0$ and

$$
\begin{aligned}
\delta G_{0 a} & =-\frac{1}{2} \Delta V_{a}+\left(\delta^{c d} \frac{\bar{G}_{c d}}{3}\right) S_{a} \\
\delta G_{a b} & =-\left(V_{(a, b)}^{\prime}+2 \mathcal{H} V_{(a, b)}\right)+2\left(\delta^{c d} \frac{\bar{G}_{c d}}{3}\right) F_{(a, b)}
\end{aligned}
$$

where $\delta^{c d} \bar{G}_{c d} / 3=-2 \mathcal{H}^{\prime}+\mathcal{H}^{2}$. Comparing with (A.41) we see that $\delta \tilde{G}_{00}=0$ and

$$
\begin{aligned}
\delta \tilde{G}_{0 a} & =-\frac{1}{2} \Delta V_{a}+\delta^{c d} \frac{\bar{G}_{c d}}{3} V_{a} \\
\delta \tilde{G}_{a b} & =-V_{(a, b)}^{\prime}-2 \mathcal{H} V_{(a, b)} .
\end{aligned}
$$

These are also the equations for the mixed components of the gauge invariant Einstein tensor that we find in [9].

\section{A.7 Scalar perturbations}

Now we have $\delta g_{00}=2 \phi a^{2}, \delta g_{0 a}=B_{, a} a^{2}, \delta g_{a b}=2 a^{2}\left(\psi \delta_{a b}+E_{, a b}\right)$. Hence $\delta^{c d} \delta g_{0 c, d}=a^{2} \Delta B, \delta^{c d} \delta g_{c d}=$ $2 a^{2}(3 \psi+\Delta E)$. Thus (A.22) simplifies to

$$
\begin{aligned}
& \delta R_{00}=-\Delta \Phi-3\left(\psi^{\prime \prime}+\mathcal{H}(\psi+\phi)^{\prime}\right) \\
& \delta R_{0 a}=-2\left[\Psi^{\prime}+\mathcal{H} \Phi\right]_{, a}+3 \mathcal{H}^{\prime}\left(B-E^{\prime}\right)_{, a}+\left(\mathcal{H}^{\prime}+2 \mathcal{H}^{2}\right) E_{, a}^{\prime} \\
& \delta R_{a b}=\left[-\Psi+\Phi+\left(\mathcal{H}^{\prime}+2 \mathcal{H}^{2}\right) E\right]_{, a b}+\left(-\Delta \Psi+\psi^{\prime \prime}+2\left(\mathcal{H}^{\prime}+2 \mathcal{H}^{2}\right)(\psi+\phi)+5 \mathcal{H} \psi^{\prime}+\mathcal{H} \phi^{\prime}\right) \delta_{a b},
\end{aligned}
$$

where we again used the gauge invariant variables $\Psi=\psi+\mathcal{H}\left(B-E^{\prime}\right)$ and $\Phi=\phi-\mathcal{H}\left(B-E^{\prime}\right)-\left(B-E^{\prime}\right)^{\prime}$. As an intermediate result we have

$$
\delta^{c d} \delta R_{c d}=\Delta\left[-\Psi+\Phi+\left(\mathcal{H}^{\prime}+2 \mathcal{H}^{2}\right) E\right]+3\left(-\Delta \Psi+\psi^{\prime \prime}+2\left(\mathcal{H}^{\prime}+2 \mathcal{H}^{2}\right)(\psi+\phi)+5 \mathcal{H} \psi^{\prime}+\mathcal{H} \phi^{\prime}\right) .
$$


Using (A.25) we find after some elaborate algebraic manipulations

$$
\begin{aligned}
\delta G_{00}= & 2\left(-\Delta \Psi+3 \mathcal{H} \psi^{\prime}\right) \\
\delta G_{0 a}= & -2\left[\Psi^{\prime}+\mathcal{H} \Phi\right]_{, a}-\bar{G}_{00}\left(B-E^{\prime}\right)_{, a}+\frac{1}{3} \delta^{c d} \bar{G}_{c d} E_{, a}^{\prime} \\
\delta G_{a b}= & {\left[-\Psi+\Phi-2\left(2 \mathcal{H}^{\prime}+\mathcal{H}^{2}\right) E\right]_{, a b} } \\
& +\left[\Delta(\Psi-\Phi)-2\left(\Psi^{\prime \prime}+\left(2 \mathcal{H}^{\prime}+\mathcal{H}^{2}\right)(\Psi+\Phi)+\mathcal{H}(2 \Psi+\Phi)^{\prime}\right)+2\left(\mathcal{H}^{\prime \prime}+\mathcal{H} \mathcal{H}^{\prime}\right)\left(B-E^{\prime}\right)\right] \delta_{a b}
\end{aligned}
$$

where we used $\bar{G}_{00}=3 \mathcal{H}^{2}, \delta^{c d} \bar{G}_{c d} / 3=-\left[2 \mathcal{H}^{\prime}+\mathcal{H}^{2}\right]$.

Hence, by (A.41), we get

$$
\begin{aligned}
\delta \tilde{G}_{00} & =\delta G_{00}+\bar{G}_{00}^{\prime}\left(B-E^{\prime}\right)+2 \bar{G}_{00}\left(B-E^{\prime}\right)^{\prime} \\
& =\delta G_{00}+6 \mathcal{H}\left[\mathcal{H}\left(B-E^{\prime}\right)\right]^{\prime} \\
& =2\left(-\Delta \Psi+3 \mathcal{H} \Psi^{\prime}\right) \\
\delta \tilde{G}_{0 a} & =\delta G_{0 a}+\bar{G}_{00}^{\prime}\left[B-E^{\prime}\right]_{, a}-\frac{1}{3} \delta^{c d} \bar{G}_{c d} E_{, a}^{\prime} \\
& =-2\left[\Psi^{\prime}+\mathcal{H} \Phi\right]_{, a} \\
\delta \tilde{G}_{a b} & =\delta G_{a b}+\left(B-E^{\prime}\right) \bar{G}_{a b}^{\prime}-\frac{2}{3} \delta^{c d} E_{, a b} \bar{G}_{c d} \\
& =\delta G_{a b}-2\left(B-E^{\prime}\right)\left(\mathcal{H}^{\prime \prime}+\mathcal{H}^{\prime} \mathcal{H}\right) \delta_{a b}+2\left(2 \mathcal{H}^{\prime}+2 \mathcal{H}^{2}\right) E_{, a b} \\
& =[-\Psi+\Phi]_{, a b}-\left[\Delta(\Phi-\Psi)+2\left(\Psi^{\prime \prime}+\left(2 \mathcal{H}^{\prime}+\mathcal{H}^{2}\right)(\Psi+\Phi)+\mathcal{H}(2 \Psi+\Phi)^{\prime}\right)\right] \delta_{a b} .
\end{aligned}
$$

The term $\left(2 \mathcal{H}^{\prime}+\mathcal{H}^{2}\right) \Psi$ disappears in $\delta \tilde{G}_{b}^{a}$ of [9], due to the variation of the additional metric contraction involved, as one can explicitly check.

\section{B Linear Perturbations following an Alternative Route}

In the main text we computed the linear perturbations of the general equations motion for our manifestly gauge-invariant configuration observables, the metric $Q_{j k}$ and the scalar field $\Xi$. That is, we used the secondorder temporal derivative form of the equations of motion, in which the canonical momenta were eliminated by using the Hamiltonian equations of motion. This is rather tedious, because one cannot use the special symmetries of the background when eliminating the momenta, but has to assume a general background. As shown in the appendix of our companion paper [1, to linear order one can get those equations also by perturbing the general Hamiltonian equations of motion and then eliminating the perturbed momenta by using the perturbed equations of motion. This is shorter because one can use the properties of the background at an earlier stage in the computation. On the other hand, the procedure followed in the main text quickly becomes more economic for higher than linear order.

In this section we will carry out this alternative derivation as a check of the result obtained in the main text.

\section{B.1 Gauge invariant FRW equations}

We will need the gauge invariant FRW equations in Hamiltonian form:

The metric takes the form $\bar{Q}_{j k}=A^{2} \delta_{j k}$, where $A$ is the observable scale factor. The corresponding extrinsic curvature is given by

$$
\bar{K}_{j k}=\frac{1}{2 \bar{N}}\left(\dot{\bar{Q}}_{j k}-\mathcal{L}_{\vec{N}} \bar{Q}_{j k}\right)=A \dot{A} \delta_{j k} .
$$

The momentum $\bar{P}^{j k}$ conjugate to $\bar{Q}_{j k}$ is given by

$$
\bar{P}^{j k}=\sqrt{\operatorname{det}(\bar{Q})}\left(\bar{Q}^{j m} \bar{Q}^{k n}-\bar{Q}^{j k} \bar{Q}^{m n}\right) \bar{K}_{m n}=-2 \dot{A} \delta^{j k}=: I \delta^{j k} .
$$


The background scalar fields $\bar{\Xi}, \bar{\Pi}$ are simply spatially homogeneous where $\bar{\Pi}$ is given by

$$
\bar{\Pi}=\sqrt{\operatorname{det}(\bar{Q})}\left(\dot{\bar{\Xi}}-\bar{N}^{j} \bar{\Xi}_{, j}\right)=A^{3} \dot{\bar{\Xi}}
$$

The symplectic potential is 8

$$
\Theta=\frac{1}{\kappa} \dot{\bar{Q}}_{j k} \bar{P}^{j k}+\frac{1}{\lambda} \dot{\Xi}_{0} \bar{\Pi}=\frac{1}{\kappa} \dot{A}(6 I A)+\frac{1}{\lambda} \dot{\Xi}_{0} \bar{\Pi}
$$

Thus, with

$$
J:=6 I A=-12 A \dot{A}, \quad I=\frac{J}{6 A}=-2 \dot{A},
$$

we see that the non-vanishing background Poisson brackets are

$$
\{J, A\}^{-}=\kappa, \quad\{\bar{\Pi}, \bar{\Xi}\}^{-}=\lambda .
$$

It follows that

$$
\bar{C}_{j}=-2 \bar{D}_{k} \bar{P}_{j}^{k}+\overline{\Pi D}_{j} \bar{\Xi}=0
$$

and

$$
\begin{aligned}
\kappa \bar{C}_{\text {geo }} & =\frac{2}{\sqrt{\operatorname{det}(\bar{Q})}} \bar{G}_{j k m n} \bar{P}^{j k} \bar{P}^{m n}-\sqrt{\operatorname{det}(Q)} R[\bar{Q}]+2 \Lambda \sqrt{\operatorname{det}(\bar{Q})} \\
& =-\frac{3}{2} A I^{2}+2 A^{3} \Lambda \\
& =-\frac{J^{2}}{24 A}+2 A^{3} \Lambda \\
\lambda C_{\text {matter }} & =\frac{1}{2 \lambda}\left[\frac{\bar{\Pi}^{2}}{\sqrt{\operatorname{det}(\bar{Q})}}+\sqrt{\operatorname{det}(\bar{Q})}\left(\bar{Q}^{j k} \bar{\Xi}_{, j} \bar{\Xi}_{, k}+v(\bar{\Xi})\right)\right] \\
& =\frac{1}{2 \lambda}\left(\frac{\bar{\Pi}^{2}}{A^{3}}+A^{3} v(\bar{\Xi})\right) \\
& =: \bar{\rho} A^{3} .
\end{aligned}
$$

Here we have introduced the background matter energy density $\bar{\rho}$. Notice that $\bar{H}=\bar{C}=\bar{C}_{\text {geo }}+\bar{C}_{\text {matter }}$, so that $\bar{C}$ is also the background Hamiltonian.

The Hamiltonian background equations of motion are

$$
\begin{aligned}
\dot{\Xi} & =\lambda \frac{\partial \bar{C}}{\partial \bar{\Pi}}=\frac{\bar{\Pi}}{A^{3}} \\
\dot{A} & =\kappa \frac{\partial \bar{C}}{\partial J}=-\frac{J}{12 A} \\
\dot{\bar{\Pi}} & =-\lambda \frac{\partial \bar{C}}{\partial \bar{\Xi}}=-\frac{1}{2} A^{3} v^{\prime}(\bar{\Xi}) \\
\dot{J} & =-\kappa \frac{\partial \bar{C}}{\partial A}=-\left[\left(\frac{J^{2}}{24 A^{2}}+6 A^{2} \Lambda\right)+\frac{3 \kappa}{2 \lambda}\left(-\frac{\bar{\Pi}^{2}}{A^{4}}+A^{2} v(\bar{\Xi})\right)\right]=:-\left[\left(\frac{J^{2}}{24 A^{2}}+6 A^{2} \Lambda\right)-3 A^{2} \bar{p}\right],
\end{aligned}
$$

where we have introduced the background matter pressure $\bar{p}$.

\footnotetext{
${ }^{8}$ This involves spatial averaging over the general symplectic structure, or, alternatively, we only use the integrand. The justification for this is that the corresponding equations of motion derived via the Poisson brackets correctly reproduce the connection between $\dot{A}, \dot{\bar{\Xi}}$ and the momentum conjugate to $A, \bar{\Xi}$ displayed in (B.2) and (B.3), respectively.
} 
The first two equations in (B.9) correctly reproduce the equations $\bar{\Pi}=A^{3} \dot{\bar{\Xi}}$ and $J=-12 A \dot{A}$ or $I=-2 \dot{A}$. Taking the second time derivatives of $A, \bar{\Xi}$ and using the last two equations in (B.9) , we find

$$
\begin{aligned}
\ddot{\Xi} & =-\frac{1}{2} v^{\prime}(\bar{\Xi})-3 \frac{\dot{A}}{A} \dot{\Xi} \\
3 \frac{\ddot{A}}{A} & =\Lambda-\frac{\kappa}{4}\left(\bar{\rho}+3 \bar{p}-\frac{\bar{\epsilon}}{A^{3}}\right) .
\end{aligned}
$$

Here we have used the conservation law that the total energy density $\bar{H}=\bar{C}=: \bar{\epsilon}>0$ is a constant of motion of the background Hamiltonian (it is not a constraint). The conservation equation $C=\bar{\epsilon}$ can be solved for $\dot{A}^{2}$ when using $J=-12 A \dot{A}$ and one finds

$$
3\left(\frac{\dot{A}}{A}\right)^{2}=\Lambda+\frac{\kappa}{2}\left(\bar{\rho}-\frac{\bar{\epsilon}}{A^{3}}\right),
$$

which displays $\bar{\rho}_{\text {dust }}=-\bar{\epsilon} / A^{3}$ as background dust energy density while $\bar{p}_{\text {dust }}=0$, since the dust is pressureless.

Equations (B.10) and (B.11) are the familiar FRW equations. However, they now describe the physical evolution with respect to the physical Hamiltonian $H$ of observable quantities, rather than the gauge transformations of non-observables as is the case in the usual formalism. This happens due to the deparametrisation through the dust whose only fingerprint is in the additional terms proportional to $\bar{\epsilon}$.

\section{B.2 Linear perturbations of the Hamiltonian equations of motion}

We start from the general Hamiltonian equations of motion for our physical degrees of freedom which we display once again below:

$$
\begin{aligned}
\dot{\Xi}= & \frac{N}{\sqrt{\operatorname{det}(Q)}} \Pi+\mathcal{L}_{\vec{N}} \Xi \\
\dot{\Pi}= & {\left[N \sqrt{\operatorname{det}(Q)} Q^{j k} \Xi_{, k}\right]_{, j}-\frac{N}{2} \sqrt{\operatorname{det}(Q)} v^{\prime}(\Xi)+\mathcal{L}_{\vec{N}} \Pi } \\
\dot{Q}_{j k}= & \frac{2 N}{\sqrt{\operatorname{det}(Q)}} G_{j k m n} P^{m n}+\left(\mathcal{L}_{\vec{N}} Q\right)_{j k} \\
\dot{P}^{j k}= & N\left[-\frac{Q_{m n}}{\sqrt{\operatorname{det} Q}}\left(2 P^{j m} P^{k n}-P^{j k} P^{m n}\right)+\frac{\kappa}{2} Q^{j k} C-\sqrt{\operatorname{det} Q} Q^{j k}\left(2 \Lambda+\frac{\kappa}{2 \lambda}\left(\Xi^{, m} \Xi_{, m}+v(\Xi)\right)\right)\right] \\
& +\sqrt{\operatorname{det} Q}\left[G^{-1}\right]^{j k m n}\left(\left(D_{m} D_{n} N\right)-N R_{m n}[Q]\right)+\frac{\kappa}{2 \lambda} N \sqrt{\operatorname{det} Q} \Xi^{, j} \Xi^{, k} \\
& -\frac{1}{2} H Q^{j m} Q^{k n} N_{m} N_{n}+\left(\mathcal{L}_{\vec{N}} P\right)^{j k}
\end{aligned}
$$

with $N=\sqrt{1+Q^{j k} N_{j} N_{k}}, N_{j}=-C_{j} / H, H=\sqrt{C^{2}-Q^{j k} C_{j} C_{k}}$. Notice that in our convention $C=C_{\text {geo }}+$ $C_{\text {matter }}$ and $C_{\text {geo }}$ contains the cosmological constant term. The bimetric $G_{j k m n}=Q_{j(m} Q_{n) k}-1 / 2 Q_{j k} Q_{m n}$ has the inverse $\left(G^{-1}\right)^{j k m n}=\left[Q_{j(m} Q_{n) k}-Q_{j k} Q_{m n}\right]$, so that $G_{j k p q}\left(G^{-1}\right)^{p q m n}=\delta_{(j}^{m} \delta_{k)}^{n}$. In what follows, we again adopt the notation of the main text and denote background quantities by an overbar, while perturbations are denoted by the symbol $\delta$. For instance, $\delta Q_{j k}=Q_{j k}-\bar{Q}_{j k}$.

In perturbing equations (B.12) around the FRW background we make first the following observations which will drastically simplify the subsequent analysis:

1. In linear order the perturbation of the lapse vanishes

$$
\delta N=\frac{1}{\bar{N}}\left(\bar{Q}^{j k} \bar{C}_{j} \delta C_{k}+\frac{1}{2} \delta Q^{j k} \bar{C}_{j} \bar{C}_{k}\right)=0,
$$

since $C_{j}=-2 D_{k} P_{j}^{k}+\Pi D_{j} \Xi$. Hence $\bar{C}_{j}=0$ due to spatial homogeneity (in dust space $\mathcal{S}$ ). 
2. The same holds for the second covariant derivatives of the lapse

$$
\delta D_{j} D_{k} N=\delta\left(\partial_{j} \partial_{k} N-\Gamma_{j k}^{m} \partial_{m} N\right)=\partial_{j} \partial_{k} \delta N-\bar{\Gamma}_{j k}^{m} \partial_{m} \delta N+\delta \Gamma_{j k}^{m} \partial_{m} \bar{N}=0,
$$

since both $\bar{\Gamma}_{j k}^{m}=0, D_{m} \bar{N}=0$ due to spatial homogeneity.

3. The terms quadratic in spatial derivatives of the scalar field have vanishing variation because at least one of the spatial derivatives is not varied and then vanishes.

4. In the variation of the Ricci curvature terms only the term involving the variation of the Ricci curvature $R_{j k}$, which is linear in the Christoffel symbol, survives.

5. As shown in the appendix of [1], the linear perturbations of the conserved quantities $H(\sigma), C_{j}(\sigma)$ and thus $N_{j}=-C_{j} / H$ are conserved with respect to the linear equations of motion which are generated by the second order term of the perturbation of the physical Hamiltonian $\mathbf{H}$.

It follows that we can set everywhere $N=\bar{N}=1$. Notice however, that, while $\delta N \equiv 0, \bar{N}_{j} \equiv 0$, we have $\delta N_{j} \neq 0$.

With these preparations out of the way, we can now perturb (B.12). In order to keep the formulae simple at intermediate steps we define $\delta Q_{j k}=L \delta_{j k}, \delta P^{j k}=I \delta^{j k}$. Later we will substitute $L=A^{2}, I=-2 \dot{A}$. Remember that $P^{j k}, \Pi$ are densities of weight one, so that $\left(N^{j}=Q^{j k} N_{k}\right)$

$$
\mathcal{L}_{\vec{N}} P^{j k}=\partial_{m}\left(N^{m} P^{j k}\right)-2 N_{, m}^{(j} P^{k) m}, \quad \mathcal{L}_{\vec{N}} \Pi=\partial_{m}\left(N^{m} \Pi\right) .
$$

Since $\bar{N}_{j}=0$, we have

$$
\begin{aligned}
\delta \mathcal{L}_{\vec{N}} Q_{j k} & =\mathcal{L}_{\delta \vec{N}} \bar{Q}_{j k}=2 \delta N_{(j, k)} \\
\delta \mathcal{L}_{\vec{N}} \Xi & =0 \\
\delta \mathcal{L}_{\vec{N}} P^{j k} & =I\left(\left[\delta N^{m}\right]_{, m} \delta^{j k}-2\left[\delta N^{(j}\right]_{, m} \delta^{k) m}\right)=\frac{I}{L}\left(\delta^{m n} \delta^{j k}-2\left(\delta^{m(j} \delta^{k) n}\right)\left[\delta N_{m}\right]_{, n}\right. \\
& =-\frac{2 I}{L} \bar{G}_{j k m n}\left[\delta N_{m}\right]_{, n} \\
\delta \mathcal{L}_{\vec{N}} \Pi & =\partial_{m}\left(\delta N^{m} \bar{\Pi}\right)=\frac{\bar{\Pi}}{L}\left[\delta N_{m}\right]_{, m} .
\end{aligned}
$$

Here we have introduced the flat bimetric $\bar{G}_{j k m n}=\delta_{j(m} \delta_{n) k}-1 / 2 \delta_{j k} \delta_{m n}$ with inverse $\bar{G}_{j k m n}^{-1}=\delta_{j(m} \delta_{n) k}-$ $\delta_{j k} \delta_{m n}$, so that $\bar{G}_{j k p q} \bar{G}_{p q m n}=\delta_{j(m} \delta_{n) k}$. Also we will use Einstein summation convention in what follows, irrespective of index position on Kronecker $\delta$ 's, that is, we define $\bar{G}^{j k m n}:=\bar{G}_{j k m n}$ and $\left[\bar{G}^{-1}\right]^{j k m n}:=\left[\bar{G}^{-1}\right]_{j k m n}$, as well as $\delta^{j k}=\delta_{j k}$. However, notice that our fundamental perturbation quantities are $Q_{j k}, P^{j k}, N_{j}$ with that index position, and one has to take care of the additional metric contractions involved when the index is a priori not in that position. For instance,

$$
\delta Q^{j k}=-\left[\bar{Q}^{j m} \bar{Q}^{n k}\right] \delta Q_{m n}=-\frac{1}{L^{2}} \delta Q_{j k} .
$$

We also often need

$$
\begin{aligned}
\delta \sqrt{\operatorname{det}(Q)} & =\left[\frac{1}{2} \sqrt{\operatorname{det}(\bar{Q})} \bar{Q}^{m n}\right] \delta Q_{m n}=\frac{\sqrt{L}}{2} \delta Q_{m m} \\
\delta \frac{1}{\sqrt{\operatorname{det}(Q)}} & =-\frac{1}{L^{5 / 2}} \delta Q_{j j} .
\end{aligned}
$$


Then we find after straightforward, but tedious calculations (simply using the product rule for linear variations all the time)

$$
\begin{aligned}
\delta \dot{\Xi}= & \frac{\delta \Pi}{L^{3 / 2}}-\frac{\bar{\Pi}}{2 L^{5 / 2}} \delta Q_{m m} \\
\delta \dot{\Pi}= & \frac{1}{2}\left(-\frac{L^{1 / 2}}{2} v^{\prime}(\bar{\Xi}) \delta Q_{m m}-L^{3 / 2} v^{\prime \prime}(\bar{\Xi}) \delta \Xi+2 L^{1 / 2} \Delta \delta \Xi\right)+\frac{\bar{\Pi}_{L}}{L}\left[\delta N_{m}\right]_{, m} \\
\delta \dot{Q}_{j k}= & \frac{2}{L^{1 / 2}} \bar{G}_{j k m n}\left(L \delta P^{m n}+\frac{I}{2} \delta Q_{m n}\right)+2\left[\delta N_{(j}\right]_{, k)} \\
\delta \dot{P}^{j k}= & -\frac{2 I}{L} \bar{G}_{j k m n}\left[\delta N_{(m}\right]_{, n)}-\frac{I}{L^{1 / 2}} \bar{G}_{j k m n} \delta P^{m n}-\frac{1}{L^{1 / 2}} \bar{G}_{j k m n} \delta R_{m n} \\
& +\frac{\delta_{j k}}{L^{1 / 2}}\left[\frac{3}{8} \frac{I^{2}}{L}-\frac{\Lambda}{2}-\frac{\kappa}{4} \bar{\rho}\right] \delta Q_{m m}+\frac{1}{L^{1 / 2}}\left[-\frac{5}{4} \frac{I^{2}}{L}+\Lambda-\frac{\kappa}{2} \bar{p}\right] \delta Q_{j k} \\
& +\delta_{j k} \frac{\kappa}{4 \lambda}\left(2 \frac{\bar{\Pi}}{L^{5 / 2}} \delta \Pi-L^{1 / 2} v^{\prime}(\bar{\Xi}) \delta \Xi\right) .
\end{aligned}
$$

Here $\Delta=\delta^{m n} \partial_{m} \partial_{n}$ is the flat Laplacian and all derivatives appearing are flat derivatives. As in the previous subsection, we used $\bar{\rho}=\left[\bar{\Pi}^{2} / L^{3}+v(\bar{\Xi})\right] /(2 \lambda)$ and $\bar{p}=\left[\bar{\Pi}^{2} / L^{3}-v(\bar{\Xi})\right] /(2 \lambda)$. The variation of the Ricci tensor is given explicitly by

$$
\delta R_{j k}=\frac{1}{2 L}\left(2 \delta Q_{m(j, k) m}-\Delta \delta Q_{j k}-\delta Q_{m m, j k}\right) .
$$

\section{B.3 Second time derivative form of the perturbed equations of motion}

The explicit inversion of the first and third relation in (B.19) for the perturbed momenta in terms of the velocities yields

$$
\begin{aligned}
\delta \Pi & =L^{3 / 2} \delta \dot{\Xi}+\frac{\bar{\Pi}}{2 L} \delta Q_{m m} \\
\delta P^{j k} & =\frac{1}{2 L^{1 / 2}}\left[\bar{G}^{-1}\right]_{j k m n}\left(\delta \dot{Q}_{m n}-2\left[\delta N_{(m}\right]_{, n)}\right)-\frac{I}{2 L} \delta Q_{j k} .
\end{aligned}
$$

Taking the second derivative of the first and third relation in (B.19), we substitute for $\delta \dot{\Pi}, \delta \dot{P}^{j k}$, using the second and fourth relation in (B.19), respectively. Afterwards we substitute for $\delta \Pi, \delta P^{j k}$, using (B.21). In doing that, one has to remember that $d / d \tau \delta N_{j}=0$, i.e. $\delta N_{j}$ is a constant of motion.

We find again after some tedious algebra?

$$
\begin{aligned}
\delta \ddot{\Xi} & =-\frac{3 \dot{L}}{2 L} \delta \dot{\Xi}-\frac{1}{2} v^{\prime \prime} \delta \Xi+\frac{1}{L} \Delta \delta \Xi+\left[\frac{\dot{L} \bar{\Pi}}{2 L^{7 / 2}}-\frac{\dot{\bar{\Pi}}}{2 L^{5 / 2}}-\frac{v^{\prime}(\bar{\Xi})}{4 L}\right] \delta Q_{m m}-\frac{\bar{\Pi}}{2 L^{5 / 2}} \delta \dot{Q}_{m m}+\frac{\bar{\Pi}}{L^{5 / 2}}\left[\delta N_{m}\right]_{, m} \\
\delta \ddot{Q}_{j k} & =\frac{\dot{L}}{2 L}\left(\delta \dot{Q}_{j k}-2\left[\delta N_{(j, j)}\right)+\frac{1}{L^{1 / 2}} \bar{G}_{j k m n}\left(\dot{I} \delta Q_{m n}+I \delta \dot{Q}_{m n}-I \frac{\dot{L}}{L} \delta Q_{m n}\right)+2 L^{1 / 2} \bar{G}_{j k m n} \delta \dot{P}^{m n} .\right.
\end{aligned}
$$

It is a good check to verify that the dimensionalities of the various terms match: in our convention, the spatial coordinates $\sigma^{j}$ are chosen to be dimensionfree, while $\tau$ has dimension of length. Thus $L$ has dimension $\mathrm{cm}^{2}$ and $A$ has dimension $\mathrm{cm}^{1}$. The scalar field $\Xi$ is dimensionfree, hence $\Pi \propto A^{3} \dot{\Xi}$ has dimension $\mathrm{cm}^{2}$. The potential term $v(\bar{\Xi})$ has the same dimension as $\Pi^{2} / L^{3}$ which is $\mathrm{cm}^{-2}$. Likewise, $Q_{j k}$ has dimension $\mathrm{cm}^{2}$ while $K_{j k} \propto \dot{Q}_{j k} / N$ has dimension $\mathrm{cm}^{1}$ so that $P^{j k} \propto \sqrt{\operatorname{det}(Q)} Q^{j k} Q^{m n} K_{m n}$ is actually dimensionless, just like $I=-2 \dot{A}$.

\footnotetext{
${ }^{9}$ One should really write $d^{2} /(d \tau)^{2} \delta Q_{j k}$, rather than $\delta \ddot{Q}_{j k}$ etc. However, these two quantities are numerically identical.
} 
In the last line of (B.22) we still must insert the last relation of (B.19). Since there are various bimetric contractions involved, we notice the identities

$$
\bar{G}_{j k p q} \bar{G}_{p q m n}=\delta_{j(m} \delta_{n) k}-\frac{1}{4} \delta_{j k} \delta_{m n}, G_{j k m n} \delta_{m n}=-\frac{1}{2} \delta_{j k} .
$$

The calculation is very tedious but the result is rather simple. We directly substitute $L=A^{2}$ and $I=-2 \dot{A}$ and find

$$
\begin{aligned}
\delta \ddot{Q}_{j k}= & \frac{\dot{A}}{A} \delta \dot{Q}_{j k}+2 \frac{\dot{A}}{A}\left[\delta N_{(j}\right]_{k)}+2\left[-\left(\frac{\dot{A}}{A}\right)^{2}-\frac{\ddot{A}}{A}+\left(\Lambda-\frac{\kappa}{2} \bar{p}\right)\right] \delta Q_{j k}-2 \delta R_{j k} \\
& +\delta_{j k}\left(\left[\frac{\ddot{A}}{A}+\frac{1}{2}\left(\frac{\dot{A}}{A}\right)^{2}-\frac{1}{2} \Lambda+\frac{\kappa}{4} \bar{p}\right] \delta Q_{m m}+\frac{1}{2} \delta R_{m m}-\frac{\kappa}{2 \lambda} A^{2}\left(\dot{\bar{\Xi}} \delta \dot{\Xi}-\frac{1}{2} v^{\prime}(\bar{\Xi}) \delta \Xi\right)\right) .
\end{aligned}
$$

We can simplify this expression further by making use of the background equations (B.10) and (B.11) which imply

$$
2 \frac{\ddot{A}}{A}+\left(\frac{\dot{A}}{A}\right)^{2}=\Lambda-\frac{\kappa}{2} \bar{p} .
$$

Therefore the $\delta Q_{m m}$ term in (B.24) vanishes and the $\delta Q_{j k}$ term simplifies, leading to

$$
\delta \ddot{Q}_{j k}=\frac{\dot{A}}{A} \delta \dot{Q}_{j k}+2 \frac{\dot{A}}{A}\left[\delta N_{(j}\right]_{k)}+2 \frac{\ddot{A}}{A} \delta Q_{j k}-2 \delta R_{j k}+\delta_{j k}\left(\frac{1}{2} \delta R_{m m}-\frac{\kappa}{2 \lambda} A^{2}\left(\dot{\bar{\Xi}} \delta \dot{\Xi}-\frac{1}{2} v^{\prime}(\bar{\Xi}) \delta \Xi\right)\right)(. \mathrm{B} .2
$$

Equations (B.22) and (B.25) are precisely equations (2.17) and (2.21), derived by the more general formalism of section 2,

\section{References}

[1] K. Giesel, S. Hofmann, T. Thiemann, O. Winkler, Manifestly gauge-invariant general relativistic perturbation theory: I. Foundations. arXiv:0711.0115 [gr-qc]]

[2] C. Rovelli. What is observable in classical and quantum gravity? Class. Quantum Grav. 8 (1991), 297-316.

C. Rovelli. Quantum reference systems. Class. Quantum Grav. 8 (1991), 317-332.

C. Rovelli. Time in quantum gravity: physics beyond the Schrodinger regime. Phys. Rev. D43 (1991), 442-456.

C. Rovelli. Quantum mechanics without time: a model. Phys. Rev. D42 (1990), 2638-2646.

[3] B. Dittrich. Partial and complete observables for Hamiltonian constrained systems. gr-qc/0411013

B. Dittrich. Partial and complete observables for canonical general relativity. Class. Quant. Grav. 23 (2006),6155-6184. gr-qc/0507106

[4] J. Brown and K. Kuchař. Dust as a standard of space and time in canonical quantum gravity. Phys. Rev. D51 (1995), 5600-5629. gr-qc/9409001

[5] T. Thiemann. Solving the problem of time in general relativity and cosmology with phantoms and k-essence. astro-ph/0607380

[6] E. M. Lifshitz, J. Phys. USSR 10, 116 (1946).

[7] J. M. Bardeen, Gauge-invariant cosmological perturbations, Phys. Rev. D22, 1882 (1980).

[8] H. Kodama and M. Sasaki, Cosmological perturbation theory, Prog. Theor. Phys. Suppl. 78, 1 (1984).

[9] V. Mukhanov, H. Feldman and R. Brandenberger. Theory of cosmological perturbations. Part 1. Classical perturbations. Part 2. Quantum theory of perturbations. Part 3. Extensions. Phys. Rept. 215 (1992) 203-333.

[10] H. Noh and J. Hwang, Second-order perturbations of the Friedmann world model, Phys. Rev. D69, 104011 (2004). 
[11] K. Nakamura, Gauge invariant variables in two-parameter nonlinear perturbations, Progr. Theor. Phys. 110, 723 (2003).

[12] K. Nakamura, Second-order gauge invariant perturbation theory, Progr. Theor. Phys. 113, 723 (2005).

[13] K. Nakamura, Second-order gauge invariant cosmological perturbation theory, Progr. Theor. Phys. 117, 723 (2007).

[14] D. Langlois and F. Vernizzi, Evolution of nonlinear cosmological perturbations, Phys. Rev. Lett. 95, 091303 (2005).

[15] N. Bartolo, S. Matarrese and A. Riotto, The full second-order radiation transfer function for large-scale CMB anisotropies, JCAP 05, 010 (2006).

[16] G. F. R. Ellis and M. Bruni, Covariant and gauge-invariant approach to cosmological density fluctuations, Phys. Rev. D 40, 1804 (1989).

[17] M. Bruni, P. K. S. Dunsby and G. F. R. Ellis, Cosmological perturbations and the physical meaning of gauge-invariant variables, Astrophys. J. 395, 34 (1992).

[18] K. Giesel, S. Hofmann, T. Thiemann, O. Winkler, Manifestly gauge-invariant general relativistic perturbation theory: III. FRW background and second order. In Preparation.

[19] R. M. Wald. General Relativity, (The University of Chicago Press, Chicago, 1989).

[20] J.-A. Garcia and J. Pons. Rigid and gauge Noether symmetries for constrained systems. Int. J. Mod. Phys. A15 (2000) 4681. hep-th/9908151]

J.-A. Garcia and J. Pons. Lagrangian Noether symmetries as canonical transformations. Int. J. Mod. Phys. A16 (2001) 3897. hep-th/0012094

J. Pons. Generally covariant theories: the Noether obstruction for realising certain spacetime diffeomorphisms in phase space. Class.Quant.Grav, 20, 3279 (2003).

J. Pons, D. Salisbury and L. Shepley. Gauge transformations in the Lagrangian and Hamiltonian formalisms of generally covariant theories. Phys. Rev. D55 (1997) 658. [gr-qc/9612037]

J. Lee and R. Wald. Local symmetries and constraints. J. Math. Phys. 31 (1990) 725

[21] T. Thiemann. Reduced phase space quantization and Dirac observables. Class. Quant. Grav. 23 (2006), 1163-1180. gr-qc/0411031

[22] K. Giesel and T. Thiemann. Algebraic quantum gravity (AQG) IV. Reduced Phase Space Quantisation of Loop Quantum Gravity. arXiv:0711.0119 [gr-qc]]

[23] P. D. Death, On the existence of perturbed RobertsonWalker universes, Ann. Phys. N.Y. 98, 237 (1976).

L. Bruna and J. Girbau, Linearization stability of the Einstein equation for Robertson-Walker models. I, J. Math. Ph. 40, 5117 (1999).

L. Bruna and J. Girbau, Linearization stability of the Einstein equation for Robertson-Walker models. II, J. Math. Ph. 40, 5131 (1999). 